\title{
THE CASE FOR DIFFERENTIAL GEOMETRY IN THE CONTROL OF SINGLE AND COUPLED PDES: THE STRUCTURAL ACOUSTIC CHAMBER
}

R. GULLIVER* ${ }^{*}$, I. LASIECKA ${ }^{\dagger}$, W. LITTMAN*, AND R. TRIGGIANI ${ }^{\dagger}$

\section{Contents}

Part I: The need for differential geometry in the control of PDEs. Its main features . . . . . . . . . . . . . . .

0 Informal overview of some control theory concepts and terminology . . . . . . . . . . . . . . 4

1 Introduction: Aim and scope of the present paper. Qualitative statement of results. Two approaches . . . . . . . . . . 11

1.1 Linear single PDEs, or coupled systems of PDEs, with variable coefficients and/or defined on curved

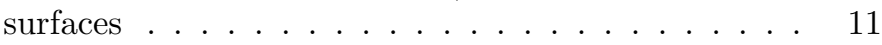

1.2 Two geometric approaches . . . . . . . . . . . 12

1.3 Summary of main features of differential geometric methods in the control of PDEs . . . . . . . . . . . 13

1.4 Geometric methods and control of PDEs: A two-way interaction .............. . . 14

1.5 Systems of coupled PDEs. Nonlinear problems . . . 15

2 A benchmark PDE-control problem advocating a broad use of differential geometry: The structural acoustic problem . . 16

2.1 Various structural acoustics models with constant coefficients and with flat flexible wall . . . . . . . . 17

2.2 More realistic models: Variable coefficients and curved walls . . . . . . . . . . . . . . . . 19

2.3 Conclusion ................ . 20

Part II: Riemannian geometric assumptions. Overview of results 20

3 Basic geometric assumption for the energy method approach in the Riemannian metric . . . . . . . . . . . . . . 22

3.1 Strictly convex functions and coercive fields in the Riemannian metric for hyperbolic and non-hyperbolic (Petrowski)-problems . . . . . . . . . . . 22

3.2 Use of assumption (H.1). General statement of Carleman estimates and overview . . . . . . . . . . 24

*School of Mathematics, Vincent Hall, University of Minnesota, Minneapolis, MN 55455

${ }^{\dagger}$ Department of Mathematics, Kerchof Hall, University of Virginia, Charlottesville, VA 22904. Research partially supported by the National Science Foundation under Grant DMS-0104305 and by the Army Research Office under Grant DAAD19-02-1-0179. 
4 Survey of geometric results and methods for the construction of strictly convex functions and coercive fields . . . . . . . 31

4.1 The need for a geometrical perspective . . . . . . . . 31

4.1.1 Euclidean Space . . . . . . . . . . 32

4.1.2 The Hessian Comparison Theorem . . . . . 32

4.2 Functions with convex level sets . . . . . . . . 33

4.3 The Matrix Riccati Equation . . . . . . . . . . 34

4.4 Matrix Riccati Comparison Theorem . . . . . . . . 35

4.5 Nonsmooth distance function . . . . . . . . . . 37

4.6 Construction of a strictly convex function in the 2 -d case via curvature flows . . . . . . . . . . 38

5 A Riemannian geometric approach for exact controllability of second-order hyperbolic equations. A "view from the boundary" which verifies the geometric optics condition . . 5.1 Introduction. Counterexamples. Hypotheses. Main exact controllability statement . . . . . . . . . . 39

5.2 Chord uniqueness and nondegeneracy . . . . . . 43

5.3 Criteria for chord uniqueness and non-degeneracy . . 44

5.4 Examples ................. . . 45

5.5 Sketch of proof of Theorem 5.1.1: Verification of the geometric optics conditions . . . . . . . . . . 47

Part III: Single PDE equations: Carleman estimates. Exact controllability. Uniform stabilization . . . . . . . . .

6 Second-order hyperbolic equations on a Riemannian manifold: Energy methods in the Riemannian metric yielding Carleman and control estimates with lower-order terms . . 6.1 Model. Euclidean versus Riemannian domain . . . . 6.2 Strictly convex functions and coercive fields in the Riemannian metric . . . . . . . . . . . . . 49

6.3 A second-order hyperbolic equation with variable coefficients defined on a Euclidean domain . . . . . . . 50

6.4 Carleman estimates for problem (6.1.1), (6.1.2) with lower-order terms . . . . . . . . . . . 51

6.5 Control-theoretic results for (6.1.1)-(6.1.3) . . . . . 54

6.6 Lower-level energy inequality: stabilization inequality with Dirichlet-boundary feedback in $\boldsymbol{L}_{\mathbf{2}}(\boldsymbol{\Sigma})$. . 59

6.7 Geometric optics and strictly convex functions . . . 61

7 Second-order hyperbolic equations: Energy method in the Riemannian metric yielding Carleman and control estimates with no lower-order terms. Purely Neumann B.C. Global unique continuation . . . . . . . . . . . .

8 Schrödinger equations on a Riemannian manifold: Energy methods in the Riemannian metric yielding Carleman/control estimates with lower-order terms . . . . . . . . . . 
9 Schrödinger equations: Energy method yielding Carleman/ control estimates with no lower-order terms. Purely Neumann B.C. Global unique continuation . . . . . . . . . . . .

10 Plate equations on the Riemannian manifold: Energy methods in the Riemannian metric yielding Carleman/control estimates with lower-order terms . . . . . . . . . .

Part IV: A dynamic shell model . . . . . . . . . . . . . . . . . .

11 Uniform stabilization of a shallow shell model with nonlinear boundary feedbacks. Critical marriage between Riemannian geometry (continuous observability inequality) and microlocal analysis (boundary trace estimates) . . . . . . . .

11.1 A differential geometric model for a dynamic shallow shell's model in nonlinear, dissipative, feedback form $\quad 89$

11.2 Main results: Well-posedness and uniform stabilization 91

11.3 Preliminary results . . . . . . . . . . . 95

11.4 First step of the proof: Local reduction to a Euclidean (flat) coordinate system . . . . . . . . 97

11.5 Trace regularity for elastic waves ( $W$-component) . . 98

11.6 Trace regularity for normal component $\boldsymbol{w}$. . . . . 100

11.7 Stabilizability estimate and completion of the proof of Theorem 11.2.2 . . . . . . . . . . . . 100

Part I: The need for differential geometry in the control of PDEs. Its main features.

Foreword. In line with the title of the IMA Summer Program-Geometric Methods in Inverse Problems and PDE Control - the aim of the present article may be summarized as follows: we intend to provide a relatively updated survey (subject to space limitations) of results on exact boundary controllability and uniform boundary stabilization of certain general classes of single Partial Differential Equations as well as of classes of systems of coupled PDEs (in dimension strictly greater than one), that have become available in recent years through novel approaches based on differential (Riemannian) geometric methods.

Thus, this is not a survey on all relevant methods that have been introduced in the control of PDEs (in dimension greater than one). Rather, this is a review article deliberately restricted and confined to approaches that make explicit and intense use of differential (Riemannian) geometric methods. Accordingly, some cornerstone approaches available in the literature of control of PDEs are in the background rather than on the forefront of this article. They include (but are not necessarily limited to):

(i) The geometric optics approach in an Euclidean domain, which is restricted, however, to hyperbolic dynamics. It was initiated by W. Littman [Lit.1] for hyperbolic systems controlled on the full boundary, and by J. Ralston [Ra.1] in the study of 'Gaussian beams,' and later carried out to a high level of generality by C. Bardos, J. Lebeau, and J. Rauch [B-L-R.1] for general second-order hyperbolic equations. This approach rests on an almost necessary and sufficient geometric 
optics assumption, which, however, is not readily checkable. It requires $C^{\infty}$ smoothness of both the Euclidean domain and the coefficients. Later, smoothness was greatly reduced to $C^{3}$-domains and $C^{2}$-coefficients in Burq [Bu.1], but only for the wave equation with Dirichlet-control, by using $H$-measures. An adaptation of this method to the pure Schrödinger equation is given in Lebeau [Le.2], under the same geometric optics condition.

(ii) The approach of D. Tataru [Ta.1-3] this time for general evolution equations (to include the single PDEs explicitly treated in the present article), which is based on pseudo-differential calculus (see his review article [Ta.8] on unique continuation on this same volume) to obtain first, as an intermediate step, Carlemantype estimates, under a not-so-easily checkable pseudo-convexity assumption, and next the sought-after control-theoretic inequalities.

The computational Riemannian geometric energy method approach to be treated in the present article shares, in common with the pseudo-differential approach of Tataru, the important feature that it applies to various single PDE equations, be they hyperbolic or not, including Petrowski-type PDEs (and other non-hyperbolic PDEs are expected to be included in the feature). As such, the computational Riemannian geometric method serves as a unifying tool applicable also to the study of systems of PDEs which couple single PDEs dynamics of various type, hyperbolic or not, of interest in applications. A canonical motivating illustration is the structural acoustic chamber of Section 2.

Much of the article - Parts I through III - is focused on general linear PDEs, while we refer to Section 1.5 below for information and insight on the corresponding nonlinear classes. However, Part IV considers the stabilization problem of the linear (coupled system of two hyperbolic-like PDEs known as) shallow shell, subject to suitable nonlinear dissipative terms. We quote from the IMA Workshop: "The IMA workshop goals are to bring together geometers with researchers in inverse problems and control of PDEs to facilitate exchange of ideas and encourage collaboration; to make tools of differential geometry known to those working in inverse problems and control, and to open new areas of research in geometry." In this spirit, we open up this paper with an introductory qualitative treatment, written for the benefit of those in areas other than control of PDEs. The expert in control of PDE may skip this introductory part and move directly to the variable coefficient case (Section 1).

0. Informal overview of some control theory concepts and terminology.

Exact boundary controllability and equivalent continuous observability inequality. Qualitatively, by exact boundary controllability of an evolution equation we mean the property of steering or transferring any initial condition at time $t=0$ to any target state at time $T$ (in suitable function spaces) by means of a non-homogeneous boundary function, called boundary control (in a suitable function space) acting on (possibly, a portion of) the boundary of the open bounded domain $\Omega$ in which the evolution is defined.

The optimal relationship between the function space that defines the class of boundary controls and the function space defining the correspond- 
ing solution (state) at $t=T$ is very important in itself, and critical in the area of optimal control, which justifies the interest in the property of exact boundary controllability in the first place [L-T.11]. Other questions such as: (i) "minimal" universal time of transfer (independent of the pair of initial and final state); (ii) "minimal" portion of the boundary required by the boundary control to perform any such transfer, are also of interest, though at a far lower order of priority in applications to optimal control theory.

Here we shall be concerned only with time reversible single or coupled evolution equations (second-order hyperbolic equations, Petrowski-systems such as Schrödinger equations and plate-like equations (hyperbolic or not), system of elasticity, shells, etc.). [An exception is the thermoelastic plate of Section 2.] Accordingly, w.l.o.g. we may take the initial condition to be the origin. We then seek to hit any target state (in a suitable function space) at time $T$ (universal) by means of a suitable class of boundary controls. This says, mathematically, that the 'control $\rightarrow$ solution map' $L_{T}$ (which may be either continuous, or else unbounded but closed)

$$
\begin{gathered}
L_{T}: \text { from the space of boundary controls be surjective (onto) } \\
\longrightarrow \text { state space of solutions at } t=T .
\end{gathered}
$$

The standard mathematical property (0.1) is re-labelled, in the control theory jargon, as exact boundary controllability at $T$. By a standard result in Functional Analysis [T-L.1, p. 235], the surjectivity property (0.1) is then equivalent to the property that the adjoint operator $L_{T}^{*}$ is bounded below: there exists a constant $c_{T}>0$ such that

$$
\left\|L_{T}^{*} z\right\| \geq c_{T}\|z\|, \quad \forall z \in \mathcal{D}\left(L_{T}^{*}\right) .
$$

A readily accessible step (which may be carried out either at the PDE-level, or at the operator-theoretic level) permits one to recast (0.2) - for each evolution equation of concern, thus for any such $L_{T}$-into an equivalent PDE estimate: there exists a constant $c_{T}>0$ such that

$$
\left\{\begin{aligned}
&\left.c_{T} E_{w}(0) \leq \int_{0}^{T} \int_{\Gamma_{1}} \text { (suitable trace of } w\right)^{2} d \Gamma_{1} d t \\
& w= \text { solution of dual problem defined by } L_{T}^{*}, \text { which is } \\
& \text { homogeneous on the boundary in the same B.C. } \\
& \text { where the boundary control is applied in the original } \\
& \text { problem defining } L_{T} .
\end{aligned}\right.
$$

In (0.3), $E_{w}(t)$ is the 'energy' (norm) associated with the solution $w$ of the dual, boundary homogeneous PDE, whose homogeneous Boundary Condition(s) (B.C.(s)) correspond(s) to the B.C.(s) where the boundary controls are applied in the original problem defining $L_{T}$. Moreover, $\Gamma_{1}$ is the 'observed' portion of the boundary $\Gamma=\partial \Omega=\Gamma_{1} \cup \Gamma_{0}$ of the dual problem $w$. 
This equivalently says that $\Gamma_{1}$ is the 'controlled' portion of the boundary of the original problem; that is, $\Gamma_{1}$ is the part of $\Gamma$ where controls are applied, while on the complementary portion $\Gamma_{0}=\Gamma \backslash \Gamma_{1}$ of the boundary, the boundary conditions are instead homogeneous. Finally, $T$ in $(0.3)$ is a (universal) time for boundary observation of the dual problem $w$, or for boundary controllability of the original problem defining $L_{T}$.

We note at the outset that the vanishing trace (restriction) of the boundary homogeneous solution $w$ is 'complementary' to the trace appearing on the RHS of (0.3). For instance, in the case of the wave equation, if the boundary controls are applied to the Dirichlet [respectively, Neumann] B.C. on $(0, T] \times \Gamma_{1}$, then the dual problem $w$ satisfies homogeneous (zero) Dirichlet B.C. [respectively, Neumann B.C.] on $(0, T] \times \Gamma_{1}$, while the trace of $w$ occurring on the RHS of (0.3) is the Neumann trace [respectively, a Dirichlet trace]. We refer to Part III for specific cases: see (6.5.3) and (6.5.6) below for second-order hyperbolic equations; see (8.14) and (8.17) below for Schrödinger equations, etc.

Inequality (0.3) is traditionally [R.1] called the Continuous Observability Inequality (C.O.I.) of the evolution described by the dual boundary homogeneous $w$-problem (of which $(0.2)$ is the abstract version in terms of $L_{T}$ ).

To recap, we have thus seen that: the exact boundary controllability (surjectivity) property (0.1) is then equivalent to the Continuous Observability Inequality (0.3). [The equivalence between controllability and observability has been noticed and used extensively in the finite-dimensional control theory, since the work of R. Kalman in the early 1960's.]

The crux of the entire problem is, therefore, to establish the validity of the C.O.I. (0.3) [possibly with a "minimal" universal time $T$, with a "minimal" observed/controlled portion of the boundary $\Gamma_{1}$ and, above all] with the optimal relationship between the topology of the trace and the topology of the initial energy. We note that [(0.2) or] $(0.3)$ is an inverse-type inequality: it reconstructs the initial energy of the $w$-evolution equation in terms of information on the boundary (trace, or restriction, of the solution). This establishes a link between this part of control theory and inverse problems. $A$-fortiori, the continuous observability inequality (0.3) implies a global unique continuation result: if the boundary homogeneous $w$-problem has, in addition, equal to zero also the traces on $\Gamma_{1}$ contained on the RHS of (0.3), then $E(0)=0$ and (when the problem is semigroup well-posed forward in time), then $w \equiv 0$ identically. However, the precise nature of the inequality in (0.3) - where the inverse map from the boundary traces (restrictions) over $[0, T] \times \Gamma_{1}$ of the boundary homogeneous problem $w$ to the initial energy is continuous - makes the continuous observability inequality much stronger than the corresponding global unique continuation property of the over-determined problem. The latter is a much studied (and already challenging to study) basic property in pure PDE theory [Lit.2], [Hor.1], [Ta.8], [Tay.1]: however, the C.O.I. (0.3) requires much more! 
Constructive minimal-norm steering control. One issue of potential interest remains. Suppose that one succeeds in showing the C.O.I. (0.3) for the dual problem $w$. Then, equivalently, the original problem is exactly controllable on $[0, T]$, by means of boundary controls applied on $(0, T] \times \Gamma_{1}$. But then: can one construct a boundary control that steers say, the origin [respectively, an original state] at time $t=0$ to a desired target state [respectively, to rest] at $t=T$ ? The answer is in the affirmative: once exact boundary controllability is established (by showing the validity of the dual C.O.I. in (0.3)), one may then set up a minimization problem with constraint, to identify (via a Lagrange multiplier, functional analytic argument) the minimal norm boundary control; this is the control that, among all controls performing the required transfer, has minimal norm. References [Tr.1, Appendix], [L-T.3, Appendix] produce general formulas of the minimal norm, steering controls related to abstract (linear) equations, which encompass the present case of boundary controls for PDEs.

These formulas are as follows. Henceforth, we call $U$ the abstract control space and $e^{\mathcal{A t}}$ the free dynamics s.c. semigroup on the state space. Then, under the property of exact controllability, we have:

(i) The minimal $L_{2}(0, T ; U)$-norm steering control $u^{0}\left(\cdot ; z_{0}\right)$ [respectively, $\left.u_{z_{T}}^{0}(\cdot)\right]$, which steers the initial state $z_{0}$ at $t=0$ [respectively, the origin at $t=0$ ] to the origin at $t=T$ [respectively, to the target state $z_{T}$ at $t=T$ ], along the dynamics described by the operator $L_{T}$, is given by

$$
u^{0}\left(\cdot ; z_{0}\right)=-L_{T}^{*}\left(L_{T} L_{T}^{*}\right)^{-1} e^{\mathcal{A} T} z_{0} \quad\left[\text { resp. } u_{z_{T}}^{0}(\cdot)=L_{T}^{*}\left(L_{T} L_{T}^{*}\right)^{-1} z_{T}\right] ;
$$

(ii) The corresponding minimal control energy is given by

$$
\left\{\int_{0}^{T}\left\|u^{0}\left(t ; z_{0}\right)\right\|^{2} d t\right\}^{\frac{1}{2}}=\left\|\left(L_{T} L_{T}^{*}\right)^{-\frac{1}{2}} e^{\mathcal{A} T} z_{0}\right\|
$$

[respectively,

$$
\left.\left\{\int_{0}^{T}\left\|u_{z_{T}}^{0}(t)\right\|^{2} d t\right\}^{\frac{1}{2}}=\left\|\left(L_{T} L_{T}^{*}\right)^{-\frac{1}{2}} z_{T}\right\| . \quad\right]
$$

Uniform boundary stabilization and equivalent inequality (Linear case). One begins with a conservative (energy preserving) evolution equation, homogeneous on the boundary. Next, one seeks to identify a suitable dissipative (damping) ("feedback") term on (a suitable portion of) the boundary such that: the new corresponding dissipative problem is (i) well-posed (that is, it defines a semigroup $e^{A_{F} t}, F$ stands for feedback) and (ii) its energy ("norm") $E(t)$ decays exponentially to zero: there exist constants $M \geq 1, \delta>0$ such that

$$
E(t) \leq M e^{-\delta t} E(0), \text { equivalently }\left\|e^{A_{F} t}\right\| \leq \bar{M} e^{-\delta t}, t \geq 0 .
$$


A (necessary and) sufficient condition for achieving the exponential energy decay (0.4) is as follows: there exists a time $T>0$ sufficiently large, and a corresponding constant $c_{T}>0$ such that the following Uniform Stabilization Inequality (U.S.I.) holds true:

$$
\left\{\begin{array}{l}
\left.c_{T} E_{y}(T) \leq \int_{0}^{T} \int_{\Gamma_{1}} \text { (suitable boundary dissipation of } y\right)^{2} d \Gamma_{1} d t \\
y=\text { solution of boundary dissipative problem. }
\end{array}\right.
$$

Indeed, (0.5) would then imply (be equivalent to) the property that the resulting feedback semigroup $e^{A_{F} t}$ describing the dissipative system satisfies the condition: $\left\|e^{A_{F} T}\right\|<1$. And this is a well-known characterization for the semigroup $e^{A_{F} t}$ to be exponentially stable (in the appropriate uniform norm).

We have said that, by construction, the $y$-problem is dissipative. This means that it satisfies the following dissipative identity:

(0.6) $E_{y}(T)+\int_{0}^{T} \int_{\Gamma_{1}}(\text { suitable boundary dissipation of } y)^{2} d \Gamma_{1} d t=E_{y}(0)$,

which can readily be proved, by and large, by a routine energy method. Here $\Gamma_{1}$ is the portion of the boundary affected by the dissipation, while homogeneous B.C. are in place on $\Gamma \backslash \Gamma_{1}$. Moreover, the "suitable boundary dissipation of $y$ " appearing in both (0.5) and (0.6) is the same. Thus, in the LHS of the U.S.I. (0.5), we can always replace the energy $E_{y}(T)$ at $t=T$ with the initial energy $E_{y}(0)$, modulo a different constant $c_{T}>0$. Thus, with the U.S.I. $(0.5)$, with either $E_{y}(T)$ or else $E_{y}(0)$ on its LHS, we are back to an inverse-type inequality, this time for the boundary dissipative problem $y$ (not the boundary homogeneous problem $w$, as in (0.3). This is another link between this part of control theory and inverse problems!

Topological level in exact boundary controllability and in uniform boundary stabilization. It is clear that showing the U.S.I. (0.5) is generally more challenging than showing the C.O.I. (0.3). For one, (0.5) refers to a more complicated boundary dissipative problem $y$, while $(0.3)$ refers to a simpler boundary homogeneous problem $w$. In addition, and much more seriously, the energy $E_{y}$ may very well be at a lower Sobolev level than the energy $E_{w}$ in (0.3). This occurs, e.g., in the case of uniform stabilization of either the (conservative) wave equation or the (conservative) Schrödinger equation with dissipative term in the Dirichlet (rather than Neumann) B.C.: for a treatment of wave equations, we refer to the forthcoming Section 6.6). In these cases, the energy level $E_{y}$ of the dissipative $y$-problem is one Sobolev unit below than the energy level $E_{w}$ of the homogeneous case:

(i) $E_{y} \sim L_{2}(\Omega) \times H^{-1}(\Omega)$, see [L-T.1], [L-T.12], [L-T-Y.3]; $E_{w} \sim$ $H^{1}(\Omega) \times L_{2}(\Omega)$ for wave equation; 
(ii) $E_{y} \sim L_{2}(\Omega)$, see [L-T.8]; $E_{w} \sim H^{1}(\Omega)$ for Schrödinger equation.

Showing the inverse type inequality at a lower energy level (below the 'physical' energy) is surely much more demanding. It requires a shift of topology. We refer to Remark 6.6.2 for more technical and historical insight.

Relationship between exact boundary controllability and uniform boundary stabilization. There is a two-way relationship between uniform boundary stabilization and exact boundary controllability of an evolution equation of the type under consideration.

In one direction, for an originally, say conservative problem, a simple, smart idea - due to D. Russell [R.1] — shows that uniform stabilization (for positive times) of the new dissipative problem implies exact controllability of the original conservative evolution equation, on the same function space, constructively, with a steering control which may be constructed by means of two stabilizing feedback controllers. As a result of this argument, the transfer from the initial state to the target state of the original conservative system takes place continuously in time with values in the state space: a bonus or desirable property this, that cannot follow from the C.O.I. (e.g., exact controllability in $H^{1}(\Omega) \times L_{2}(\Omega)$ of the wave equation with $L_{2}\left(0, T ; L_{2}(\Gamma)\right)$-Neumann controls), since the latter is only concerned with what happens at $t=T$ and not with the evolution over $[0, T]$, which is a regularity question. The additional price paid in this approach - where one begins by proving the more demanding U.S.I. (0.5) rather than the less demanding C.O.I. (0.3) is twofold: (i) the original system has to be conservative (a small generalization is possible); (ii) the (universal) time for exact controllability is generally much larger than necessary.

In the other direction, consider a general evolution equation which is exactly controllable on the space of optimal regularity: this means that both the C.O.I. (0.3) (possibly for $T$ large) as well as its reverse inequality (for any $T>0$ ) hold true (in the same topologies for the initial energy and boundary traces). Then the theory of optimal control with a quadratic cost functional to minimize provides a different feedback stabilizer, not expressed as a velocity feedback as discussed heretofore, but expressed by means of a Riccati operator: but this is an altogether different topic, for which we refer to [L-T.11], [L-T.24]. A recent related paper on these questions is [L-T.30].

Thus, this part of control theory for PDEs concerned with exact controllability and uniform stabilization seeks to establish the validity of the equivalent dual inequalities: the C.O.I. (0.3) and/or the U.S.I. (0.5). [There are some exceptions which follow a direct approach to exact controllability (not via the dual problem): (i) the use of harmonic or non-harmonic analysis, moment problems, Riesz basis, almost exclusively in one-dimensional problems (or problems reducible to one dimension by spatial symmetry) and thus not in the mainstream interest of the present article; (ii) the approach of W. Littman [Lit.1], [Lit-Ta.1] inspired by the Huyghens' prin- 
ciple. Though these concepts of exact controllability/uniform stabilization are very 'old,' it was only in the early mid-1980s that inequalities such as the U.S.I. (0.5) (or equivalent versions) and the C.O.I. (0.3) were established, at first for the pure Euclidean wave equation: with the Euclidean Laplacian and no energy level terms $\left(H^{1}(\Omega) \times L_{2}(\Omega)\right.$, that is, gradients in space and time). Soon thereafter, the successes achieved with the pure Euclidean wave equation, were transferred to establish the corresponding inequalities - C.O.I. (0.3) and U.S.I. (0.5) - also for the pure Euclidean Schrödinger equation, plate equations (of both Euler-Bernoulli type or of Kirchhoff type), etc., with energy method techniques inspired by the pure Euclidean wave equation case.

Inadequacy of, and difficulties with, "classical" energy methods ("multipliers") of the ' $80 \mathrm{~s}$ in the case of variable coefficients and energy level terms. By the end of the 80 's, one may say that the energy methods used (see the many works cited in the References) (special multipliers), however, worked well only for such canonical wave/Schrödinger/plate models (constant coefficients and no energy level terms), but were inadequate to treat more general models with variable coefficients in the principal part and/or in the energy level terms. This brings us to the main motivation of the present article: How to establish the C.O.I. (0.3) and the U.S.I. (0.5) for single PDEs, or coupled systems of PDEs, with variable coefficients in both the principal part and in energy level terms and/or defined on curved surfaces? Whatever method is used, this will have to represent a seriously more technical and sophisticated approach over the "classical" one of the ' 80 's, which was successful only for canonical models. One general line of investigation proposed relies on Riemannian geometry: its energy method approach - to be expounded in Section 3 and Part III - may be viewed as a far-reaching generalization of the energy methods of the ' 80 's. This will be made more clear in Remark 6.4.1 below for second-order hyperbolic equations; in Remark 8.1 for Schrödinger equations; and in Remark 10.1 for plate-like equations.

Other approaches to the control of variable coefficient (linear) PDEs include: (i) the geometric optics line of investigation [Lit.1], [B-L-R.1] for second-order hyperbolic equations, with $C^{\infty}$-domains and with $C^{\infty}$ coefficients (later reduced to $C^{3}$-domains and $C^{2}$-coefficients in [Bu.1] via the use of $H$-measures, but only for the variable coefficient pure wave equation with no energy level terms and Dirichlet control). It is likely that recent improvements in the field of propagation of singularities [Tay.2] will allow to reduce the degree of smoothness required by [Lit.1], [B-L-R.1]; (ii) the pseudo-differential method in [Ta.1-3] for general evolution equations. The Ph.D. thesis [Ta.1] (for general evolution equations) and [K-K.1] (second-order hyperbolic equations with constant principal part and traditional B.C.) were, apparently, the first works to introduce in the control literature Carleman estimates involving boundary traces, the object of key interest in control-theoretic inequalities. Prior literature on the vast topic 
of Carleman estimates (initiated by Carleman around 1937 and much expanded and perfected by Hörmander [Hor.1-2], Taylor [Tay.1]) referred to solutions of PDEs with compact support for purposes of unique continuation, and hence contained no boundary traces. There appears to be a close link [Hor.1], [Ta.8], in both scope and formulation of hypotheses, between the pseudo-differential energy method approach of [Ta.1-3] and the Riemannian energy method of Part III (Sections 6, 8, 10), though the mathematical machineries and technicalities involved are vastly different. The infusion of Riemannian geometric ideas does appear to help, at any rate, to clarify and verify the notions of pseudo-convexity in the pseudo-differential approach. See further Remark 3.2.1 below, and Sections 4 and 5 .

1. Introduction: Aim and scope of the present paper. Qualitative statement of results. Two approaches.

1.1. Linear single PDEs, or coupled systems of PDEs, with variable coefficients and/or defined on curved surfaces. Very recently, differential (Riemannian) geometric methods have been introduced in the control of certain broad classes of Partial Differential Equations (PDEs). As explained in the preceding historical summary, the original motivation may be said to have arisen from the need to cope with the following two situations:

(i) The case where the coefficients of the PDE are variable in space in its principal part and, possibly, variable in both space and time in its 'energy level' terms; ifolds).

(ii) the case where the PDE itself is defined on curved surfaces (man-

Regarding the first need (i), we shall present two approaches, see Section 1.2: one applicable to various single PDEs, be they hyperbolic or not, as well as to coupled systems of various PDEs; and one specifically tuned to hyperbolic problems. As to the second need (ii), it would seem selfexplanatory that if an equation is defined on a manifold (as in the case of shells), the natural setting which is called for its analysis should be that of differential geometry. However, this was not the case in the large literature in shell theory.

While a mutually profitable link had been long established, for at least 30 years, between differential geometry and control of nonlinear ordinary differential equations, a comparable relationship between differential geometry and control of PDEs is a new topic, which has already confirmed, in just a few years, several of its original promises. Similarly, a useful interplay between differential geometry and the general theory of PDEs has long been in place and documented [Taylor, Hörmander, Treves]. Yet, the distinguished use of differential geometric methods in the setting which arises in boundary control theory of PDE is novel. Moreover, such an approach is also far from having been exhaustively explored. Nevertheless, to date, a relatively large and useful body of knowledge has emerged over the past 
few years in the area of control of PDEs, which is based on differential geometric methods. It was originated in [Y.1] for the purely wave equation followed by [L-T-Y.1-2] for the full second-order hyperbolic equation case.

Main aim. Thus, the main aim of the present paper is to give an updated and motivated account of this direction of research (within space limits). In the process, we intend to make a strong case illustrating the positive role that differential geometric methods may play in the modern study of control of PDEs. In substantiating their usefulness, we shall see that their impact has been, so far, three-fold:

(1) in facilitating, indeed in contributing to, the very mathematical formulation of PDE models defined on curved surfaces (manifolds) such as shells, see Part IV;

(2) in obtaining certain a-priori inverse-type inequalities, such as they are needed in control theory of PDEs, be they hyperbolic or Petrowski-type, with emphasis on variable coefficients and/or on equations defined on manifolds, see Part III.

(3) in the case of second-order hyperbolic equations, in obtaining geometric conditions sufficient to verify the geometric optics condition. See Section 5.

Inverse-type inequalities in control theory: The C.O.I. (0.3) and the U.S.I. (0.5). We shall have to say more below on both points (1), (3). For now, we note that the a-priori inequalities mentioned in (2) are those traditionally referred to as "continuous observability inequalities" (equivalent to corresponding "exact controllability" properties) and "uniform stabilization" inequalities, which were noted in (0.3) and (0.5) of Section 0 . These will be the object of Part III below for different classes of evolution equations.

A preliminary step: Carleman-type inequalities. The inversetype C.O.I. and U.S.I. of interest in control theory will be obtained as corollaries of Carleman-type inequalities (one-parameter family of inequalities with exponential weight) of interest in their own sake. For instance, in Sections 7 and 9, Carleman-type inequalities will, a-fortiori, imply global uniqueness of over-determined PDE-problems. The key Carleman inequalities are given in Theorem 6.4.2 (with lower-order terms) and in Theorem 7.2 (without lower-order terms) for second-order hyperbolic equations; in Theorem 8.2 (with lower-order terms) and in Theorem 9.1 plus [L-T-Z.2] (without lower-order terms) for Schrödinger equations; and in Theorem 10.2 (with lower-order term) for plate-like equations.

1.2. Two geometric approaches. In this paper we shall review recent Riemannian geometric lines of research for PDEs with variable coefficients as above, or else on manifolds. Two quite different approaches will be presented.

Approach 1. One line of research, which wholly pervades the present article, is 'computational' in flavor. It is entirely self-contained. More- 
over, it is broad in scope, as it applies to both hyperbolic as well as nonhyperbolic, Petrowski-type classes of PDEs, as well as to coupled systems of such PDEs (e.g., structural acoustic chambers as in Section 2; shells as in Section 11). Its virtue is that, as noted above, it yields general Carlemantype estimates from which observability/stabilization estimates $(0.3)$ or (0.5) may be derived. They are obtained by using energy methods (multipliers) in a corresponding natural Riemannian metric. These multipliers may be viewed as far-reaching extensions of the 'classical multipliers' of the early 80's used in the Euclidean setting for 'canonical' PDEs, with constant principal part and no energy level terms in the equation, as noted in Section 1. See Remark 6.4.1 for second-order hyperbolic equations; Remark 8.1 for Schrödinger equations, and Remark 10.1 for plate-like equations. In addition, the combination of these Riemannian methods with microlocal sharp trace estimates will be given in Part IV in solving the stabilization problem of a shallow shell with non-linear, dissipative terms in the physically important free B.C.

The Riemannian geometric multipliers to be exhibited in Part III for different classes of PDEs have several advantages: they allow for a readily accessible geometric interpretation of the key assumption (H.1) $=(3.1 .1)$ behind this method; they open up the vast reserves of differential geometry for the verification of this assumption (H.1) = (3.1.1) and for constructing classes of nontrivial examples (see Section 4); they reveal themselves as being far-reaching generalizations of the 'classical' multipliers of the '80s, to which they reduce in the case of canonical PDEs with constant coefficient principal part and no energy level terms. See Remark 6.4.1, 8.1, and 10.1 for waves, Schrödinger, and plate equations.

Approach 2. Another line of research is more 'geometric' in flavor. It will be presented in Section 5. It offers a 'view-from-the-boundary approach,' which focuses on geodesics. As such, it is confined to hyperbolic problems, where geodesics are (graph of) the bi-characteristics (carriers of energy). This way, this Riemannian approach merges with the established geometric optics approach for hyperbolic problems [Lit.1], [B-L-R.1]. Indeed, this approach produces specific sufficient conditions, which eventually permit the verification of the geometric optic assumptions and hence the application of the geometric optics results.

A comparison between the two methods on the common ground of applicability - hyperbolic equations - is also given in the examples of Section 5.4 .

1.3. Summary of main features of differential geometric methods in the control of PDEs. Over the past $4-5$ years, differential (Riemannian) geometric methods have emerged as a powerful new line of research to obtain general inverse-type, a-priori inequalities of interest in boundary control theory (continuous observability/stabilization inequalities (0.3), (0.5)) for various classes of PDEs. Their range of applicability 
now includes: second-order hyperbolic equations; Schrödinger-type equations; various plate-like equations; systems of elasticity; very complicated shell models described more below in Part IV, etc., all with variable coefficients, where the 'classical' energy methods of the early/mid-80's proved inadequate, as pointed out repeatedly in Section 0. We refer once more to Remark 6.4.1 for second-order hyperbolic equations; Remark 8.1 for Schrödinger equations; Remark 10.1 for plate-like equations.

In all of these PDEs classes, main features of these differential geometric methods are: (1) they apply to operators with principal part which is allowed to have variable coefficients (in space) with low regularity, $C^{1}$; (2) they tolerate energy level terms which are both space- and time-dependent, and only in $L_{\infty}$ in time and space; (3) they yield rather general and verifiable sufficient conditions, which may serve for the construction of many complicated, variable coefficient examples, as well as for counterexamples (say, in the hyperbolic case, in dimension greater than 2), even when the control acts on the whole boundary; see Part II, Section 5; (4) they provide a good estimate (for some classes, optimal estimate) of the minimal time for observability/exact controllability in the hyperbolic case, and arbitrary short time when there is no finite speed of propagation; (5) they combine well with microlocal analysis methods needed for sharp trace estimates and for shifting topologies, thus producing at the end very general observability/stabilization results, with variable coefficients and with no geometric conditions on the observed (controlled) portion of the boundary; see Section 11, Part IV, on shells; (6) they merge well with techniques for reducing the number of traces in plate-like problems [L-T.14], [L-T-Z.2]; (7) ultimately, and with the same effort, they apply to these classes of PDEs defined on Riemannian manifolds, as is often the case in mathematical physics.

In addition, differential geometric methods have recently provided the intrinsic language for: (i) modeling the motion of dynamic shells far beyond the classical approach (rooted in classical geometry), and (ii) performing observability/stabilization energy methods on their very complicated equations, for which the classical setting based on Christoffel symbols appears to be too complicated or unfeasible. As we shall see in Part IV, a shell is a curved geometric object which can be modeled as a system of two PDEs both of hyperbolic type with strong coupling depending on the curvature: an 'elastic wave-type' equation ('curved system of elasticity') in the in-plane displacement; and a 'curved Kirchhoff plate-like equation' for the vertical displacement.

1.4. Geometric methods and control of PDEs: A two-way interaction. Although the subject of boundary control of PDEs is about a quarter of a century old, and that of Riemannian geometry is much older still, there has been relatively little interaction between the two. It was just over 10 years ago that the role that bi-characteristics play in 
boundary control of hyperbolic PDEs was brought to the forefront [Lit.1], [B-L-R.1]. This then naturally leads one to think about their geometric equivalent (Duff [Du.1, p. 209], Courant-Hilbert [C-H.1]), at least for timeinvariant, second-order hyperbolic equations - geodesics, a basic concept in Riemannian geometry. On the other hand, it will be documented below that differential geometric methods play an equally positive role for nonhyperbolic, Petrowski-type PDEs, such as Schrödinger equations, plate-like equations of Euler-Bernoulli type, which have no finite speed of propagation, and where the above critical identification between bi-characteristics and geodesics is no longer available. This aspect is critical when dealing with coupled systems of PDEs of various type, be they hyperbolic or notmore on this later. Each of the two disciplines - control theory of PDEs, and Riemannian geometry - has been pursued in virtually complete independence, or occasionally even ignorance, of the other. It is the belief by the authors that both subjects have much to gain by closer interaction with one another. In this spirit, the authors are pleased to contribute the present paper, in the hope that it will further stimulate a two-way interaction between control of PDEs and differential geometry. On the one hand, we anticipate that the reservoir of as yet untapped Riemannian geometric methods and concepts could be applied productively in boundary control of PDEs. On the other hand, we expect that certain problems which have arisen recently in the boundary control of PDEs will stimulate the disciplines of Riemannian and Lorentzian geometry to undertake new areas of research. One example is the discovery of additional sufficient conditions (over available literature) - to be collected in Section 4 below-which guarantee the existence of a $C^{2}$-strictly convex function on a bounded domain $\Omega$ of a finite-dimensional Riemannian manifold $(M, g)$, where strict convexity is intended with respect to the Riemannian metric $g$. Such strictly convex function plays a critical role in the results presented below in Part III for both hyperbolic as well as non-hyperbolic, Petrowski-type PDEs. A new result of this type, stimulated precisely by the interaction with boundary control theory is given in Theorem 4.6.1 of Section 4.6.

1.5. Systems of coupled PDEs. Nonlinear problems. The advantage of both obtaining explicit estimates, such as the C.O.I. (0.3) or the U.S.I. (0.5), as well as devising the techniques leading to these, is accrued also in two important cases, beyond the original linear single PDE setting: (i) the case of systems of coupled PDEs possibly of different type, to be illustrated in Section 2 below; and (ii) the case of nonlinear (semilinear) versions of the single PDE equations (which we do not treat here for lack of space). To elaborate further, more specifically, global exact controllability results of nonlinear (semilinear) wave and plate problems are obtained with the help of explicit, accurate continuous observability estimates for the corresponding linear problems [L-T.7]. In this work, global exact boundary controllability results are established for wave equations across all state 
spaces $H^{\gamma}(\Omega) \times H^{\gamma-1}(\Omega), 0 \leq \gamma \leq 1, \gamma \neq \frac{1}{2}$, including the most relevant cases at the end points $H^{1}(\Omega) \times L_{2}(\Omega)(\gamma=1)$ and $L_{2}(\Omega) \times H^{-1}(\Omega)$ $(\gamma=0)$. Inclusion of the two cases of greatest interest motivated [L-T.7], as previous work [Z.1] had to exclude precisely the two endpoint cases.

Additional instances where explicit continuous observability estimates have a beneficial impact on nonlinear problems may be given. First, in [LO.1] the proof of global existence of solutions to quasi-linear wave equations depends critically on the continuous observability inequality of the linear part. Second, in [Las.5], [Las.6], continuous observability estimates for the linear components are critically used to prove uniform stabilization of the (nonlinear) von Karman equation. Third, Part IV will give a version of this same strategy in the case of a shell: the continuous observability inequality of its linear part [Y.4] will be needed to prove-in the style of [Las.5], [Las.6] - uniform stabilization of a shallow shell [L-T.29] by virtue of nonlinear dissipative terms in the physically relevant and mathematically challenging Free Boundary Conditions. We finally refer to the books of Lagnese [Lag.3] and Komornik [K.1] for further instances on the use of C.O.I. for the stabilization of semi-linear problems.

2. A benchmark PDE-control problem advocating a broad use of differential geometry: The structural acoustic problem.

General considerations. The structural acoustic problem-to be described below in its various forms - provides an excellent carrier to motivate, introduce, justify and advocate the use of differential geometry in boundary control theory for PDEs. Indeed, realistic mathematical models of structural acoustic problems consist of a second-order hyperbolic equation (modeling the acoustic pressure) defined on a bounded domain $\Omega$ of $\mathbb{R}^{3}$, where the restriction of its solution to its elastic wall $\Gamma_{0}$ couples with an elastic equation (modeling its deflection) defined on $\Gamma_{0}$. In turn, the elastic solution couples with the boundary conditions of the second-order hyperbolic equation as expressed on $\Gamma_{0}$. The complementary part $\Gamma_{1}$ of the boundary of $\Omega$ is, instead, rigid. The elastic equation defined on $\Gamma_{0}$ may be of various types: (i) a hyperbolic elastic PDE of Kirchhoff type with, or without, strong damping; (ii) a non-hyperbolic elastic PDE of EulerBernoulli type with, or without, strong damping, (iii) an elastic PDE of either Kirchhoff type or Euler-Bernoulli type which, in addition, accounts for thermal effects, thus becoming a thermoelastic system on $\Gamma_{0}$, and thus coupling, in turn, an elastic and a heat equation on $\Gamma_{0}$; (iv) a composite (sandwich) plate. Finally, the elastic wall may either be flat, in which case the aforementioned equations in (i) to (iv) are elastic or thermoelastic or sandwich plates; or else may be curved (a manifold), in which case the aforementioned equations in (i) to (iv) are elastic (dynamic) or thermoelastic or composite shells. In realistic environments, where properties of the medium vary from point to point, the corresponding PDEs have space variable coefficients, even in the flat case. The above qualitative description, therefore, 

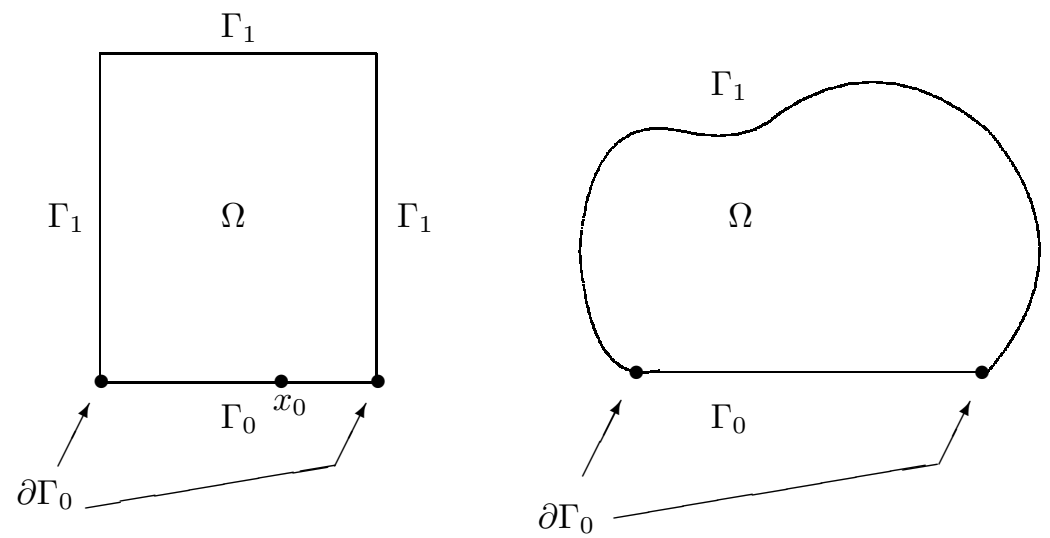

FIG. 1.

catapults up front some of the key features that the present paper intends to address and emphasize. They are: (i) second-order hyperbolic equations with variable coefficients described on a bounded Euclidean domain;(ii) elastic plate-like equations, both hyperbolic-like (Kirchhoff type) and not (Euler-Bernoulli type), with variable coefficients, defined on a Euclidean domain (such as the flat wall $\Gamma_{0}$ ); (iii) thermoelastic plate-like equations, both with hyperbolic or non-hyperbolic elastic component, and with variable coefficients, defined on a Euclidean domain (such as the flat wall $\Gamma_{0}$ ); (iv) (dynamic) elastic and thermoelastic shell equations defined on a curved surface (manifold), such as the curved wall $\Gamma_{0}$.

As we shall see in Part IV, an elastic shell equation is a system of two coupled hyperbolic-like PDEs, defined on a curved surface, which comprises: (i) the system of elasticity in the in-plane 2-dimensional displacement of the shell; and (ii) a scalar Kirchhoff type plate-like equation in the normal displacement. In the present paper, to the above list, we shall add one more dynamics: (v) Schrödinger equations with variable coefficients defined on a Euclidean domain (the iteration of two Schrödinger equations yields a plate-like equation).

2.1. Various structural acoustics models with constant coefficients and with flat flexible wall.

The acoustic chamber. Let $\Omega \subset \mathbb{R}^{3}$ be an open bounded domain ("the acoustic chamber") with boundary $\Gamma=\overline{\Gamma_{0} \cup \Gamma_{1}}$, where $\Gamma_{0}$ and $\Gamma_{1}$ are open, connected, disjoint parts, $\Gamma_{0} \cap \Gamma_{1}=\emptyset$ in $\mathbb{R}^{2}$, of positive measure. Throughout this subsection, the sub-boundary $\Gamma_{0}$ is flat and is referred to as the elastic or flexible wall. Instead, $\Gamma_{1}$ is referred to as the rigid or hard wall. The interaction between wave and plate in the models below takes place on $\Gamma_{0}$. We also assume throughout that either $\Omega$ is sufficiently smooth 
(say, $\Gamma$ is of class $C^{2}$ ), or else $\Omega$ is convex. This assumption guarantees that solutions to classical elliptic equations with $L_{2}(\Omega)$-forcing terms are in $H^{2}(\Omega)$ [Gri.1], or that the domain of the Laplacian in $\Omega$, with (either Dirichlet or) Neumann B.C., is contained in $H^{2}(\Omega)$. The acoustic medium in the chamber is described by the wave equation in the variable $z$ with acoustic pressure $\rho_{1} z_{t}$, where $\rho_{1}$ is the density of the fluid. Moreover, we let $c^{2}$ be the speed of sound. Finally, $v$ denotes the deflection of the plate equation on $\Gamma_{0}$.

Structural acoustic model with elastic flat wall $\Gamma_{0}$ : Hyperbolic/hyperbolic interaction. Here the mathematical model is given by the following coupled PDE system:

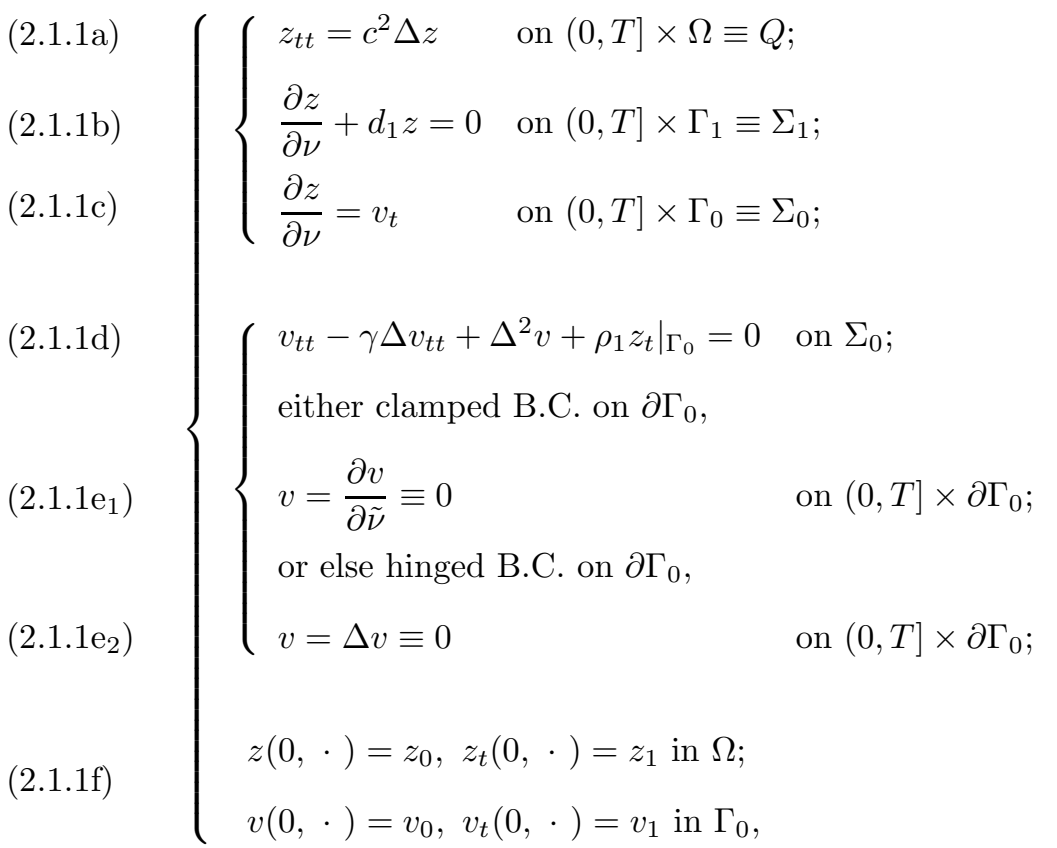

where $\nu(x)=$ unit outward normal vector at $x \in \Gamma$ in $(2.1 .1 \mathrm{~b}-\mathrm{c})$ and similarly for $\tilde{\nu}(x)$ at $x \in \partial \Gamma_{0}$ in $\left(2.2 .1 \mathrm{e}_{1}\right)$ and $d_{1}>0$ (constant). Moreover, in the present model $\gamma>0$ (constant), so that the uncoupled $v$-equation in $(2.1 .1 \mathrm{~d})$, with $\rho_{1}=0$, is the hyperbolic Kirchhoff equation. Naturally, one may supplement the $v$-plate equation (2.1.1d) with other B.C.'s, such as the complicated Free B.C. [Lag.1-3]. [L-T.24, Chapter 3].

Structural acoustic model with thermoelastic flat wall $\Gamma_{0}$ : Hyperbolic/parabolic coupling $(\gamma=0)$ or hyperbolic/hyperbolictype coupling $(\gamma>0)$. Here, the mathematical model is given by the following doubly coupled PDE system: 
$(2.1 .2 \mathrm{a})$

$$
\begin{cases} \begin{cases}z_{t t}=c^{2} \Delta z & \text { on }(0, T] \times \Omega \equiv Q ; \\ \frac{\partial z}{\partial \nu}+d_{1} z=0 & \text { on }(0, T] \times \Gamma_{1} \equiv \Sigma_{1} ; \\ \frac{\partial z}{\partial \nu}=v_{t} & \text { on }(0, T] \times \Gamma_{0} \equiv \Sigma_{0} ;\end{cases} \\ \begin{cases}v_{t t}-\gamma \Delta v_{t t}+\Delta^{2} v+\Delta \theta+\left.\rho_{1} z_{t}\right|_{\Gamma_{0}}=0 & \text { on } \Sigma_{0} \\ \theta_{t}-\Delta \theta-\Delta v_{t}=0 & \text { on } \Sigma_{0}\end{cases} \end{cases}
$$

Boundary Conditions, such as the clamped or hinged B.C. (2.1.1e) for $v$ and, say, the thermal Dirichlet condition $\theta \equiv 0$ in $(0, T] \times \partial \Gamma_{0}$ need to be added, along with initial conditions $\left\{z_{0}, z_{1}, v_{0}, v_{1}, \theta_{0}\right\}$.

For $\gamma=0$, the $\{v, \theta\}$-dynamics (2.1.2d-e) with $\rho_{1}=0$ generates a s.c. analytic semigroup on a natural (energy) state space based on the flat wall $\Gamma_{0}$, under all canonical B.C., including the complicated coupled free B.C. for $\{v, \theta\}$ [L-T.18-20], [L-T.24, Appendices to Chapter 3]. Instead, for $\gamma>0$, the $\{v, \theta\}$-dynamics $\left(2.1 .2 \mathrm{~d}\right.$-e) with $\rho_{1}=0$ is hyperbolic-dominated, in the technical sense of [L-T.27].

2.2. More realistic models: Variable coefficients and curved walls. More realistic models demand the need of further refining the canonical models of Section 2.1, by introducing additional non-trivial complications. In order of progressively increasing difficulties, they are:

(i) still in the case of flat wall $\Gamma_{0}$, the differential operators, in particular the Laplacian $\Delta$ (both on $\Omega$ as well as on $\Gamma_{0}$ ) should be replaced by variable coefficients elliptic operators, to reflect the fact that properties of the medium may vary from point to point. In particular,

$\left(i_{1}\right)$ the wave equation (2.1.1a) should be replaced by its variable coefficient counterpart

$$
z_{t t}+\mathcal{A} z=F(z) \text { in }(0, T] \times \Omega \equiv Q,
$$

where for some positive $c>0$ and $a_{i j}(x)=a_{j i}(x) \in C^{1}(\Omega)$,

$$
\begin{gathered}
\mathcal{A} z \quad \equiv-\sum_{i, j=1}^{3} \frac{\partial}{\partial x_{i}}\left(a_{i j}(x) \frac{\partial z}{\partial x_{j}}\right) ; \\
\sum_{i, j=1}^{3} a_{i j}(x) \xi_{i}, \xi_{j} \geq c \sum^{2} \xi_{i}^{2}, x \in \bar{\Omega} \\
F(z)=q_{1}(t, x) \cdot \nabla z+q_{2}(t, x) z_{t}+q_{0}(t, x) z ; \\
\left|q_{1}\right| \in L_{\infty}(Q) ; q_{2} \in L_{\infty}(Q), q_{0} \in L_{p}(Q), p=n+1=4 .
\end{gathered}
$$


$\left(\mathrm{i}_{2}\right)$ The thermo-plate-equation $(2.1 .2 \mathrm{~d})$ should be replaced by its variable coefficient counterpart [E-L-T.1-4]:

$$
\left\{\begin{aligned}
v_{t t}-\gamma \mathcal{A} v_{t t}+\mathcal{A}^{2} v+\operatorname{div}(\alpha(x) \nabla \theta)+\left.\rho_{1} z_{t}\right|_{\Gamma_{0}} & =0 \\
\theta_{t}-\mathcal{A} \theta-\operatorname{div}\left(\alpha(x) \nabla v_{t}\right) & =0
\end{aligned}\right.
$$

(ii) The flat wall $\Gamma_{0}$ should give rise to a curved wall $\Gamma_{0}$, so that the elastic or thermoelastic equations in (2.1.1d) and (2.1.2d) respectively, should be replaced by (dynamic) elastic shell and thermoelastic shell systems. A mathematical model of an elastic shell system is given in Section 11 of Part IV below.

2.3. Conclusion. We have seen that a realistic model of a structural acoustic chamber consists of a system of two or more coupled PDEs, all with variable coefficients, defined on different domains, one of which is a curved wall. Accordingly, this model offers a persuasive case of the need of employing a unifying mathematical machinery, equally capable of treating single PDEs of various type, hyperbolic or not, and equally at ease on either a Euclidean domain or a curved surface. A main point of our present paper is that the Riemannian geometric methods presented here represent an ideal mathematical tool for this purpose. This is documented in the survey of single PDEs in Part III as well as in the treatment of a dynamic shell in Part IV. It will take us two far afield, however, to review the mathematical literature of a structural acoustic chamber [Las.1,7], [L-T.25], which is presently mostly restricted to constant coefficient models with flat flexible walls as in Section 2.1.

The literature is extensive. It includes many works by the second and fourth author of the present paper, and their coworkers (G. Avalos, C. Lebiedzik, etc.)

Part II: Riemannian geometric assumptions. Overview of results: Carleman estimates $\Rightarrow$ C.O.I. and U.S.I.

Orientation. Two geometric approaches: (1) general energy methods in a Riemannian metric for hyperbolic or Petrowski type PDEs, and (2) a view from the boundary for second-order hyperbolic equations. (1) A main geometric approach which has been devised in recent years to obtain the sought-after inverse-type inequalities (0.3) and (0.5) of interest in control theory of PDEs is an 'energy method.' As such, it is a computational method, which is carried out in a suitable Riemannian metric. Indeed, we shall present two versions of it, which are progressively more flexible and hence lead to progressively more desirable results. They are also progressively more complicated. They have been so far successfully tested in several of the desirable PDE evolution equations, whether hyperbolic or not. At any rate, in both versions, the 'energy method' relies, principally, on just one main geometric, fairly general assumption, which 
is in common to, and shared by, all specific classes of evolution equations here considered: second-order hyperbolic equations; Schrödinger equations; plate-like equations of either Kirchhoff type (hyperbolic), or else of EulerBernoulli type (non-hyperbolic). This assumption postulates the existence of a strictly convex function on the open bounded set $\Omega$ of interest of the finite-dimensional Riemannian manifold $(M, g)$ - hence roughly an upper or a lower bound on the sectional curvature. More precisely, we can construct a convex function assuming (i) upper bounds on the sectional curvature, starting out from a point, or a concave piece of the boundary; or (ii) lower bounds on the sectional curvature, starting out from a convex part of the boundary, or from infinity. Once this assumption is satisfied on $\Omega$, then the corresponding control problem of exact controllability and uniform stabilization are generally always solvable on $\Omega$, by using control action on a sufficiently large portion of the boundary, regardless of the specific class of evolution equation which is defined on $\Omega$, hyperbolic or not. There are additional key features in this energy method: (i) the topology of the obtained estimates is optimal, a critical property, for these results to be useful in optimal control theory [L-T.11], [L-T.24]; (ii) the (universal) time in the estimates is often optimal (but not always, e.g., in some classes of hyperbolic problems, where, at any rate, is explicitly estimated, see (3.2.2) below); (iii) the portion of the boundary required (though not necessarily minimal) is, however, explicitly determined by the assumed convex function $v$ as that part of $\Gamma$ where the explicit vector field $\nabla_{g} v$ is entering; (iv) the regularity of the coefficients required is essentially optimal, etc. Last but not least, this geometric assumption permits one to construct many non-trivial PDE examples with variable coefficients, and is, in many cases, checkable. See Section 4. These characteristics may compensate for the fact that it may not be the 'most general.' It appears to be fairly general, however. An exception where a further geometrical restriction may be required is the 'purely Neumann problem' for second-order hyperbolic equations, of which we will have to say more in Section 7.

(2) For the class of second-order hyperbolic equations with Dirichlet (or Neumann) boundary control, we shall present an alternative approach, which we label "a view from the boundary." In it, no direct hypothesis on the Riemannian metric in the interior of $\Omega$ is required, unlike the previously discussed energy method approach. In the "view-from-the-boundary" approach, key emphasis is focused on the geodesics of $(M, g)$, which are the geometric version of the geometric optic notion of bi-characteristics (Duff [Du.1, p. 209], Courant-Hilbert [C-H.1]). For second-order hyperbolic equations, the bi-characteristics are the true carriers of energy of the solutions. The assumptions required by the "view-from-the-boundary" approach will be introduced and discussed in Section 5, along with its main features. Ultimately, they are shown to be sufficient to guarantee the geometric optics condition [B-L-R.1], [Lit.1]. 
3. Basic geometric assumption for the energy method approach in the Riemannian metric.

3.1. Strictly convex functions and coercive fields in the Riemannian metric for hyperbolic and non-hyperbolic (Petrowski)problems. Regardless of the specific class of evolution equations here considered-second-order hyperbolic equations; Schrödinger equations; plate-like equations of either Kirchhoff type (hyperbolic), or else EulerBernoulli type (non-hyperbolic) - the basic assumption that is common to all these different classes in the energy method approach (to be described below) is the following.

Main assumption (H.1). Let $(M, g)$ be a finite-dimensional Riemannian manifold. Let $\Omega$ be an open bounded connected subset of $M$.

We assume that there exists a function $v_{0}: \bar{\Omega} \rightarrow \mathbb{R}$ of class $C^{2}$ which is strictly convex on $\bar{\Omega}$, with respect to the Riemannian metric $g$. We then set, henceforth, $v(x) \equiv v_{0}(x)-\min _{x \in \bar{\Omega}} v_{0}(x) \geq 0$.

More specifically, this means the following. Denote by $D$ the LeviCivita connection in the Riemannian metric $g$. Then, a quantitative version of this assumption is that there exists a positive constant $\rho>0$, such that

$$
D^{2} v(X, X) \equiv\left\langle D_{X}(D v), X\right\rangle_{g} \geq 2 \rho|X|_{g}^{2}, \quad \forall x \in \Omega, \quad \forall X \in M_{x}
$$

In words: for each point $x \in \Omega \subset M$ and each vector field $X$ in the tangent space $M_{x}$ at $x$, the Hessian $D^{2} v$ (a 2-covariant tensor) is coercive, uniformly in $x \in \Omega$. Since $\bar{\Omega}$ is compact in $M$, this assumption means that the Hessian $D^{2} v$ of $v$ in the Riemannian metric $g$ is positive on $\bar{\Omega}$ : $D^{2} v(X, X)(x)>0, \quad \forall x \in \bar{\Omega}, \quad \forall X \in M_{x}$.

We finally note that for a scalar function $v$, we have $D v=\nabla_{g} v$, where $\nabla_{g}$ is the gradient in the Riemannian metric $g$. Assumption (3.1.1) was introduced in [L-T-Y.1-2].

Condition (3.1.1) on the Hessian tensor of $v$ has the following geometric interpretation: for any geodesics $\gamma(s)$ parametrized by arc lengths, then the second derivative in $s$ of $v(\gamma(s))$ satisfies: $v(\gamma(s))^{\prime \prime} \geq 2 \rho$.

The above geometric interpretation of (3.1.1) appears to be related to the notion of pseudo-convexity arising in the framework of pseudodifferential analysis [Hor.1-2]. For instance, for a real non-elliptic symbol $p$ of a partial differential operator $P$, the notion that " $v$ is strictly convex with respect to the null bicharacteristic flow of $P$ near its critical points," is expressed by Poisson bracket conditions, e.g., [Ta.8, Section 2].

We shall devote all of Section 4 to the issue of verifying assumption $($ H.1 $)=(3.1 .1)$.

A first candidate for a suitable $v$ verifying (3.1.1) is $v(s)=d_{g}^{2}\left(x, x_{0}\right), d_{g}$ distance function in the $g$-metric: this, however, need not be strictly convex on some $\Omega$ in general. It becomes strictly convex under some additional properties of the sectional curvature, see Section 4. 
Remark 3.1.1. (1) Assumption (H.1) = (3.1.1) will be our sole assumption to obtain what we call "Carleman estimates, first version," for any of the classes of PDEs in full generality mentioned above, without any boundary conditions (B.C.) being imposed; see Theorem 3.2.1 below as well as Part III. Such "first version of Carleman estimates" is, in each case, the preponderant part of the proof to obtain the sought-after inverse-type inequalities (0.3), (0.5) (continuous observability/stabilization inequalities) of interest in control theory, modulo - possibly - interior lower-order terms. At any rate, it is for the purpose of establishing the "Carleman estimates, first version," that the energy method in the Riemannian metric plays its critical, dominant role. Such 'first version' is a recovery estimate of a time slice of energy, in terms of observations over the entire time interval.

(2) A refinement or improvement of the Carleman estimates, first version, is called here "Carleman estimates, second version," still without imposing B.C.: it is obtained from the first version by a more routine (but, in the case of the Schrodinger equation, non-trivial) energy method argument, which relies on well-posedness of the dynamics and, ultimately, on the Gronwall's inequality. This step may require an additional structural assumption, as well as additional regularity assumptions, on the energy level terms (e.g., in the case of a general Schrödinger equation written as in Eqn. (8.1), that the real part of the coefficient of the energy level $H^{1}(\Omega)$ terms contained in $F$ in (8.3) be the gradient of a scalar function). Some sort of additional structural requirement is, in fact, intrinsic to the equation to obtain 'well-posedness' [Ho.3], [Miz.1].

(3) Finally, by imposing the B.C. on the second version of the Carleman estimates, we ultimately obtain the sought-after continuous observability/stabilization inequalities (0.3), (0.5) of control theory, modulopossibly - interior lower-order terms.

The interior lower-order terms occur in the Carleman estimates - hence in the C.O.I. and U.S.I. - that are obtained by the Riemannian energy methods of Section 6 (second-order hyperbolic equations), Section 8 (Schrödinger equations), and Section 10 (plate-like equations). However, the more technical pointwise energy methods of Section 7 (second-order hyperbolic equations) and Section 9 (Schrödinger equations, presently in the Euclidean case) lead to pointwise Carleman estimates with the right sign on the interior lower-order term, which accordingly can be dropped. Hence the final Carleman estimates and corresponding C.O.I. and U.S.I. do not contain lower-order term, a big advantage. In particular, they yield global uniqueness theorems for over-determined problem (see Theorems 7.3 and 7.4).

Relaxed version (H.1') of Main assumption. In cases where the 'energy level' terms are not present in any of the aforementioned classes of equations, it is possible to further relax the Main assumption (H.1) = (3.1.1), with the following slightly more general version. Let $(M, g)$ be a compact, finite dimensional Riemannian manifold. Let $\Omega$ be an open bounded, connected subset of $M$. We assume that there exists a vector 
field $H$ such that the covariant differential $D H$ of $H$ (a 2-covariant tensor) satisfies

$$
D H(X, X) \equiv\left\langle D_{X} H, X\right\rangle_{g} \geq 2 \rho|X|^{2}, \quad \forall x \in \Omega, \quad \forall X \in M_{x},
$$

for some constant $\rho>0$, where $D_{X} H$ is the covariant derivative of $H$ w.r.t. $X$.

If $(\mathrm{H} .1)=(3.1 .1)$ is satisfied, with the function $v$, then the vector field $H$ defined by $H \equiv D v$ satisfies $\left(H .1^{\prime}\right)=(3.1 .2)$. On the other hand, $\left(H .1^{\prime}\right)$ $=(3.1 .2)$ may be satisfied with a vector field $H$, which need not be realized as the covariant differential of any (potential) function $v$, satisfying $(\mathrm{H} .1)=$ (3.1.1) [Y.1]. Assumption (3.1.2) was introduced in [Y.1] and in [L-T-Y.3].

An important case where the energy level terms are not present in the equation - and hence $\left(\right.$ H.1 $\left.{ }^{\prime}\right)=(3.1 .2)$ would replace $($ H.1 $)=(3.1 .1)$ is the uniform stabilization problem, where the free dynamics is naturally assumed energy-preserving, as explained in Section 0. This is treated in Theorem 6.5.5.

3.2. Use of assumption (H.1). General statement of Carleman estimates and overview. We provide here an overview of the 'energy method' which encompasses in just one main statement the basic Carleman estimates across all aforementioned single PDE classes of evolution equations, though this common statement corresponds to three separate, different proofs, one proof for each single PDE class. In Part III, we shall give more specific details proper to each single class. However, certain common features can be singled out which are shared by all these single PDE equations. This is achieved in the statement of Theorem 3.2.1 below.

Assume (H.1) $=$ (3.1.1). The pseudo-convex function $\phi(x, t)$. Then define the function $\phi: \Omega \times \mathbb{R} \rightarrow \mathbb{R}$ by

$$
\phi(x, t)=v(x)-c\left(t-\frac{T}{2}\right)^{2},
$$

where $v(x) \geq 0$ is the function assumed in $(\mathrm{H} .1)=(3.1 .1)$. In (3.2.1), the constants $c$ and $T$ are chosen according to the particular evolution equation at hand, as described below.

(1) If the evolution equation is a second-order hyperbolic equation, such as (6.1.1) below, then in (3.2.1) we may choose $c$ and $T$ as follows:

$$
0<c<\rho ; \quad T>T_{0}=2\left(\frac{\max _{x \in \bar{\Omega}} v(x)}{\rho}\right)^{\frac{1}{2}},
$$

$\rho$ as in (3.1.1). The fact that $T$ will have to be 'sufficiently large' is in line with the property that a second-order hyperbolic equation has a finite speed of propagation.

(2) If the evolution equation is either a Schrödinger equation such as (8.1) below, or else a plate-like equation of Euler-Bernoulli type, such as (10.1) below, then we may choose $T$ and $c$ in (3.2.1) as follows: 
$T>0$ arbitrary; $c=c_{T}$ sufficiently large, such that $c T^{2}>4 \sup _{x \in \Omega} v(x)+4 \delta$,

for some small $\delta>0$. The fact that $T$ can be arbitrarily small for Schrödinger and Euler-Bernoulli plates is to be expected, since in both cases there is no finite speed of propagation.

Properties of $\phi(x, t)$. The function $\phi(x, t)$ in (3.2.1) has two key properties, which are exploited in the proofs of the Carleman estimate, first version, in Sections 6-10. They are:

(i) there exists a suitably small constant $\delta>0$, the one in (3.2.3), such that

$$
\phi(x, 0)<-\delta \text { and } \phi(x, T)<-\delta \text {, uniformly in } x \in \Omega ;
$$

(ii) there exist $t_{0}$ and $t_{1}$, with $0<t_{0}<\frac{T}{2}<t_{1}<T$, such that

$$
\min _{x \in \bar{\Omega}, t \in\left[t_{0}, t_{1}\right]} \phi(x, t) \geq-\frac{\delta}{2} .
$$

Step 1. General statement of Carleman estimates, first version. Denote by $E(t)$ the 'natural (mathematical) energy' associated with the specific evolution equation at hand. This will be made more precise for each evolution equation under consideration, in Part III below, Sections 6-10: see specifically Eqn. (6.1.2) (second-order hyperbolic equations), Eqn. (8.2) (Schrödinger equations); Eqn. (10.2) (plate-like equations). Then, one may give the following general statement (to be made more specific and precise for each evolution under consideration, in Part III below), which is obtained by an energy method approach in the Riemannian metric, an ad-hoc method for each specific evolution equation [L-T-Y.1-2], [L-T-Y.4], [T-Y.1].

THEOREM 3.2.1. For each evolution equation such as: (i) the secondorder hyperbolic equation (6.1.1); (ii) the Schrödinger equation (8.1); (iii) the plate-like equation of Euler-Bernoulli type (10.1), denote by $E(t)$ its natural (mathematical) energy [to be given by (6.1.2), (8.2), (10.2), respectively]. Assume the main hypothesis (H.1) = (3.1.1). Let then $\phi(x, t)$ be the function defined in (3.2.1), with the choice of $c$ and $T$ explained above in (3.2.2), (3.2.3). Then: for all values of the parameter $\tau>0$ sufficiently large, say $\tau \geq$ some $\tau_{0}>0$, the following one-parameter family of Carleman estimates holds true for sufficiently smooth solutions $w(t, x)$ :

$$
\begin{aligned}
B_{\Sigma}(w) & +\frac{C_{T}}{\tau} \int_{Q} e^{\tau \phi}|f|^{2} d Q+\operatorname{lot}(w) \\
& \geq\left(p-\frac{c_{T}}{\tau}\right) e^{-\frac{\tau \delta}{2}} \int_{t_{0}}^{t_{1}} E(t) d t-C \tau e^{-\delta \tau}[E(T)+E(0)],
\end{aligned}
$$

where: (i) $p=\rho-c>0$ for second-order hyperbolic equations, where $\rho$ is the constant in assumption (H.1) $=$ (3.1.1), and $c$ is the constant in (3.2.1) or 
(3.2.2); while $p=\rho>0$ for both Schrödinger equations and plate-like equations, where $\rho$ is again defined in (3.1.1) [see Eqns. (6.4.2b), (8.6), (10.6), respectively; see also (7.12b) and (9.10) without l.o.t.]; (ii) $B_{\Sigma}(w)$ are boundary terms defined on $\Sigma$ which can be explicitly obtained for each class of evolution equations under consideration, see Part III (Eqns. (6.4.3), (7.9), (8.7), (10.7)-(10.9), respectively); (iii) finally, lot(w) are interior lower-order terms (below the energy level of $E(t)$, topologically). They may occur, as in the approaches of Section 6 (see Eqn. (6.4.2a)), or Section 8 (see Eqn. (8.5)), or Section 10 (see Eqn. (10.5)); or they may not occur, as in the more technically demanding approaches of Section 7 or Section 9. We elaborate further on this issue in Remark 3.2.2 below.

Remark 3.2.1. We point out explicitly that, in effect, there are three different proofs hidden behind the common statement of Theorem 3.2.1: one for each single class of PDEs mentioned there [L-T-Y.1-2], [T-Y.1], [L-T-Y.4]. Though they share a common philosophical strategy, they are technically different: in particular, they employ different "multipliers." The selection of these multipliers, as well as their use, are tuned to each single class of PDE. See Part III below: Remark 6.4.1 (Second-order hyperbolic equations); Remark 8.1 (Schrödinger equation); Remark 10.1 (plate-like equations). It is, however, an advantage of the "energy method" in the Riemannian metric that it leads to final results for each of the various PDE single classes, which can be expressed by a common, shared statement, where the energy $E(t)$ and the boundary terms $B_{\Sigma}(w)$ depend, however, on the single class, as explicitly given in Part III.

We also note that, as pointed out above, if $v$ is a $g$-strictly convex function on $\bar{\Omega}$, in the sense of hypothesis $($ H.1 $)=(3.1 .1)$, then the function $\phi(x, t)$ in $(3.2 .1)$ is pseudo-convex for some constant $c$. Thus, in principle, the pseudo-differential work of Tataru [Ta.1-3] could be invoked. This is not the case, instead, for coercive vector fields $H$ satisfying the relaxed assumption $\left(\mathrm{H} .1^{\prime}\right)=(3.1 .2)$, which are not the covariant differential of a scalar function (i.e., 'conservative'). Many examples can be given, of course, even in the Euclidean setting, of coercive vector fields that are not conservative. For one example in the Riemannian setting, see [Y.1, Example 3.4]. Thus, the infusion of Riemannian geometric ideas and machinery does help also in the explicit construction, or verifiability, of pseudo-convex functions, as well as in the construction of counter-examples to exact controllability (see Section 5.1: when $\Omega$ has a closed geodesic in its interior). On the other hand, at the pseudo-differential level, the 'right' multiplier for a general evaluation equation is given by the derivative in the dual variable of the principal symbol [Hor.1-2], whereby then only one unifying pseudodifferential proof may be given [Ta.1-3] of a statement such as Theorem 3.2.1. The Riemannian geometric proofs seem to us more friendly.

Step 2. General statement of Carleman estimates, second version. For this step, we need - possibly, but not always - further (mild) restrictions on the structure of the 'energy level terms.' These must be 
such as to guarantee that the following energy inequality holds true: for all $0 \leq s \leq t \leq T$, there exists $C_{T}>0$ such that

$$
|E(t)-E(s)| \leq C_{T}\left[\int_{s}^{t} E(\sigma) d \sigma+G(T)\right],
$$

where

$$
G(T)=B_{\Sigma}(w)+B_{\Sigma, e}(w)+\int_{0}^{T}\|f\|_{H^{\sigma}(\Omega)}^{2} d t+\operatorname{lot}(w)
$$

In (3.2.8), $B_{\Sigma}(w)$ are the boundary terms occurring in (3.2.6); while $B_{\Sigma, e}(w)$ includes those 'boundary terms' that are generated by the energy method (the subscript ' $e$ ' stands for energy), which is employed in seeking to establish inequality (3.2.7).

Comments on the validity of assumption (3.27) for various PDE-classes.

(i) For second-order hyperbolic equations, this energy method consists in multiplying the evolution equation (6.1.1) by $w_{t}$ and integrating by parts. In this case, (3.2.7) holds true with $\sigma=0$ in (3.2.8), $B_{\Sigma, e}(w) \equiv 0$, and with no new restriction imposed on the 'energy level' terms $F(w)$ in (6.1.1).

(ii) For Schrödinger equations, this method consists in multiplying the evolution equation (8.1), e.g., by $\bar{w}_{t}$ [Tr.2], [T-Y.1], or else by $[i \Delta \bar{w}-i \bar{w}]$ [L-T-Z.2] and integrating by parts. In this case, in order to establish (3.2.7) with $\sigma=1$ in (3.2.8), with $B T_{e}(w) \equiv 0$, it is necessary to impose that the energy level terms $F(w)$ in (8.1) be of the type $F(w)=\langle P(t, x), D w\rangle_{g}+r w$ with $\operatorname{Re} P(t, x)=D \chi(t, x)$ for some real function $\chi(t, x)$ [L-T-Z.2]. This (mild) restriction is intrinsic [Hor.3], [Miz.1] due to well-posedness, or lack thereof.

(iii) For plate-like equations of Euler-Bernoulli type (non-hyperbolic), this method consists in multiplying the evolution equation (10.1) by $\Delta w_{t}$ and integrating by parts. This procedure may or may not succeed in establishing inequality (3.2.7) with $\sigma=1$ in (3.2.8), depending on the structure of the energy level term $F(w)$ in (10.1). In particular:

$\left(\mathrm{iii}_{1}\right)$ If $F(w)$ contains, at most, only second-order differential operators on $w$, and zero-order operators on $w_{t}$ [that is, if $F(w)$ has terms one unit below the energy level], then inequality (3.2.7) with $\sigma=1$ in (3.2.8), is satisfied with no further assumptions on $F(w)$.

(iii ${ }_{2}$ ) Energy level terms such as $\frac{\partial}{\partial x} w_{t}, \frac{\partial}{\partial y} w_{t}$, say on $\Omega=\{(x, y)$ : $\left.c \leq y \leq d, h_{1}(y) \leq x \leq h_{2}(y)\right\}$ are acceptable for $F$ to satisfy assumption (3.2.7) [L-T-Y.4, Remark 2.1].

Generally, if $F(w)$ is precisely at the energy-level, some further structural restrictions are needed. These are intrinsic, for otherwise examples are known [Hor.3] where inequality (3.2.7) fails to hold true.

ThEOREM 3.2.2. Assume the setting of Theorem 3.2.1. In addition, assume the validity of inequality (3.2.7). Then: for all values of the pa- 
rameter $\tau>0$ sufficiently large, say $\tau \geq$ some $\tau_{0}>0$, the following oneparameter family of Carleman estimates holds true for sufficiently smooth solutions $w(t, x)$ :

$$
\begin{aligned}
& c_{\phi} B_{\Sigma}(w)+C_{T}\left(t_{1}-t_{0}\right)\left\{B_{\Sigma, e}(w)+\int_{0}^{T}\|f\|_{H^{\sigma}(\Omega)}^{2} d t\right\} \\
& +\operatorname{lot}(w)+\frac{2}{\tau} \int_{Q} e^{\tau \phi}|f|^{2} d Q \geq k_{\phi, \tau_{0}}[E(T)+E(0)],
\end{aligned}
$$

where $k_{\phi, \tau_{0}}>0$, and $B_{\Sigma}(w)$ [and $B_{\Sigma, e}(w)$ ] are explicitly obtained for each class of evolution equations under consideration. The same comments on the occurrence or not of the lot $(w)$ made in point (iii) in the statement of Theorem 3.2.1 apply now, for which again we refer to Remark 3.2.2 below. More details are given in Part III.

Step 3. Continuous observability/stabilization estimates. (Explicit portion of the boundary involved.) Up to Theorem 3.2.2, no use of B.C. is made. Once Theorem 3.2.2 is available, one obtains the sought-after continuous observability/stabilization inequality $(0.3),(0.5)$ by using the corresponding boundary conditions: that is, homogeneous B.C. in the first case, dissipative B.C. in the second case. Thus, the B.C. are used at the very end of the described procedure. It is in this step that the issue arises of how large should the controlled (or observed) or stabilized portion of the boundary be in the final estimates (0.3) and (0.5). To illustrate, the portion $\Gamma_{0}$ of the boundary where the geometric condition

$$
\langle D v, n\rangle \leq 0 \quad \text { on } \Gamma_{0}, \quad D v=\nabla_{g} v
$$

holds can generally be taken as uncontrolled (or unobserved) when zero Dirichlet B.C. are here imposed, so that, generally, the controlled (observed) portion of the boundary may be taken to be $\Gamma_{1}=\Gamma / \Gamma_{0}$. Here $n$ is the outward normal field to $\partial \Omega$ on $M$, and $D$ is the Levi-Civita connection of Section 3.1. This result is illustrated by Theorem 6.5.1 (Dirichlet control) and Theorem 6.5.2 (Neumann control) in the case of second-order hyperbolic equations of Section 6 ; by Theorem 8.3 (Dirichlet control) and Theorem 8.4 (Neuman control) in the case of Schrödinger equations of Section 8; and by Theorem 10.3 in the case of plate-like equations of Section 10. However, again, the situation is geometrically more delicate in dealing with the purely Neumann B.C. say for second-order hyperbolic equations as described in Section 7 below, or Schrödinger equations, as described in Section 9 below. At any rate, this approach yields an explicit portion of the boundary which need not be optimal ('minimal') in all cases. However, the control problem admits a solution - in the sense that C.O.I. (0.3) and the U.S.I. (0.5) hold true as soon as assumption (H.1) $=(3.1 .1)$ is satisfied, perhaps at the price of taking more controlled boundary than it is strictly required. See Section 6.7 below, in particular, Example \#1 there. We elaborate this important point further in the next paragraph. 
Contrast between constant coefficient models versus variable coefficient models in the solution of the control problems. We focus, for definiteness, on second-order hyperbolic equations. Then, for this class, the exact controllability property is always achieved with a sufficiently 'large' controlled portion of the boundary, in particular with control applied over the entire boundary, in the following two cases:

(i) in the case of, say, the canonical wave equation in $\Omega \subset \mathbb{R}^{n}$ (with the Euclidean Laplacian), or even the more general Eqn. (6.3.3) with $\Delta=-\mathcal{A}$, for any dimension $n \geq 1$ [L-T.17],

(ii) the case of the wave equation with variable coefficients, this time, however, in $\operatorname{dim} \Omega=1$.

By contrast, the wave equation with variable coefficients in $\operatorname{dim} \Omega \geq 2$ may very well be non-controllable (see Section 5.1), even if control is applied to the entire boundary $\partial \Omega$. This occurs whenever the Riemannian metric $g$ generated by the coefficients $a_{i j}(x)$ as described in Section 6.3, Eqns. (6.3.2) possesses a closed geodesic in $\mathbb{R}^{n}, n \geq 2$. Then, taking a bounded domain $\Omega$ in $\mathbb{R}^{n}$ which contains such closed geodesic in its interior, yields a noncontrollable system, even if the control is applied to all of $\partial \Omega$ [Ra.1], [Lit.1], [B-L-R.1].

In view of the situation described above, we see that the assumption $($ H.1 $)=(3.1 .1)$ on the strictly convex function excludes the danger that the equation - be it hyperbolic or not-be non-controllable. Thus, we consider the "minimal portion of the boundary" issue as subordinated in importance to the issue of being able to solve the control problem in the first place. Thus, in this viewpoint, the sufficient condition $(\mathrm{H} .1)=(3.1 .1)$ [or its weakened version $\left(\right.$ H. $\left.1^{\prime}\right)=(3.1 .2)$, when the energy level terms are not present] is the foundation of this energy method approach in the Riemannian metric.

Geometric conditions. The continuous observability/stabilization inequalities - C.O.I. and U.S.I. in (0.3) and (0.5) of Section 0, respectively(equivalently, the exact controllability/uniform stabilization results) which we shall present in this paper in Part III, do not require geometrical conditions on the observed (equivalently, controlled) or stabilized portion of the boundary. This feature is in contrast with the great majority of the works on this topic, even those dealing with canonical cases of equations with constant coefficients and no energy level terms. In all these works, geometrical restrictions were imposed that were inherited by the techniques of proof based solely on "classical differential multipliers": see Remark 6.4.1. By contrast, additional tools are needed, such as Lemma 6.5.3, to dispense with unnecessary geometrical conditions - and these tools involve pseudodifferential operators and microlocal analysis: see [L-T.12] for second-order hyperbolic equations, [L-T.13] for plate-like equations, and their critical generalizations and impact in Part IV, dealing with the stabilization of a shallow shell. In particular, Parts III and IV show that differential geometric energy methods combine well with microlocal/pseudo-differential 
operators techniques and lead to the elimination of such (restrictive) geometrical conditions.

Remark 3.2.2. The energy method approach in the Riemannian metric - to be expounded below in Part III - will have two versions. The first version - which may be viewed as a far-reaching generalization of the classical energy methods (multipliers) of the '80s for canonical models (constant coefficients, no energy level terms) as explained in Section 0-yields ultimately a Carleman estimate, first version, such as (3.2.6), in integral form, which contains the lower-order term $\operatorname{lot}(w)$. More specifically, in this first approach, it turns out that the lower-order term has the 'wrong' sign in the estimate, and cannot be dropped. The presence of a $\operatorname{lot}(w)$ which pollutes the desired final estimates is undesirable, since removal of the $\operatorname{lot}(w)$ proceeds by absorbing this term by the appropriate boundary term in the final estimate, via - typically - a compactness/uniqueness argument. This procedure has two disadvantages:

(i) to succeed, it requires appeal to a global unique continuation result under over-determined boundary conditions over the time $T$ of the estimate (identified in $(3.2 .2),(3.2 .3))$. This may be a problem for timedependent $L_{\infty}(Q)$-energy level terms $F(w)$, as assumed [Hor.2], [Hor.5], [I.1-5], [Ta.4-8]. If the coefficients of $F(w)$ are time independent, one converts the uniqueness requirement for the evolution equation to a uniqueness requirement for the corresponding elliptic problems, for which very general results are available [Hor.1], [Hor.2, p. 14].

(ii) When the procedure succeeds, it loses control of the constant in the estimate, as the aforementioned compactness-uniqueness argument is by contradiction.

Thus, it is desirable to avoid the pollution of $\operatorname{lot}(w)$ in the Carleman estimates. This can be done through a more complicated energy method, as we now describe. Indeed, a second version of the energy method approach may be given, which is more general, as its proof includes an additional degree of freedom: this yields a pointwise Carleman estimate (at each time $t$ and point $x$ ), which this time has the 'right' sign $\left[\beta \tau^{3}+\mathcal{O}\left(\tau^{2}\right)\right]$, for $\tau$ large, with $\beta>0$, in front of the lower-order term, as least on an explicitly identified set in time and space [L-T-Z.1]. See Section 7 in the hyperbolic case and Section 9 for Schrödinger equations. Thus, in this case, after some further analysis given in Section 7, the lower-order term may be dropped in the final estimate. Thus, one then obtains, ultimately, a Carleman estimate such as (3.2.6) or (3.2.9), without, however, the presence of the lowerorder term $\operatorname{lot}(w)$. See Theorem 7.2. Consequently, one obtains in one shot new (?) global uniqueness results, Theorems 7.3 and 7.4, as well as observability stabilization estimates such as (0.3) and (0.5). The global uniqueness is precisely in the form needed for the control inequalities in the Neumann case: see Theorem 6.5.6(iii).

Through the PDE control theory literature, the sought-after controltheoretic inequalities - C.O.I. and U.S.I. - have always been given first pol- 
luted by lower-order terms. This includes [B-L-R.1], [Lit.1], [L-T-Y.1] and all 'classical' multipliers literature [Li.1], [K.1]. Few exceptions were [KK.1] (second-order equations with constant coefficient principal part, in line with Russian literature [L-R-S.1]) and the very technical paper [Ta.7]. Our Riemannian approach is inspired by [L-R-S.1, Lemma 1, p. 124] and encompasses [L-T-Z.1], [T-Y.2] for Section 7 below (second-order hyperbolic equations) and Section 9 (Schrödinger equations [L-T-Z.2], along with Remark 10.3 for a purely Euler-Bernoulli plate with hinged B.C. (obtained as an iteration of two Schrödinger's problems).

4. Survey of geometric results and methods for the construction of strictly convex functions and coercive fields.

4.1. The need for a geometrical perspective. Many PDE problems of interest, including higher-order problems, may be expressed in terms of a second-order elliptic operator whose principal part is of the form

$$
\mathcal{A} u \equiv-\sum_{i, j=1}^{n} \frac{\partial}{\partial x_{i}}\left(a_{i j}(x) \frac{\partial u}{\partial x_{j}}\right),
$$

as in (2.2.2). Ellipticity means that

$$
\lambda|\xi|^{2} \leq \sum_{i, j=1}^{n} a_{i j}(x) \xi_{i} \xi_{j} \leq \frac{1}{\lambda}|\xi|^{2}
$$

for some $\lambda>0$. It is of great merit to understand the properties of an operator such as $\mathcal{A}$ in terms which are not based on a specific system of coordinates.

For example, the constant $\lambda$ in (4.1.2) can often be brought closer to 1 by merely introducing an appropriate change of coordinates (in dimension $n=2, \lambda \equiv 1$ may be achieved in simply-connected $\Omega$ by multiplying $\mathcal{A}$ by a positive function and using Morrey's theorem on conformal mapping). The most interesting properties of the operator $\mathcal{A}$ have not been changed by the change of coordinates. This tells us that the constant $\lambda \in(0,1)$ is not an interesting invariant of the operator, even though its existence for any system of coordinates is essential.

What are the invariants of the operator $\mathcal{A}$ ? Which curves, which realvalued functions, etc., play special roles? Such questions may be addressed by introducing the Riemannian metric (see also Section 6.3 )

$$
g=\sum_{i, j=1}^{n} g_{i j}(x) d x_{i} d x_{j},
$$

where $\left(g_{i j}(x)\right)=\left(a_{i j}(x)\right)^{-1}$ for each $x \in \Omega$. Then $\mathcal{A}$ becomes the LaplaceBeltrami operator of $g$, modulo lower-order terms; see (6.3.6). Bicharacteristics $c:[0, T] \rightarrow T^{*} \Omega$ of the wave equation $\frac{\partial^{2}}{\partial t^{2}} u+\mathcal{A} u=0$ are determined by geodesics $\gamma:[0, T] \rightarrow \Omega$ of the Riemannian metric $g$ (Duff [Du.1, 
p. 209], Courant-Hilbert [C-H.1, p. 565]). The conormal vector becomes the $g$-normal vector. Pseudoconvex functions become $g$-convex functions. And the pointwise invariants of $\mathcal{A}$ are the sectional curvatures of the Riemannian metric. For the Riemannian connection $\nabla$, sectional curvature, etc. see e.g. [DoC.1].

4.1.1. Euclidean Space. In Euclidean $\mathbb{R}^{n}$, a familiar and useful function is $\rho_{0}(x)=|x|$, the distance from the origin. It is well known, and widely used, that $f(x) \equiv \frac{1}{2} \rho_{0}(x)^{2}$ is strictly convex. In fact, the Hessian $D^{2} f$ of $f$ is equal to the identity matrix at each point, which is a much stronger property than convexity. We would like to extend the usefulness of this function to non-Euclidean spaces, so that equations with coefficients depending on the space variables may be treated, as emphasized in the Introduction.

In an $n$-dimensional Riemannian manifold $M$, the distance function $\rho$ from any closed subset $\Sigma_{0}$ of $M$ shares some, but not all, of the special properties of the Euclidean distance $\rho_{0}$ from a point. The distance function is defined as

$$
\rho(x) \equiv \inf \left\{L(\sigma) \mid \sigma:[0,1] \rightarrow M, \sigma(0) \in \Sigma_{0}, \sigma(1)=x\right\},
$$

where $L(\sigma)$ denotes the length of a $C^{1}$ or Lipschitz curve $\sigma$. One special property which continues to hold in this more general case is that $|\nabla \rho| \equiv 1$, wherever $\rho$ is smooth. At points where $\rho$ fails to be differentiable (cut points of $\Sigma_{0}$ ), the distributional inequality $|\nabla \rho| \leq 1$ is valid. However, it might not be true that $\frac{1}{2} \rho(x)^{2}$ is strictly convex. This property depends on the curvature of $M$ and on the geometry of $\Sigma_{0}$. A valuable tool in this regard is the matrix Riccati equation (4.3.1), below. For the case $\Sigma_{0}=\left\{p_{0}\right\}$, we have

4.1.2. The Hessian Comparison Theorem. Let $M$ and $\bar{M}$ be two $n$-dimensional Riemannian manifolds, and choose points $p_{0} \in M, \overline{p_{0}} \in \bar{M}$. Choose an orthonormal basis $\left\{E_{1}(0), \ldots, E_{n}(0)\right\}$ for the tangent space to $M$ at $p_{0}$, and similarly an orthonormal basis $\left\{\bar{E}_{1}(0), \ldots, \bar{E}_{n}(0)\right\}$ for the tangent space to $\bar{M}$ at $\bar{p}_{0}$. Let $\gamma$ be the unit-speed geodesic of $M$ with initial conditions $\gamma(0)=p_{0}, \gamma^{\prime}(0)=E_{n}(0)$, and similarly let $\bar{\gamma}$ be the geodesic of $\bar{M}$ with initial conditions $\bar{\gamma}(0)=\bar{p}_{0}, \bar{\gamma}^{\prime}(0)=\bar{E}_{n}(0)$. Extend $E_{i}(0)$ as a parallel vector field $E_{i}(t)$ along $\gamma$, and similarly extend $\bar{E}_{i}(0)$ as a parallel vector field $\bar{E}_{i}(t)$ along $\bar{\gamma}, i=1, \ldots, n$. Write $\rho(x)=d_{M}\left(x, p_{0}\right)$ for the Riemannian distance $d_{M}\left(x, p_{0}\right)$ and $\bar{\rho}(y)=d_{\bar{M}}\left(y, \bar{p}_{0}\right)$.

THEOREM 4.1.1. [S-Y, p. 4] If for all $0 \leq t \leq T$, for all tangent vectors $V$ to $M$ at $\gamma(t)$, and all tangent vectors $\bar{V}$ to $\bar{M}$ at $\bar{\gamma}(t)$, the sectional curvatures

$$
K_{\sigma}^{M} \leq K_{\bar{\sigma}}^{\bar{M}}
$$

where $\sigma$ is spanned by $\gamma^{\prime}(t)$ and $V$, and where $\bar{\sigma}$ is similarly spanned by $\bar{\gamma}^{\prime}(t)$ and $\bar{V}$, then for all $t \in[0, T]$, the Hessian tensors applied to unit tangent 
vectors $V \in T_{\gamma(t)} M$ orthogonal to $\gamma^{\prime}(t)$ and $\bar{V} \in T_{\bar{\gamma}(t)} \bar{M}$ orthogonal to $\bar{\gamma}^{\prime}(t)$ satisfy

$$
D^{2} \rho(V, V) \equiv \nabla_{V, V}^{2} \rho \geq \bar{\nabla}_{\bar{V}}^{2}, \bar{V} \bar{\rho} \equiv \bar{D}^{2} \bar{\rho}(\bar{V}, \bar{V}) .
$$

The proof of the Hessian Comparison Theorem 4.1.1 involves ideas closely connected with H. Rauch's 1951 Comparison Theorem [B-C, p. 250]. We shall indicate a proof based on the matrix Riccati equation below.

COROLlary 4.1.2. If $M$ has all sectional curvatures $K_{\sigma}^{M} \leq b^{2}$, resp. $\leq-\beta^{2}$, for some constant $b$ resp. $\beta$, then for all tangent vectors $V$ at points where $\rho$ is smooth,

$$
D^{2} \rho(V, V) \equiv \nabla_{V, V}^{2} \rho \geq b \cot b \rho\left(\langle V, V\rangle-\langle V, \nabla \rho\rangle^{2}\right)
$$

respectively

$$
\beta \operatorname{coth} \beta \rho\left(\langle V, V\rangle-\langle V, \nabla \rho\rangle^{2}\right) .
$$

COROLLARY 4.1.3. If $M$ is complete and simply connected, and has nonpositive sectional curvatures, then $f(x) \equiv \frac{1}{2} \rho(x)^{2}$ has Hessian greater than or equal to the identity, and is thus uniformly convex.

COROLlary 4.1.4. If $M$ has sectional curvatures $\leq b^{2}$, then for any $r_{0}<\frac{\pi}{2 b}, f(x) \equiv \log \sec b \rho(x)$ has Hessian greater than or equal to the identity, and is thus uniformly convex, on the subdomain where $\rho(x) \leq r_{0}$ and $\rho$ is smooth.

4.2. Functions with convex level sets. This section has connections with pseudo-differential literature [Hor.1-2], [I.1-6], [Ta.1-8].

To ensure the existence of a convex function $v$ on a given Riemannian manifold with boundary $M$, it is roughly sufficient to find a real-valued function $f$ with convex level sets. This fact is well known, but should be kept in mind in the context of establishing boundary controllability. The proof is based on the simple expedient of replacing $f$ with $v(x)=e^{\Lambda f(x)}$ for a sufficiently large constant $\Lambda$. Similar, and sometimes sharper, results may be found by composing $f$ with other functions of one real variable. Convexity of an oriented hypersurface $\Sigma$ is measured by the positivity of its second fundamental form

$$
B_{\Sigma}(X, Y) \equiv\left\langle\nabla_{X} \nu, Y\right\rangle,
$$

where $\nu$ is the unit normal vector of $\Sigma$, and where $X$ and $Y$ are tangent vectors to $\Sigma$. Write $\Sigma_{s}$ for the level set $\{x: f(x)=s\}$, oriented in the direction of increasing values of $s$. A more quantitative statement is as follows [B-G-L.1]:

Lemma 4.2.1. Suppose that $f: M \rightarrow \mathbb{R}$ has convex level sets and nonvanishing gradient, and assume that at points of each level surface $\Sigma_{s}$, for all unit vectors $X$ tangent to $\Sigma_{s}$, that

$$
|\nabla f| B_{\Sigma_{s}}(X, X) \geq c_{1}>0 .
$$


Further let $-c_{2}$ be a lower bound for $\nabla_{\nu, \nu}^{2} f$ and suppose that $|\nabla f|^{2} \geq c_{3}>0$. Write $v(x) \equiv e^{\Lambda f(x)}$.

If $\Lambda$ is chosen large enough that $c_{1}\left(c_{3} \Lambda-c_{2}\right)>c_{2}^{2}$, then for all vectors $Y$,

$$
\nabla_{Y, Y}^{2} v \geq c \Lambda v|Y|^{2},
$$

where $c>0$. Specifically, $0<c<c_{1}$ is small enough so that $\left(c_{3} \Lambda-c_{2}-\right.$ c) $\left(c_{1}-c\right)>c_{2}^{2}$.

In certain interesting examples, $|\nabla f|$ is zero at an isolated minimum of $f$, in a neighborhood of which inequality (4.2.1) nonetheless holds uniformly. In such cases the convexity of $v$ at the minimum point may be obtained by a direct argument [B-G-L.1].

A much more general smoothing theory is due to Greene and $\mathrm{Wu}$ [G-W.1].

Definition 4.2.1. A continuous function $v: M \rightarrow \mathbb{R}$ is uniformly convex if, for some $c>0$, along every geodesic $\gamma$ the function $v(\gamma(s))-c s^{2} / 2$ is convex as a function of arc length $s$ along $\gamma$.

Greene and Wu show [G-W.1, p. 214]:

ThEOREM 4.2.2. Let $v: M \rightarrow \mathbb{R}$ be continuous and uniformly convex in the sense that, for some $c>0$, along every geodesic $\gamma$ the function $v(\gamma(s))-c s^{2} / 2$ is convex as a function of arc length salong $\gamma$. Then $v$ is the uniform limit of smooth functions $v_{\varepsilon}: M \rightarrow \mathbb{R}$ with $\nabla_{Y, Y}^{2} v_{\varepsilon}>(c-\varepsilon)|Y|^{2}$ for all vectors $Y$.

As a consequence, they show that: on any complete, noncompact manifold with positive sectional curvature, there is a smooth, uniformly convex function $v_{\varepsilon}$. For this situation, they make use of the convexity of the continuous function $v(x)=e^{\Lambda \rho(x)}$, where $\rho(x)$ is the limit of $d_{M}(x, \sigma(t))-t$ as $t \rightarrow \infty$, and where $\sigma:[0, \infty) \rightarrow M$ is a unit-speed geodesic which minimizes length between any two of its points. See [G-W.1, pp. 292-6]. It had been shown by Cheeger and Gromoll in $[\mathrm{CG}]$ that the sublevel sets of $\rho$ are totally convex.

4.3. The Matrix Riccati Equation. Consider a smooth real-valued function $\rho: M \rightarrow \mathbb{R}$ on a Riemannian manifold $M$, with the special property that $|\nabla \rho| \equiv 1$. (For the distance function from a set $\Sigma_{0}$, it may be necessary to stay away from any cut points, which form a closed set of measure zero, to achieve smoothness of $\rho$; cf. Section 5.) Then for various real values $s$, the level sets $\Sigma_{s}$ defined by $\rho=s$ form a smooth one-parameter family of hypersurfaces. Write $\nu$ for the vector field $\nabla \rho$, so that $\nu(x)$ is a unit normal vector to the hypersurface $\Sigma_{s}$ passing through $x$. Let $\gamma: \mathbb{R} \rightarrow M$ be an orbit of $\nu$, that is, for each $s \in \mathbb{R}, d \gamma(s) / d s=\nu(\gamma(s))$. Note that after adding a constant to the independent parameter $s$, we may assume that $\gamma(s) \in \Sigma_{s}$ for all $s$. Moreover, the curve $\gamma$ has minimum length between any two of its points. In fact, let two points of the curve 
be $\gamma\left(s_{0}\right)$ and $\gamma\left(s_{1}\right)$. If $\sigma:[0,1] \rightarrow M$ is any curve joining $\gamma\left(s_{0}\right)=\sigma(0)$ to $\gamma\left(s_{1}\right)=\sigma(1)$, then

$$
\left|\frac{d \sigma(t)}{d t}\right| \geq\left\langle\nu, \frac{d \sigma(t)}{d t}\right\rangle=\frac{\partial \rho(\sigma(s))}{\partial s} .
$$

It follows that the length of $\sigma$ is at least $\rho\left(\gamma\left(s_{1}\right)\right)-\rho\left(\gamma\left(s_{0}\right)\right)=s_{1}-s_{0}$, which is the length of $\gamma\left(\left[s_{0}, s_{1}\right]\right)$. In particular, $\gamma$ is a geodesic. Since there is an orbit of $\nu$ passing through each point of $M$, we conclude that $\nabla_{\nu} \nu \equiv 0$.

Now the Hessian of $\rho$ may be written $\nabla_{V, W}^{2} \rho=\left\langle V, \nabla_{W}(\nabla \rho)\right\rangle$ for any tangent vectors $V, W$. In particular, with $V=W=\nu=\nabla \rho$, we find that $\nabla_{\nu, \nu}^{2} \rho=0$; and if $W=\nu,\langle V, \nu\rangle=0$, then $\nabla_{V, \nu}^{2} \rho=\nabla_{\nu, V}^{2} \rho=0$. These properties are exactly as for the Euclidean distance function $\rho_{0}$.

The remaining part of the Hessian of $\rho$ can be interpreted as an $(n-1) \times(n-1)$ matrix $\nabla_{E_{i}(s), E_{j}(s)}^{2} \rho=A_{i j}(s)=B_{\Sigma_{s}}\left(E_{i}(s), E_{j}(s)\right)$. For this purpose, consider a point $x_{0} \in \Sigma_{s_{0}}$ and let $\gamma: \mathbb{R} \rightarrow M$ be the geodesic passing through $x_{0}=\gamma\left(s_{0}\right)$ which is an orbit of $\nu$. Choose an orthonormal basis $E_{1}\left(s_{0}\right), \ldots, E_{n-1}\left(s_{0}\right)$ for the tangent space to $\Sigma_{s_{0}}$ at $x_{0}$. As above, we extend this orthonormal basis as parallel vector fields along $\gamma$, so that for all $s, E_{1}(s), \ldots, E_{n-1}(s)$ is an orthonormal basis for the tangent space to $\Sigma_{s}$ at $\gamma(s)$.

Note that $A_{i j}(s)$ represents both the second fundamental form of $\Sigma_{s}$ and the nontrivial part of the Hessian of $\rho$. In fact, $A_{i j}=\left\langle E_{i}, \nabla_{E_{j}}(\nabla \rho)\right\rangle=$ $B_{\Sigma_{s}}\left(E_{i}, E_{j}\right)=\nabla_{E_{i}, E_{j}}^{2} \rho=A_{j i}, i, j=1, \ldots, n-1$. We may now compute

$$
\frac{\partial}{\partial s} A_{i j}(s)=\nu\left(\left\langle\nabla_{E_{i}} \nu, E_{j}\right\rangle\right)=\left\langle\nabla_{\nu} \nabla_{E_{i}} \nu, E_{j}\right\rangle+\left\langle\nabla_{E_{i}} \nu, \nabla_{\nu} E_{j}\right\rangle,
$$

where the last term vanishes since $E_{j}$ is parallel along $\gamma$. For the same reason, we have the Lie bracket $\left[E_{i}, \nu\right]=\nabla_{E_{i}} \nu=A_{j i} E_{j}$, so that the curvature tensor

$$
\begin{aligned}
R_{i n n j} & =R\left(E_{i}, \nu, \nu, E_{j}\right):=\left\langle\nabla_{\nu} \nabla_{E_{i}} \nu-\nabla_{E_{i}} \nabla_{\nu} \nu+\nabla_{\left[E_{i}, \nu\right]}, E_{j}\right\rangle \\
& =\frac{\partial}{\partial s} A_{i j}(s)-0+A_{i k}(s) A_{k j}(s),
\end{aligned}
$$

since $\nabla_{\nu} \nu \equiv 0$. A more familiar form of this Matrix Riccati Equation is

$$
\frac{\partial}{\partial s} A_{i j}(s)+A_{i k}(s) A_{k j}(s)=-R_{i n j n}(\gamma(s)) .
$$

The Matrix Riccati Equation has importance in many other contexts, such as optimal control [L-T.11], [L-T.24], [R, p. 215].

4.4. Matrix Riccati Comparison Theorem. Although the Matrix Riccati Equation (4.3.1) is nonlinear, it leads to powerful techniques for comparing functions such as the distance functions from given subsets on two manifolds. Namely: 
THEOREM 4.4.1. Suppose that $\Sigma_{0}$ resp. $\bar{\Sigma}_{0}$ are smooth, transversely oriented hypersurfaces of the Riemannian manifolds $M$ resp. $\bar{M}$, and let $\rho: M \rightarrow \mathbb{R}$ resp. $\bar{\rho}: \bar{M} \rightarrow \mathbb{R}$ be the signed distance to a point of $M$ resp. $\bar{M}$ from $\Sigma_{0}$ resp. $\bar{\Sigma}_{0}$. Assume that $\rho$ and $\bar{\rho}$ are smooth. Let $\gamma$ resp. $\bar{\gamma}$ be unit-speed geodesics meeting $\Sigma_{0}$ resp. $\bar{\Sigma}_{0}$ at $\gamma(0)$ resp. $\bar{\gamma}(0)$ with initial tangent vector $\gamma^{\prime}(0)=\nu(\gamma(0))$ resp. $\bar{\gamma}^{\prime}(0)=\bar{\nu}(\bar{\gamma}(0))$, the unit normal vectors to $\Sigma_{0}$ resp. $\bar{\Sigma}_{0}$. Assume that for all $0 \leq s \leq s_{1}$, for all unit vectors $W \in T_{\gamma(s)} M$ orthogonal to $\gamma^{\prime}(s)$, and $\bar{W} \in T_{\bar{\gamma}(s)} \bar{M}$ orthogonal to $\bar{\gamma}^{\prime}(s)$, that the sectional curvatures $R\left(W, \gamma^{\prime}, W, \gamma^{\prime}\right) \leq \bar{R}\left(\bar{W}, \bar{\gamma}^{\prime}, \bar{W}, \bar{\gamma}^{\prime}\right)$. As initial conditions, assume that for all unit vectors $V \in T_{\gamma(0)} \Sigma_{0}$ and for all unit vectors $\bar{V} \in T_{\bar{\gamma}(0)} \bar{\Sigma}_{0}$, the normal curvatures

$$
B_{\Sigma_{0}}(V, V)=\nabla_{V, V}^{2} \rho(\gamma(0)) \geq \bar{\nabla}_{\bar{V}}^{2}, \bar{V} \bar{\rho}(\bar{\gamma}(0))=B_{\bar{\Sigma}_{0}}(\bar{V}, \bar{V}) .
$$

Then for all $0 \leq s \leq s_{1}$, for all unit vectors $W \in T_{\gamma(s)} M$ orthogonal to $\gamma^{\prime}(s)$, and $\bar{W} \in T_{\bar{\gamma}(s)} \bar{M}$ orthogonal to $\bar{\gamma}^{\prime}(s)$, we have

$$
B_{\Sigma_{s}}(W, W)=\nabla_{W, W}^{2} \rho(\gamma(s)) \geq \bar{\nabla}_{\bar{W}}^{2}, \bar{W} \bar{\rho}(\bar{\gamma}(s))=B_{\bar{\Sigma}_{s}}(\bar{W}, \bar{W}) .
$$

Theorem 4.4.1 has a long statement, but a relatively short proof. First note that the hypotheses of Theorem 4.4.1 allow us to interpose a "scalar" second fundamental form $\widetilde{A}_{i j}(0)=\alpha_{0} \delta_{i j}$ between $A_{i j}(0)$ and $\bar{A}_{i j}(0)$ and a curvature tensor of "scalar" form $\widetilde{R}_{i n j n}=\kappa(s) \delta_{i j}$ between $R_{i n j n}$ and $\bar{R}_{i n j n}$. Here $\alpha_{0}$ is chosen less than or equal to the smallest principal curvature of $\Sigma_{0}$ at $\gamma(0)$, but greater than or equal to the largest principal curvature of $\bar{\Sigma}_{0}$ at $\bar{\gamma}(0)$. We write the resulting inequality as $\left(A_{i j}(0)\right) \geq\left(\widetilde{A}_{i j}(0)\right) \geq\left(\bar{A}_{i j}(0)\right)$, where an inequality between symmetric matrices is to be understood in the sense that the difference of the two sides is positive semi-definite. Also, we choose here $\kappa(s)$ less than or equal to the smallest sectional curvature of $\bar{M}$ in plane sections containing $\bar{\gamma}^{\prime}(0)$, but greater than or equal to the largest sectional curvature of $M$ in plane sections containing $\gamma^{\prime}(0)$. We define $\widetilde{A}_{i j}(s):=\alpha(s) \delta_{i j}$, where $\alpha(s)$ is the solution of the scalar Riccati equation

$$
\frac{d \alpha(s)}{d s}+\alpha(s)^{2}=-\kappa(s), \quad 0 \leq s \leq s_{1},
$$

with the initial condition $\alpha(0)=\alpha_{0}$ as already chosen. We shall show that (1) $\left(A_{i j}(s)\right) \geq\left(\widetilde{A}_{i j}(s)\right)$ and $\left.(2)\left(\widetilde{A}_{i j}(s)\right)\right) \geq\left(\bar{A}_{i j}(s)\right)$ for all $0 \leq s \leq s_{1}$. Thus, it will suffice to prove Theorem 4.4.1 in the two special cases where (1) $\left(A_{i j}(s)\right)$ and $\left(R_{i n j n}(\gamma(s))\right)$ are in the scalar form, or where $(2)\left(\bar{A}_{i j}(s)\right)$ and $\left(\bar{R}_{\text {injn }}(\bar{\gamma}(s))\right)$ are in the scalar form. In both cases, after substituting $\widetilde{A}_{i j}$ for one of $A_{i j}$ or $\bar{A}_{i j}$, we have

$$
\sum_{k} A_{i k}(s) \bar{A}_{k j}(s)=\sum_{k} \bar{A}_{i k}(s) A_{k j}(s),
$$


that is, for each $s$, the second fundamental forms $B_{\Sigma_{s}}\left(E_{i}, E_{j}\right)$ and $B_{\bar{\Sigma}_{s}}\left(\bar{E}_{i}\right.$, $\bar{E}_{j}$ ) have a common basis of curvature directions. It remains only to prove inequality 4.4.1 under the additional assumption 4.4.3. In this case, write the difference matrix $D_{i j}(s)=A_{i j}(s)-\bar{A}_{i j}(s)$. Then $\frac{d}{d s} D_{i j}(s)=$ $-A_{i k} A_{k j}+\bar{A}_{i k} \bar{A}_{k j}-R_{i n j n}+\bar{R}_{i n j n}$ by the matrix Riccati equation (4.3.1), so that $\left(\frac{d}{d s} D_{i j}(s)\right) \geq\left(-D_{i k}\left(A_{k j}+\bar{A}_{k j}\right)\right)$ since $\left(R_{i n j n}\right) \leq\left(\bar{R}_{i n j n}\right)$ and using the commutativity (4.4.3). Also, we have the initial inequality $\left(D_{i j}(0)\right) \geq 0$. Let $\lambda(s)$ be the smallest eigenvalue of $\left(D_{i j}(s)\right): \lambda(s)$ is Lipschitz continuous as a function of $s$, and $\lambda(0) \geq 0$. Consider any $s_{0} \in\left(0, s_{1}\right]$ where $d \lambda / d s$ exists. Assume $E_{1}\left(s_{0}\right)$ is chosen to be an eigenvector of $\left(D_{i j}\left(s_{0}\right)\right)$ with eigenvalue $\lambda\left(s_{0}\right)$. Then $D_{11}\left(s_{0}\right)=\lambda\left(s_{0}\right)$ and for all $s, D_{11}(s) \geq \lambda(s)$. Thus

$$
\frac{d \lambda}{d s}\left(s_{0}\right)=\frac{d D_{11}}{d s}\left(s_{0}\right) \geq-D_{1 k}\left(A_{k 1}+\bar{A}_{k 1}\right) \geq-C \lambda\left(s_{0}\right),
$$

where $C:=\sup \left\{B_{\Sigma_{s}}(V, V)+B_{\bar{\Sigma}_{s}}(\bar{V}, \bar{V})\left|0 \leq s \leq s_{1},\right| V|=| \bar{V} \mid=1\right\}$. This implies $\lambda(s) \geq e^{-C s} \lambda(0) \geq 0$ for all $s \in\left[0, s_{1}\right]$. That is, $\left(A_{i j}(s)\right) \geq\left(\bar{A}_{i j}(s)\right)$, which is the conclusion (4.4.1) of Theorem 4.4.1.

As a corollary, we may prove the Hessian Comparison Theorem 4.1.1. We first show that in the case where the reference set $\Sigma_{0}$ is a point $p_{0}$ of $M$, all normal curvatures of $\Sigma_{s}$ equal $1 / s$ plus a term which approaches zero uniformly as $s \rightarrow 0$ (proof, based on normal coordinates at $p_{0}$, omitted here.) Thus, applying the Matrix Riccati Comparison Theorem 4.4.1 on intervals $\left[s_{0}, s_{1}\right]$ with $s_{0}$ close to 0 , we obtain the conclusion of Theorem 4.1.1 within an arbitrarily small tolerance, and hence exactly.

4.5. Nonsmooth distance function. We indicated above that Greene and $\mathrm{Wu}$ were able to convert the distance function from infinity on a complete manifold of positive curvature into a smooth function. But in general, a locally convex distance function may be nonsmooth in a nonconvex way. As an example, we consider the two-dimensional, flat cylinder $S^{1} \times \mathbb{R}$, with coordinates $(\theta, s)$. The distance function $\rho$ from $p_{0}=(0,0)$ fails to be smooth along the cut locus $\{\theta=\pi\}$, although everywhere else, $\frac{1}{2} \rho(x)^{2}$ is strictly convex. In fact, locally near $\{\theta=\pi\}, \frac{1}{2} \rho(x)^{2}$ is the minimum of two locally convex functions, whereas the maximum of two such functions would be convex, in the sense of Definition 4.2 .1 above. In order to obtain a convex function $v(x)=\varphi(\rho(x))$ without discarding the cut locus, we need only to ensure the strict convexity of the level sets $\Sigma_{s}$ of $\rho$ and to choose $\varphi: \mathbb{R} \rightarrow \mathbb{R}$ to be strictly decreasing as a function of the distance $|\rho(x)|$.

As an interesting case, we consider a result of Burago and Zalgaller ([B-Z.1, p. 259]):

THEOREM 4.5.1. Suppose $\Omega$ is a smooth domain in $M$ which has sectional curvatures bounded below by a constant $\kappa$, which lies within a distance $R$ from its boundary, and whose boundary has all normal curva- 
tures greater than a positive constant $\alpha_{0}$. Then there is a convex function $v: \Omega \rightarrow \mathbb{R}$, provided that either $\kappa \geq 0$, or $\alpha_{0} \geq \sqrt{-\kappa} \tanh \sqrt{-\kappa} R$.

A proof of this result follows from the Matrix Riccati Comparison Theorem 4.4.1 and Theorem 4.2.2. In fact, let $\alpha(s),-R \leq s \leq 0$, be the solution of the scalar Riccati equation (4.4.2)

$$
\frac{d \alpha(s)}{d s}+\alpha(s)^{2}=-\kappa,
$$

with the "initial" condition $\alpha(0)=\alpha_{0}$. $(\alpha(s)$ is written as $-Q(f)$ in [B-Z.1].) If $\kappa>0$, write $\kappa=b^{2}$; then $\alpha(s)=b \cot b\left(s-s_{0}\right)$, where $s_{0}<0$ is determined by $b \cot b s_{0}=-\alpha_{0}$. If $\kappa<0$, and we write $\kappa=-\beta^{2}$, then three cases may occur, depending on the magnitude of $\alpha_{0}$ : when $\alpha_{0}>\beta$, we find $\alpha(s)=\beta \operatorname{coth} \beta\left(s-s_{0}\right)$, where $s_{0}<0$ is determined by $\beta \operatorname{coth} \beta s_{0}=-\alpha_{0}$; when $\alpha_{0}=\beta$, we find $\alpha(s) \equiv \beta$; and when $\alpha_{0}<\beta$, we find $\alpha(s)=$ $\beta \tanh \beta\left(s-s_{0}\right)$, where $s_{0}<0$ is determined by $\beta \tanh \beta s_{0}=-\alpha_{0}$. In particular, $\alpha(s)$ remains positive as $s$ decreases from 0 to $-R$ under the hypotheses stated.

We apply the Matrix Riccati Comparison Theorem, with $M$ in the role of $\bar{M}$ and the boundary of $\Omega$ in the role of $\bar{\Sigma}_{0}$. The comparison manifold of constant sectional curvature $\kappa$ plays the role of $M$, and $\Sigma_{0}$ is a hypersurface of constant normal curvatures $\alpha_{0}$. Then the hypersurface $\Sigma_{s}$ at constant signed distance $s$ from $\Sigma_{0}$ is totally umbilic with normal curvatures $\alpha(s)$. Note that the parameter domain $-R \leq s \leq 0$ in our case lies on the opposite side of the initial hypersurface at $s=0$; since the proof follows from a first-order differential inequality, the conclusion will also have the opposite sense from (4.4.1). Thus

$$
B_{\Sigma_{s}}(V, V) \geq \alpha(s)\langle V, V\rangle,
$$

$-R \leq s \leq 0$. Thus, under the hypotheses stated, wherever $\rho(x)$ is smooth, $v(x)=e^{\Lambda \rho(x)}$ will be convex for large enough $\Lambda$.

Finally, near a general point, since $\rho<0$ is locally the maximum of a number of locally smooth functions with convex sublevel sets, it has convex sublevel sets. The continuous convex function $v: M \rightarrow \mathbb{R}$ may now be approximated by smooth, uniformly convex functions $v_{\varepsilon}: M \rightarrow \mathbb{R}$ according to Theorem 4.2.2.

Acknowledgement. The authors wish to thank Yaroslav Kurylev, Loughborough University, UK, for directing their attention to the results of Burago and Zalgaller.

4.6. Construction of a strictly convex function in the 2-d case via curvature flows. The following result is proved by using, among other things, Lemma 4.2.1 as well as a nonlinear parabolic equation which arises in a quite unrelated geometric problem of curve-shortening flows [Gr.1], [C-Z.1]. 
THEOREM 4.6.1. [B-G-L.1] Let $\bar{\Omega}$ be a two-dimensional smooth compact Riemannian surface whose boundary $\partial \Omega$ has positive second fundamental form. Assume there are no closed geodesics in $\Omega$. Then there exists $a C^{2}$-strictly convex function $v$ in $\bar{\Omega}$.

Remark 4.6.1. It is therefore very important to be able to discern whether a given $\Omega$, with a given Riemannian metric, contains closed geodesics. In the 2-d case, [B-G-L.1, Section 5] describes a computational method which implements the parabolic flow (entering the proof of Theorem 4.6.1), and enables one to either find geodesics homotopic to a component of $\partial \Omega$, or else to conclude that there are no closed geodesics.

5. A Riemannian geometric approach for exact controllability of second-order hyperbolic equations. A "view from the boundary" which verifies the geometric optics condition.

5.1. Introduction. Counterexamples. Hypotheses. Main exact controllability statement. Introduction. One of the important advances in the subject of boundary control for (linear) second-order hyperbolic equations has been the almost necessary and sufficient criteria for exact controllability and uniform stabilization involving bicharacteristics [Lit.1], [B-L-R.1]. Indeed, the opinion has sometimes been expressed to the effect that this whole subject of exact controllability/uniform stabilization for (linear) second-order hyperbolic equations has been completely resolved. However, one may counter such a hasty opinion with various considerations. To begin with, the conditions involving bicharacteristics are generally difficult to verify, as these are solutions of a system of nonlinear ordinary differential equations (Hamiltonian). Thus, the need arises for the infusion of other ideas into this subject, not only for expressing tests for exact controllability/stabilization in terms of (perhaps more restrictive, but also) more easily recognizable geometric properties, but also for making contact and building links with other (perhaps unsuspected) mathematical areas. The subsequent connection [Ta.1-3] made with pseudo-differential multipliers (symbols) derived from pseudo-convex functions (defined in terms of double Poisson brackets) is one positive case in point. Though providing only, definitely, sufficient conditions in the case of second-order hyperbolic equations, this method of Tataru has a broader scope, as it applies to general non-hyperbolic evolution equations as well. Another similar case in point - which provides the additional benefits of construction and checkability of assumptions, friendlier proofs, with closer and more recognizable links to 'classical methods' of the ' 80 s - is the energy method in the Riemannian metric outlined in Section 3, of which we shall see more definite details in Part III. Here we shall, instead, present a method that singles out an alternative set of sufficient conditions for exact controllability of secondorder hyperbolic equations, even though the resulting proofs are ultimately based on the original 'bicharacteristic' conditions, and hence in technical results in PDE theory [Hor.1-2], [Tay.1]. The focus of this method to be now 
explained is, for now, on the qualitative relationship between Riemannian geometry and boundary control of second-order hyperbolic PDEs. Thus, the treatment in this section will not attempt to express exact controllability in terms of the optimal choice of Sobolev spaces, or, of portion of the controlled boundary, unlike the energy method of Section 3 (to be detailed in Part III). Nor does the treatment of the present section attempt to find the optimal smoothness of the Riemannian metric and of other coefficients of the hyperbolic equation.

An alternative geometric approach. We shall present another line of investigation - in a sense, even more geometric in flavor - this one valid only for hyperbolic equations. In fact, it will exploit the well-known identification - available exclusively for hyperbolic problems - between the geometric optics notion of bi-characteristics (the carriers of energy) and the Riemannian geometric notion of geodesics with respect to a natural metric defined in terms of the coefficients of the principal part of the elliptic operator in the equation (Duff [Du.1, p. 209], Courant-Hilbert [C-H.1, p. 565]). See Section 6.3 below. This way, Riemannian geometry is merged with geometric optics. Accordingly, in this approach, the focus will be put on the geodesics.

Construction of counterexamples. One advantage is obtained at once, at the level of constructing counterexamples. Riemannian geometry offers plenty of manifolds $(M, g)$ or even $\left(\mathbb{R}^{n}, g\right)$, of dimension at least equal to 2 , which possess a closed geodesic. It then suffices to take any bounded set $\Omega$, properly containing such closed geodesic in its interior, to have an example (in dimension greater or equal to 2) of a hyperbolic equation as in Section 6.3, where exact controllability fails, even if the control action is applied to the entire boundary $\partial \Omega$. Indeed, since the closed geodesic (bicharacteristic) does not touch the boundary, the necessary condition for exact controllability provided by the geometric optics approach [Ra.1], [Lit.1], [B-L-R.1] permits us to conclude that exact controllability is not possible, even if the boundary is fully controlled. This is an instance of the synergistic advantage that derives from marrying the Riemannian geometric viewpoint with the geometric optics approach. Many specific examples of this phenomenon may be given.

Counterexample \#1: See [Y.1, Example 4.1], with $g=(d x d x+$ $d y d y) /\left[\left(1+x^{2}+y^{2}\right)^{2}\right]$.

Counterexample \#2 [G-L.1]: The Frisbee. This example is a large flat $n$-disk surrounded by a moderate-sized region of positive curvatures and a thin region of negative curvature, resembling the inside surface of the flying toy known as the Frisbee (apologies to the Wham-O Corporation).

This is an example with non-constant sectional curvature. We claim that for the Frisbee example, the Riemannian wave equation defined on it is not exactly controllable from the boundary. In fact, the sphere $\left\{r=r_{0}\right\}$ is totally geodesic as a submanifold of $\Omega$, and therefore any of the sphere's own great circles will be closed geodesics of $\Omega$. Any one of these closed geodesics suffices to make boundary controllability impossible, as seen above. 
Counterexample \#3 [G-L.1]: Manifold with a big bulge. Let $\bar{\Omega}$ be a Riemannian manifold-with-boundary, diffeomorphic to the closed ball of $\mathbb{R}^{2}$, which contains in its interior a smooth subdomain $\bar{D}$ isometric to the closed hemisphere of the unit sphere $S^{2} \subset \mathbb{R}^{3}$. We assume that $\partial \Omega$ has positive second fundamental form. Such a manifold may be constructed as a hypersurface of revolution in $\mathbb{R}^{3}$, with a generating curve which begins orthogonally to the axis of revolution with a quarter-circle of radius 1, and ends at a moment when it is traveling away from the axis of rotation. Alternatively, such an example may be constructed by "pasting in" an isometric copy of the closed hemisphere in the interior of a reference manifold whose boundary has positive second fundamental form.

One may show that there exist closed geodesics in the boundary of $D$. Hence, again, the Riemannian wave equation cannot be exactly controllable on such manifold $\Omega$ with a big bulge, even if its entire boundary $\partial \Omega$ is controlled.

The manifold $\bar{\Omega}$ also does not allow any convex functions, as follows from [G-L.1, Proposition 5.3] using a domain slightly larger than $D$.

A view from the boundary. The computational energy method in the Riemannian metric described in Section 3 (and to be further analyzed in Part II) makes its main assumption (H.1) $=(3.1 .1)$ in the interior of $\Omega$. By contrast, the presently described alternative line of research takes a "view from the boundary" as its distinguishing feature.

Hypotheses. This approach is based on three assumptions: (i) that the boundary $\partial \Omega$ has positive second fundamental form: this analytical hypothesis is slightly stronger than the geometric assumption that $\partial \Omega$ be strictly convex (in the Riemannian metric); (ii) that 'chords' be unique; (iii) that chords be nondegenerate.

A definition and analysis of these assumptions will be given in Section $5.2-5.4$ below.

Statement of exact controllability results of the Riemannian wave. Consider a compact, $n$-dimensional Riemannian manifold-withboundary $\bar{\Omega}$. We assume that $\partial \Omega$ is smooth and nonempty, and that the metric of $\bar{\Omega}$ is smooth, i.e., $C^{\infty}$. We are interested in the boundary control of the following natural hyperbolic partial differential equation (Riemannian wave equation) on $\Omega \times[0, T]$ :

$$
\frac{\partial^{2} u}{\partial t^{2}}=\Delta_{g} u=\sum_{i, j=1}^{n} \frac{1}{\gamma} \frac{\partial}{\partial x_{i}}\left(\gamma g^{i j}(x) \frac{\partial u}{\partial x_{j}}\right),
$$

for all $(x, t) \in \Omega \times[0, T]$, where $\left(x_{1}, \ldots, x_{n}\right)$ are arbitrary local coordinates, $g^{i j}(x)$ are the entries of the inverse matrix to the coefficients $g_{i j}(x)$ of the Riemannian metric, and $\gamma$ is the Riemannian volume integrand: $\gamma(x)=\sqrt{\operatorname{det}\left(g_{i j}(x)\right)}$. We consider the problem of the control in time $T$ of equation (5.1.1) from the entire boundary $\partial \Omega$. More precisely, we consider the boundary conditions 


$$
u(x, t)=U(x, t) \text { for all }(x, t) \text { on } \partial \Omega \times[0, T],
$$

where $U \in H^{\frac{1}{2}}(\partial \Omega \times[0, T])$ is the control, i.e., a function which may be chosen as needed. The controllability question is whether, given any initial conditions

$$
u(x, 0)=u_{0}(x), \quad \frac{\partial u}{\partial t}(x, 0)=u_{1}(x),
$$

with finite energy, there is a choice of controls $U \in H^{\frac{1}{2}}(\partial \Omega \times[0, T])$ such that the solution of (5.1.1) with initial conditions (5.1.3) and boundary conditions (5.1.2) vanishes identically on $\Omega \times[T, \infty)$. Equivalently, we ask whether for some choice of controls $U$ the terminal Cauchy values vanish: $u(x, T)=0, \frac{\partial u}{\partial t}(x, T)=0$ for all $x$ in $\Omega$.

In this section we wish to describe two main results (Theorems 5.1.1, 5.1.2). We shall refer to [G-L.1] for complete proofs.

We define a chord to be a curve in $\bar{\Omega}$ of shortest length between two boundary points.

THEOREM 5.1.1. [G-L.1] Suppose that any two boundary points of the manifold $\bar{\Omega}$ are connected by a unique chord, which is nondegenerate. Assume that $\partial \Omega$ has positive second fundamental form. Then the hyperbolic equation (5.1.1) is controllable from $\partial \Omega$ by means of boundary conditions (5.1.2) in any time $T>T_{0}=\operatorname{diam}_{\bar{\Omega}}(\partial \Omega)$.

Here, the diameter of the boundary of $\Omega$ is the maximum distance between any two of its points, with respect to the distance measured in $\bar{\Omega}$, that is: the length of the longest chord of $\bar{\Omega}$. Our convention for the sign of the second fundamental form is such that if $\Omega$ is a ball of radius $r$ in $\mathbb{R}^{n}$, with the Euclidean metric, then $\partial \Omega$ has a positive second fundamental form $B=r^{-1} d s^{2}$.

The "uniqueness" of a chord $\gamma:[0, a] \rightarrow \bar{\Omega}$ is understood modulo reparameterizations $s \mapsto \gamma(A s+B),(A, B \in \mathbb{R})$ of the independent variable $s$.

Remark 5.1.1. The hypothesis of Theorem 5.1.1 that $\partial \Omega$ has positive second fundamental form at each point $p \in \partial \Omega$ may be computed most easily by making a linear change of coordinates so that the coordinate hyperplane $x_{n}=$ const., which passes through $p$ is tangent to $\partial \Omega$ there, so that the $n^{\text {th }}$ coordinate vector is the inward unit normal to $\partial \Omega$ at $p$. Then, in local coordinates, the condition that $\partial \Omega$ has a positive second fundamental form amounts to having a symmetric matrix $\left\{B_{i j}\right\}$, with explicit entries $B_{i j}$ in terms of the Christoffel symbols [G-L.1, p. 150], be positive definite at $p$. The matrix $\left\{B_{i j}\right\}$ represents the second fundamental form of $\partial \Omega$ in these coordinates. Equivalently, if $\bar{\Omega}$ is extended to be a smooth subdomain of a Riemannian manifold $M$, one requires that any geodesic of $M$ which is tangent to $\partial \Omega$ at $p$ remains outside of $\bar{\Omega}$ to second order at $p$. This property has been called "pseudo-convexity" in the PDE literature.

We shall refer to the infimal value $T_{0}$ as the "optimal time of control," even though $T_{0}$ itself may not be a control time. More generally, we shall consider the hyperbolic equation with additional lower-order terms: 


$$
\frac{\partial^{2} u}{\partial t^{2}}=\Delta_{g} u+\sum_{i=1}^{n} V^{i}(x, t) \frac{\partial u}{\partial x_{i}}+a(x, t) u
$$

where $\left(V^{1}, \ldots, V^{n}\right)$ are the components, in any local system of coordinates $x=\left(x_{1}, \ldots, x_{n}\right)$ for $\bar{\Omega}$, of a vector field $V$ on $\bar{\Omega}$, and $a: \bar{\Omega} \rightarrow \mathbb{R}$. The first-order term $\sum_{i=1}^{n} V^{i}(x, t) \frac{\partial u}{\partial x_{i}}$ is invariant under change of coordinates. We shall assume throughout that $V(x, t)$ and $a(x, t)$ describe real-analytic mappings from $t$ to the space of smooth vector fields and smooth functions, resp., on $\bar{\Omega}$.

THEOREM 5.1.2. Theorem 5.1.1 continues to be valid if Equation (5.1.1) is replaced by Equation (5.1.4).

As in the proof of Theorem 5.1.1 (sketched in Section 5.5) will show this result de facto provides sufficient conditions implying the geometric optics condition [Lit.1], [B-L-R.1], which originally was given in $\mathbb{R}^{n}$. The proof of [L-T.1] continues to hold true in the Riemannian setting of this present section. The conditions of Theorem 5.1.1 are far from necessary, and indeed, examples are given in [G-L.1] and below where controllability holds without the condition of the uniqueness of the chords being satisfied.

5.2. Chord uniqueness and nondegeneracy. The relevant definitions are as follows:

DEFINITION 1. The chord is a length-minimizing geodesics of $\bar{\Omega}$ joining two given points of its boundary $\partial \Omega$.

The existence of a chord joining any two points of $\partial \Omega$ is well-known, although in general it may have non-geodesic segments lying in $\partial \Omega$ (see, e.g., [DoC.1, pp. 147-148]). If, however, we assume that $\partial \Omega$ has positive second fundamental form as in (i), then for any length-minimizing curve $\sigma:\left[s_{0}, s_{1}\right] \rightarrow \bar{\Omega}$ between two points of $\bar{\Omega}$, we have $\sigma\left(\left(s_{0}, s_{1}\right)\right) \subset \Omega$ (see [G-L.1, Corollary 3.3 or Lemma 4.1]). In particular, every chord of $\bar{\Omega}$ is a geodesic in this case.

As restatement of assumption (ii) above is that any two points of $\partial \Omega$ are connected by at most one (and hence exactly one) chord.

Definition 2. Two points $\gamma\left(s_{1}\right)$ and $\gamma\left(s_{2}\right)$ of a geodesic $\gamma$ are called conjugate points if $s_{1} \neq s_{2}$ and there exists a nontrivial Jacobi field $J$ along $\gamma$ with $J\left(s_{1}\right)=0$ and $J\left(s_{2}\right)=0$. [A Jacobi field is a solution to the linearized geodesic equation, or Jacobi equation.] Although a chord, since it has minimum length, may in general have its endpoints conjugate to each other, no two interior points may be conjugate, by Jacobi's theorem. The case where a chord $\gamma_{0}:\left[0, a_{0}\right] \rightarrow \bar{\Omega}$ has conjugate endpoints is therefore somewhat special, and we call such a chord degenerate.

Definition 3. We say that a chord $\sigma:\left[s_{0}, s_{1}\right] \rightarrow \bar{\Omega}$ is non-degenerate if there are no conjugate points to $\sigma\left(s_{0}\right)$ along $\sigma\left(\left(s_{0}, s_{1}\right]\right)$.

Another way of viewing these hypotheses of 'chord uniqueness' and 'non-degeneracy' is as follows. Consider any two boundary points $p$ and $q$. Suppose that, among all the light rays leaving $q$ simultaneously and 
propagating in $\Omega$ (without reflection), only one ray reaches $p$ first. This is equivalent to the uniqueness of the chords. Non-degeneracy of chords is equivalent to the statement that in the situation just described, when a ray leaving $q$ turns out to be a chord from $p$ to $q$, the boundary point $p$ depends on a diffeomorphic ray in the initial direction of the ray at $q$.

5.3. Criteria for chord uniqueness and non-degeneracy. In this subsection, we collect several useful criteria which provide sufficient conditions for the hypotheses of chord uniqueness and non-degeneracy, required by the main exact controllability result under the present approach (see Theorem 5.1.1).

The first criterion is especially appropriate in a situation where observations about $\bar{\Omega}$ can only be made from its boundary:

Proposition 5.3.1. [G-L.1] Assume that $\partial \Omega$ has a positive second fundamental form. For fixed $q \in \partial \Omega$, let $\phi: \bar{\Omega} \rightarrow[0, \infty)$ be given by $\phi(x) \equiv d_{\bar{\Omega}}^{2}(x, q)$.

(1) If, for each $q \in \partial \Omega, \nabla \phi$ is continuous along $\partial \Omega$, then any two points of $\partial \Omega$ are connected by a unique chord.

(2) If, moreover, for each $q \in \partial \Omega, \nabla \phi$ is continuously differentiable along $\partial \Omega$, then the chords are non-degenerate.

Remark 5.3.1. Somewhat surprisingly, it is not true that the continuity of $\nabla \phi$ implies disconjugacy of the chords of $\bar{\Omega}$. That is, the stronger hypothesis of part (2) of Proposition 5.3.1 is required to imply the stronger conclusion. See Example 5.5 in [G-L.1].

The second, rather different, criterion concludes that chords are unique, assuming a condition which implies their nondegeneracy (compare Proposition 5.3.3 below):

Proposition 5.3.2. [G-L.1] Consider a Riemannian manifold-withboundary $\bar{\Omega}$, whose boundary has positive second fundamental form. Suppose that for all $q \in \partial \Omega$, each geodesic starting from $q$ leaves $\bar{\Omega}$ strictly before any conjugate point along the geodesic. Then $\bar{\Omega}$ has unique chords (which are nondegenerate).

(The geodesic $\gamma$ given by $\gamma(t)=\exp _{p}(t v):[0, b] \rightarrow M$ has a conjugate point at $t_{0} \in(0, b)$ if and only if $D \exp _{p}$ is singular at $t_{0} v$. Thus, on $S^{2}$, the north pole is conjugate to the south pole along any meridian.)

The next criterion refers to a general class of examples, with properties analogous to a manifold with a bulge of moderate size.

Proposition 5.3.3. [G-L.1] Let $\bar{\Omega}$ be a smooth, compact subdomain of a Riemannian manifold $M$, whose sectional curvatures at $x \in M$ are bounded above by $f(r(x))$, where $r(x)=d\left(x, x_{0}\right)$, for some $x_{0} \in \Omega$. Assume that $\exp _{x_{0}}$, the exponential map of $M$ at $x_{0}$, is defined and injective on the closed ball $\bar{B}_{R}(0) \subset T_{x_{0}} M$, and that $r(x) \leq R$ on $\bar{\Omega}$. We assume that $f:[0, R] \rightarrow \mathbb{R}$ satisfies: (1) $f$ is monotone decreasing; (2) the solution $u_{1}$ of the $O D E u^{\prime \prime}+f u=0$ with initial conditions $u_{1}(0)=1, u_{1}^{\prime}(0)=0$ remains positive on $[0, R]$; and (3) the solution $u_{2}$ of the same ODE with 
initial conditions $u_{2}(0)=0, u_{2}^{\prime}(0)=1$ has positive first derivative on $[0, R]$. Suppose also that $\partial \Omega$ has positive second fundamental form. Then $\bar{\Omega}$ has unique chords, which are non-degenerate.

COROLlary 5.3.4. Suppose that for some radius $R$, the Riemannian manifold-with-boundary $\bar{\Omega}$ satisfies the hypotheses of Proposition 5.3.3. Then the wave equation (5.1.1) on $\bar{\Omega}$ is controllable from $\partial \Omega$ in any time $T>T_{0}=2 R$.

In Section 5.1, we have noted that the Frisbee provides an example where the Riemannian wave equation is not exactly controllable from the boundary. The following criterion may be used [G-L.1, Proposition 5.4] to claim that the Frisbee example does not have unique chords.

Proposition 5.3.5. [G-L.1] Assume that $\partial \Omega$ has positive second fundamental form. Suppose there is a subdomain $\bar{D} \subset \Omega$ such that $\partial D$ has negative second fundamental form. If the dimension $n>2$, assume further that $\bar{\Omega}$ and $\bar{D}$ are rotationally symmetric. Then $\bar{\Omega}$ does not have unique chords.

\subsection{Examples.}

Example 5.4.1: Manifold with a big bulge (negative example). We have already seen in Section 5.1 that the Riemannian wave equation is not exactly controllable on a manifold with a big bulge, even when the control acts on the entire boundary. The reason is that this manifold has closed geodesics in its interior. Examining this example from the viewpoint of the sufficient conditions listed at the beginning of Section 5 , we may claim that $\bar{\Omega}$ will not have unique chords. This is proved in [G-L.1].

Example 5.4.2: Manifold with a bulge of moderate size (a gentle hill) (positive example). Let $\bar{\Omega}$ be a spherical cap, of intrinsic radius $R<\frac{\pi}{2}$, in the unit $n$-sphere $S^{n}$. Then $\partial \Omega$ has positive second fundamental form. Also, $\bar{\Omega}$ has unique chords, which are non-degenerate and have lengths $\leq 2 R$. Thus, all three assumptions at the beginning of Section 5.1 hold true. Accordingly, Theorem 5.1.1 may be applied to show that the spherical Riemannian wave equation may be controlled from the boundary in any time $T>T_{0}=2 R$. Note that the requirement $R<\frac{\pi}{2}$ is sharp, since the normal curvatures of $\partial \Omega$ equal $\cot R$, which becomes negative for $R>\frac{\pi}{2}$.

One may give a strictly convex function as well in this example. An apparently optimal choice for the spherical cap example here considered would be $v(x)=-\cos r(x)$, where $r(x)$ is the distance from $x$ to the center $x_{0}$.

Example 5.4.3: The Frisbee (negative example). We have already seen in Section 5.1 that the Riemannian wave equation defined on a Frisbee is not exactly controllable, even when the control acts on the entire boundary. The reason is that this manifold has closed geodesics in its interior. With reference to the sufficient conditions listed at the beginning of Section 5, we may add that chords will not be unique [G-L]. There is no 
strictly convex function $v: \bar{\Omega} \rightarrow \mathbb{R}$ which has positive normal derivative on $\partial \Omega$, either.

Example 5.4.4: The salt shaker or a high mountain (convex function but non-unique chords). In this rotationally symmetric example, positive sectional curvature $\equiv 1$ is concentrated in a ball $B_{R_{0}}\left(x_{0}\right)$ near the center of $\bar{\Omega}$, while the sectional curvature $K_{1}(r)$ which affects Jacobi fields along a cross-section becomes identically zero outside that ball. This allocation of curvatures is opposite to Example 5.3.3. Moreover, we shall construct the metric so that there is a convex function $v: \bar{\Omega} \rightarrow \mathbb{R}$, although there will be a conjugate points along the cross-sections, and chords will not be unique. Control is possible in a finite time, but the optimal time of control may be much less than $\operatorname{diam}_{\bar{\Omega}}(\partial \Omega)$. The example is a truncated cone, topped off at the smaller end with a spherical cap. This resembles a design for salt shakers which are commonly found in American roadside diners, for example.

It is shown in [G-L] that chords of $\bar{\Omega}$ are not unique. Thus a sufficient condition of the present "view from the boundary" approach fails.

On the other hand $\bar{\Omega}$ does support a convex function $v$ with $\frac{\partial v}{\partial \nu}>0$ on $\partial \Omega$. For example, the function $v$ may be constructed in the rotationallysymmetric form $v=\psi(r)$, with $\psi(0)=0$ and $\frac{d \psi}{d r}=u(r)$. We may apply the results of [L-T-Y.1-2], see Section 6 below, to obtain boundary control in any time greater than $T_{1}=2 \frac{\max |\nabla v|}{c_{0}}$, where $c_{0}$ is a positive lower bound on convexity of $v$, as in the second paragraph of subsection 5.2 above. With the convex function $v$ just constructed, we find $c_{0}=\cos R_{0}$ and $\max |\nabla v|=u\left(R_{1}\right)$, so that $T_{1}=2\left(L+\tan R_{0}\right)$. Here we have written $L=R_{1}-R_{0}$ for convenience.

Remark 5.4.1. [Y.1] has also used Greene-Wu's Theorem as a starting point to arrive at sufficient conditions yielding that the function $v(x)=$ $\left[d_{g}[x, x)\right]^{2}, d_{g}$ being the $g$-distance function, be strictly convex. We recall from Section 4.2 that Greene-Wu's Theorem is as follows: If $\{M, g\}$ is a smooth, noncompact complete Riemannian manifold of everywhere positive sectional curvature, then there exists a smooth strictly convex function $v$ on $\{M, g\}$.

When $M=\mathbb{R}^{n}$, it is possible to construct a metric $g$ (hence coefficients $a_{i j}$ of a second-order elliptic operator $\mathcal{A}$, see Section 6.3 below), so that by Greene-Wu's Theorem a strictly convex function $v$ exists on all of $\left\{\mathbb{R}^{n}, g\right\}$. In this case, any sufficiently smooth bounded domain $\tilde{\Omega}$ in $\mathbb{R}^{n}$ can be taken for the corresponding PDE, see Section 6.3.

Alternatively, $\left\{\mathbb{R}^{n}, g\right\}$ with non-positive sectional curvature yields $v(x)=\left[d_{g}\left(x^{0}, x\right)\right]^{2}$ to be globally strictly convex. Again, any sufficiently bounded domain $\tilde{\Omega}$ in $\mathbb{R}^{n}$ can be taken for the corresponding PDE. Additional specific non-trivial examples of globally strictly convex functions in $\left\{\mathbb{R}^{n}, g\right\}$ can be found in [Y.1], [L-T.1-2], [T-Y.1]. 
5.5. Sketch of proof of Theorem 5.1.1: Verification of the geometric optics conditions. As the reader will verify immediately, Theorems 5.1.1 and 5.1.2 follow from Propositions 5.5.1, 5.5.2, and 5.5.3 below. For the propositions below, we let $\bar{\Omega}$ be extended to become a subset of an open $n$-dimensional Riemannian manifold $M$.

Proposition 5.5.1. Let $M$ be a Riemannian manifold. Then the bicharacteristics of equations (5.1) and (5.1t) are the graphs of $M \times \mathbb{R}$ of geodesics of $M$, with unit-speed parameter identified with time $\in \mathbb{R}$. See [Du.1], p. 209.

Proposition 5.5.2. If every bicharacteristic in $\bar{\Omega} \in(0, T)$ enters or leaves $\bar{\Omega} \times(0, T)$ across the lateral boundary $\partial \Omega \times(0, T)$, then boundary control is available in any time $\geq T$. conversely, if there is a single bicharacteristic in $\Omega \times(0, T)$ that enters $\bar{\Omega} \times[0, T]$ through the open bottom $\Omega \times\{0\}$ and leaves through $\Omega \times\{T\}$, without hitting the lateral boundary, then boundary control in time $T$ is not possible.

Proof. See for example, [Lit.1] where the proof which is given for bounded domains in $\mathbb{R}^{n}$ carries over without difficulties to manifolds (this is because it involves only interior propagation of singularity results). The assumption of real analytic coefficients is easily removed for time independent coefficients. The proof in [B.1] given for $\mathbb{R}^{n}$ holds with optimal Sobolev spaces. The proof of [Lit.1] also works if the lower-order terms (i.e., not in the principal part) are real-analytic mappings from $t$ to the space of $C^{\infty}$ vector fields or $C^{\infty}$ functions on $\bar{\Omega}$. The proof should then be supplemented by uniqueness Theorem 2 of [Lit.2]. Recent results in propagation of singularities by M. Taylor (see [Tay.2]) further indicate that the required smoothness in $x$ of the coefficients can be reduced to $C^{2, a}$.

The converse follows from the propagation of singularities (see [Ra.1]). Namely, initial data can be constructed which is not $C^{\infty}$ near a point inside $\Omega$ such that this singularity is propagated along the bicharacteristic, without being affected by boundary values, to form a singularity at time $T$ inside $\Omega$. Thus the solution cannot have terminal Cauchy conditions $u(\cdot, T) \equiv 0, \frac{\partial u}{\partial t} \equiv 0$.

Remark 5.5.1. In particular, if $\Omega$ contains a closed geodesic, then boundary control is impossible in any finite time. This was the basis for the construction of counterexamples in Section 5.1.

Remark 5.5.2. It will be observed that if the hypothesis of the first part of Proposition 5.5.2 holds for a Riemannian manifold $\bar{\Omega}$, then it also holds for any compact subdomain $\bar{\Omega} \subset \Omega$.

Remark 5.5.3. Consider any compact subdomain $\bar{\Omega}_{1} \subset \Omega$, and any solution $u$ of (5.1.4) having finite energy. It follows from Remark 5.5.2 above and from Tataru's trace theorem [Ta.5] that the trace of the conormal derivative of $u$ will be in $L^{2}\left(\partial \Omega_{1} \times(0, T)\right)$. This gives us boundary control for either Neumann or Robin controls in optimal Sobolev spaces.

For example, under the hypotheses of Proposition 5.5.2, we have boundary control for either Neumann or Robin controls in optimal Sobolev spaces for $\Omega \times(0, T)$. 
Proposition 5.5.3. Assume that the boundary $\partial \Omega$ of the compact Riemannian manifold-with-boundary $\bar{\Omega}$ has a positive second fundamental form. Suppose that any two points of $\partial \Omega$ are connected by a unique chord, which is nondegenerate. Then any interior geodesic segment $\gamma:(b-\epsilon, b+$ $\epsilon) \rightarrow \Omega$ may be extended to a geodesic $\gamma:\left[s_{0}, s_{1}\right] \rightarrow \bar{\Omega}$ which is a chord, that is, which realizes the minimum length between two distinct points $\gamma\left(s_{0}\right)$ and $\gamma\left(s_{1}\right)$ in $\partial \Omega$.

The proof uses an "open and closed" connectedness argument in the unit tangent bundle in $\bar{\Omega}$.

Part III: Single PDE equations: Carleman estimates. Exact controllability. Uniform stabilization. The control-theoretic expansion of the energy method in the Riemannian metric outlined in Section 3 begins with the present Part III. In it, we shall examine, in more details and more precisely than in Section 3, each of the single classes of evolution equations, individually, and present the relevant results. Both assumptions and techniques of proof are rooted in differential geometry. The proofs are essentially self-contained, which is a further advantage of the approach. It will be clear that we have, basically, two choices of presentation. We may:

(i) either begin with a PDE evolution equation with variable coefficients as defined on a Euclidean open bounded domain of $\mathbb{R}^{n}$, and then transform it in a corresponding version on a suitable Riemannian manifold $\left(\mathbb{R}^{n}, g\right)$ (this approach is outlined in Section 6.3);

(ii) or else, we may at the outset consider a Riemannian evolution equation on a Riemannian manifold $(M, g)$ involving the corresponding Laplace-Beltrami operator, and then recover the variable coefficient case defined on a Euclidean domain as a special case.

Which form of presentation to choose is largely a subjective question of taste. Mathematically, it makes little difference in the proof, though the second approach is technically more general. Historically, as pointed out in Section 0, it was the variable coefficient case on a Euclidean domain that motivated and generated the infusion of Riemannian methods. Papers [L-T-Y.1-3], [Y.1-2], [T-Y.1] follow this route: from the Euclidean domain to the Riemannian manifold. On the other hand, papers [L-T-Y.4], [T-Y.2] follow the reverse route, by working directly on a Riemannian manifold. Here, in our presentation, we shall opt for the second approach. However, in Section 6.3, we sketch the foundation of the first approach.

6. Second-order hyperbolic equations on a Riemannian manifold: Energy methods in the Riemannian metric yielding Carleman and control estimates with lower-order terms.

6.1. Model. Euclidean versus Riemannian domain. Throughout this section, $M$ is a compact, finite-dimensional Riemannian manifold with metric $g(\cdot, \cdot)=\langle\cdot, \cdot\rangle$ and norm $|X|=\{g(X, X)\}^{\frac{1}{2}}$, and $\Omega$ is an open, bounded set of $M$ with smooth boundary $\Gamma=\Gamma_{0} \cup \Gamma_{1}$. We let $n$ 
denote the outward unit normal field along the boundary $\Gamma$. Further, we denote by $\Delta_{g}$ the Laplace (Laplace-Beltrami) operator on the manifold $M$, and by $D$ the Levi-Civita connection on $M$.

Model. In this section, we study the following Riemannian wave equation with 'energy level terms,' on $\Omega$ :

$$
\begin{aligned}
w_{t t} & =\Delta_{g} w+F(w)+f \quad \text { in }(0, T] \times \Omega \equiv Q ; \\
f \in L_{2}(Q) & =L_{2}\left(0, T ; L_{2}(\Omega)\right) .
\end{aligned}
$$

For this equation, the 'energy level' is $H^{1}(\Omega) \times L_{2}(\Omega)$ for the pair $\left\{w, w_{t}\right\}$. We define:

$$
E(t)=\int_{\Omega}\left[|D w(t)|^{2}+w_{t}^{2}(t)\right] d \Omega
$$

Accordingly, we make the following assumption:

(A.1) the 'energy level' differential term

$$
F(w)=\langle P(t, x), D w\rangle+p_{1}(t, x) w_{t}+p_{0}(t, x) w,
$$

where $P(t, \cdot)$ is a vector field on $M$ for $t>0$ and $p_{0}$ and $p_{1}$ are functions on $Q$, satisfies the following estimate: there exists a constant $C_{T}>0$, such that

$$
|F(w)|^{2} \leq C_{T}\left\{|D w|^{2}+w_{t}^{2}+w^{2}\right\}, \quad \forall x, t \in Q \text { a.e. }
$$

where $D w=\nabla_{g} w$ (the gradient of $w$ in the Riemannian metric for the scalar function $w)$. So $D w$ is a vector field, $D w \in \mathcal{X}(M)=$ the set of all vector fields on $M$. Two vertical bars $|\cdot|$ may denote the norm in the tensor space $T_{x}$ or $L_{2}(\quad)$ [He.1]. Furthermore, we may assume throughout that the forcing term $f$ in (6.1.1) satisfies $f \in L_{2}(Q) \equiv L_{2}\left(0, T ; L_{2}(\Omega)\right)$, where $d Q=d \Omega d t$, and $d \Omega$ are the volume element of the manifold $M$ in its Riemannian metric $g$.

Remark 6.1.1. Property (6.1.3b) is fulfilled if $P \in L_{\infty}(0, T, \Lambda)$, $p_{0}, p_{1} \in L_{\infty}(Q)$. In effect, we could relax the regularity assumption on the lower-order coefficient $p_{0}$ and just require that $p_{0} \in L_{p}(Q)$ for $p=$ $\operatorname{dim} \Omega+1$, by using a Sobolev embedding theorem.

6.2. Strictly convex functions and coercive fields in the Riemannian metric. We elaborate, with some overlapping and repetition, on Section 3.1, as it applies to the specific problem (6.1.1), (6.1.2).

Main assumption: Our main assumption is hypothesis (H.1) = (3.1.1) in Section 3.1: there exists a strictly convex function $v: \bar{\Omega} \rightarrow \mathbb{R}$ of class $C^{2}$, in the Riemannian metric $g$, which we take non-negative after translation, without loss of generality: $v(x) \geq 0, \forall x \in \Omega$.

Under the common setting of Section 3.2, two related (or closely related) yet different energy methods in the Riemannian metric have become 
available. We shall treat them separately in Sections 6.4-6.5 and in Section 7, respectively, after pointing out explicitly how the variable coefficient, second-order hyperbolic equation defined on a Euclidean open bounded domain is contained in the abstract treatment of Sections 6.1 and 6.2.

6.3. A second-order hyperbolic equation with variable coefficients defined on a Euclidean domain. In this section $\tilde{\Omega}$ is an open bounded domain in $\mathbb{R}^{n}$, with boundary $\partial \tilde{\Omega}=\tilde{\Gamma}$ of class, say, $C^{2}$. Let $x=\left[x_{1}, \ldots, x_{n}\right]$, and let, as in $(2.2 .2)$ :

$$
\begin{aligned}
\mathcal{A} w & =-\sum_{i, j=1}^{n} \frac{\partial}{\partial x_{i}}\left(a_{i j}(x) \frac{\partial w}{\partial x_{j}}\right), \\
\sum_{i, j=1}^{n} a_{i j}(x) \xi_{i} \xi_{j} & \geq a \sum_{i=1}^{n} \xi_{i}^{2}, \quad x \in \tilde{\Omega},
\end{aligned}
$$

be a second-order differential operator, with real coefficients $a_{i j}=a_{j i}$ of class $C^{1}$, see Remark 6.3.1, satisfying the uniform ellipticity condition for some positive constant $a>0$. Thus, we can extend $a_{i j}(x)$ smoothly to all of $\mathbb{R}^{n}$ so that the matrices

$$
\begin{gathered}
A(x)=\left(a_{i j}(x)\right) ; \quad G(x) \equiv[A(x)]^{-1}=\left(g_{i j}(x)\right), \\
i, j=1, \ldots, n, x \in \mathbb{R}^{n}
\end{gathered}
$$

are positive definite on any $x \in \mathbb{R}^{n}$. The second-order hyperbolic equation defined on $\tilde{\Omega}$ is

$$
w_{t t}+\mathcal{A} w=F(w) \quad \text { in }(0, T] \times \tilde{\Omega},
$$

with $\mathcal{A}$ defined by (6.3.1) and $F(w)$ the first-order differential operator defined by (2.2.3).

Riemannian metric. Let $\mathbb{R}^{n}$ have the usual topology and $x=$ $\left[x_{1}, x_{2}, \ldots, x_{n}\right]$. For each $x \in \mathbb{R}^{n}$, define the inner product and the norm on the tangent space $\mathbb{R}_{x}^{n} \equiv \mathbb{R}^{n}$ via $(6.3 .2)$ by

$$
\begin{gathered}
g(X, Y)=\langle X, Y\rangle_{g} \equiv \sum_{i, j=1}^{n} g_{i j}(x) \alpha_{i} \beta_{j}, \quad \forall X, Y \in \mathbb{R}_{x}^{n} \\
|X|_{g}=\left\{\langle X, X\rangle_{g}\right\}^{\frac{1}{2}} ; \quad X=\sum_{i=1}^{n} \alpha_{i} \frac{\partial}{\partial x_{i}}, \quad Y=\sum_{i=1}^{n} \beta_{i} \frac{\partial}{\partial x_{i}} \in \mathbb{R}_{x}^{n}
\end{gathered}
$$

Then $\left(\mathbb{R}^{n}, g\right)$ is a Riemannian manifold with Riemannian metric $g$. One may prove that [Y.2], [L-T-Y.4]

$$
\mathcal{A} w=-\Delta_{g} w+D w,
$$

$\Delta_{g}=$ corresponding Laplace-Beltrami operators; that is, under the change of metric, from the original Euclidean metric to $g$, we have that the secondorder elliptic operator (6.3.1) becomes the Laplace-Beltrami operator on 
$\left(\mathbb{R}^{n}, g\right)$, modulo a first-order term. Thus Eqn. (6.3.3) is turned into (6.1.1) on $\left(\mathbb{R}^{n}, g\right)$, where $(6.1 .3 \mathrm{~b})$ is satisfied. This shows the benefit accrued by the change of metric: The energy method is conducted with respect to $\Delta_{g}$.

Remark 6.3.1. Let the coefficients $a_{i j}$ in (6.3.1) be of class $C^{1}$, as assumed. Then the entries $g_{i j}$ in (6.3.4) are of class $C^{1}$ as well. Thus, the connection coefficients (Christoffel symbols) $\Gamma_{i k}^{\ell}$, see [DoC.1] are of class $C^{0}$. The geodesic-solutions to a corresponding second-order nonlinear ordinary differential equation [DoC.1] are then of class $C^{2}$. Thus, the square of the distance function $d_{g}^{2}\left(x, x_{0}\right)$ is in $C^{2}$. Typically, but by no means always, the required strictly convex function is taken to be $d_{g}^{2}\left(x, x_{0}\right)$, under suitable assumptions on the sectional curvature. See Section 4. We also notice that in our case, where the manifolds are complete, the geodesics exist globally.

Remark 6.3.2. We may actually start with Eqn. (6.3.1) on a Riemannian manifold $\left\{M, g_{1}\right\}$. After a change similar to the one in (6.3.2), we obtain (6.1.1) on $\{M, g\}$.

6.4. Carleman estimates for problem (6.1.1), (6.1.2) with lower-order terms.

THEOREM 6.4.1. [L-T-Y.1-2] (Carleman estimate, first version) With reference to Eqn. (6.1.1), assume $(A .1)=(6.1 .3)$ on the energy level term $F(w) ;(H .1)=(3.1 .1)$ on the strictly convex function $v$; and $f \in$ $L_{2}(Q)$ as in (6.1.1). Let $w$ be a solution of (6.1.1) in the following class

$$
\left\{\begin{array}{l}
w \in H^{1,1}(Q) \equiv L_{2}\left(0, T ; H^{1}(\Omega)\right) \cap H^{1}\left(0, T ; L_{2}(\Omega)\right) ; \\
w_{t}, \frac{\partial w}{\partial n} \in L_{2}\left(0, T ; L_{2}(\Gamma)\right) .
\end{array}\right.
$$

Let $\phi(x, t)$ be the function defined by (3.2.1), so that $T>T_{0}$, with $T_{0}$ defined by (3.2.2). Let $E(t)$ be the 'energy' (6.1.2). Then, for all values $\tau>0$, the following one-parameter family of estimates holds true:

$$
B_{\Sigma}(w)+\frac{C_{T}}{\tau} \int_{Q} e^{\tau \phi} f^{2} d Q+T C_{T, \tau}\|w\|_{C\left([0, T] ; L_{2}(\Omega)\right)}^{2}
$$

$$
\begin{aligned}
& \geq\left(\rho-c-\frac{C_{T}}{\tau}\right) \int_{0}^{T} \int_{\Omega} e^{\tau \phi}\left[|D w|^{2}+w_{t}^{2}\right] d Q-C(1+\tau) e^{-\delta \tau}[E(T)+E(0)] \\
& \geq\left(\rho-c-\frac{C_{T}}{\tau}\right) e^{-\frac{\tau \delta}{2}} \int_{t_{0}}^{t_{1}} E(t) d t-C(1+\tau) e^{-\delta \tau}[E(T)+E(0)],
\end{aligned}
$$

where $0<c<\rho$ as in (3.2.2) and $\delta>0$ in (3.2.4). Moreover, the boundary terms $B_{\Sigma}(w)$ are given explicitly by

$$
\begin{aligned}
B_{\Sigma}(w)= & \int_{\Sigma} e^{\tau \phi} \frac{\partial w}{\partial n}\left[D v(w)-\phi_{t} w_{t}\right] d \Sigma+\int_{\Sigma} \frac{\partial w}{\partial n} w\left[\frac{1}{2} \mu-(1+c) e^{\tau \phi}\right] d \Sigma \\
& +\frac{1}{2} \int_{\Sigma} e^{\tau \phi}\left[w_{t}^{2}-|D w|^{2}\right]\langle D v, n\rangle d \Sigma
\end{aligned}
$$


where $\mu(x, t)$ is a suitable function depending on $\phi$, see [L-T.17], [L-T-Y.2]. (Eqn. (6.4.2a) is obtained by use also of property (3.2.4) of $\phi$. To obtain (6.4.2b) from (6.4.2a), one further uses property (3.2.5) of $\phi$.

A related result is in [Ta.7] in the pseudo-differential language. Our Theorem 6.4.1 has a more friendly proof and the estimate is more explicit.

Remark 6.4.1. (Idea of proof) [L-T-Y.1-2] The proof of Theorem 6.4.1 is by energy method in the Riemannian metric: it uses the following main multipliers, where $D v=D \phi$, recalling (3.2.1):

$$
e^{\tau \phi(x, t)}\left[\langle D v, D w\rangle-\phi_{t} w_{t}\right] ; \quad\left[\operatorname{div}\left(e^{\tau \phi} D v\right)-\frac{d}{d t}\left(e^{\tau \phi} \phi_{t}\right)\right] w .
$$

That is, one multiplies (6.1.1) by the multipliers in (6.4.4) and integrates by parts. They represent a far-reaching generalization of the energy methods (multipliers) used since the 'classical' period of early/mid-80's:

(1) Under the following two conditions, that (i) Eqn. (6.1.1) is defined on a Euclidean domain $\Omega$ (thus $\Delta_{g}=\Delta=$ the usual Laplacian, $g_{i j}=\delta_{i j}$ ), and moreover, (ii) $F(w) \equiv 0$ (no energy level terms!), then we recover the 'classical' main multipliers of the early/mid-80's: $h \cdot \nabla w, w$ div $h$, where $h(x)$ is a smooth coercive vector field on $\Omega$, not necessarily conservative (i.e., not necessarily $h=\nabla v$, for some scalar function $v(x)$ ) [L-L-T.1], [LT.1], [L-T.3], [Tr.1], [Ho.1] These are special cases of (6.4.4) when $\tau=0$ (that is, no free parameter is used) and the metric is Euclidean.

Prior to energy methods efforts in establishing Continuous Observability Inequalities (0.3), the multiplier $h \cdot \nabla w,\left.h\right|_{\Gamma}=\nu$, had been critically used in [L-L-T.1] to obtain the trace regularity inequality; this is the reverse inequality (for all $T>0$ ) of the C.O.I. (6.5.3) below [which is the present specialization of the C.O.I. (0.3)]. By duality, the reverse inequality yields the optimal regularity (6.5.8) of second-order hyperbolic equations with Dirichlet control. This work was instrumental in spurring further research in C.O.I. In fact, the proof of [L-L-T.1], leading to a key energy identity, serves also as a main first component of the proof of the C.O.I. (0.3). The second part of the proof of the C.O.I. (0.3) uses the second main multiplier $w \operatorname{div} h$ to take care of the difference: "kinetic energy - potential energy."

Control-theoretic inequalities - C.O.I. (0.3) and U.S.I. (0.5)versus reverse trace regularity inequalities. The following considerations are of paramount importance in understanding the topic at hand. The proof of the reverse trace regularity works equally well for variable coefficients (even all depending on time and space!) with the appropriate regularity requirement [L-L-T.1], as noted in (6.5.8). By stark contrast, the presence of variable coefficients in both principal part and/or energy level terms introduces most serious additional difficulties over the constant coefficient, canonical case in showing the relevant control-theoretic inequalities: for instance the C.O.I. (6.5.3) (Dirichlet case), or (6.5.6) (Neumann case); or the U.S.I. (6.5.16) (Neumann case) or (6.6.10) (Dirichlet case). These 
considerations apply to all present classes of evolution equations, not only second-order hyperbolic equations.

(2) Generalizing case (1) in one direction, we let now $\Delta_{g}=\Delta$ still but $F(w)$ be a first-order operator satisfying the Euclidean version of (6.1.3b): In this case, the exact Euclidean versions of the multipliers in (6.4.4) were used in [L-T.17], following the general approach, based on pseudo-differential calculus, of [Hor.1-2] (solutions with compact support) and [Ta.1-3] (solutions with traces).

(3) Generalizing case (1) in another direction, we return to (6.1.1) defined on a manifold this time with $F(w) \equiv 0$. Then the multiplier $\langle H, D w\rangle_{g}$, $H$ coercive vector field as in assumption $\left(\mathrm{H} .1^{\prime}\right)=(3.1 .2)$, was used in [Y.1] in the context of Section 6.3, Eqn. (6.3.3) with $F(w) \equiv 0$, a critical assumption for the method of [Y.1] to work.

(4) Finally, the combination of case (2) and case (3) leads to the multipliers in (6.4.4) for the full Eqn. (6.1.1), first used in [L-T-Y.1-2]. The proof in this case is a parallel development of that in [L-T.17]; computations in the Euclidean metric performed in [L-T.17] are replaced by counterpart computations in the Riemannian metric $g$, using the Levi-Civita connection $D$.

More historical details are given in the introduction of [L-T-Y.2].

By far, the bulk of the proof of the control-theoretic inequalities of the forthcoming Section 6.5 rests with the Carleman estimates, first version, of Theorem 6.4.1. It is particularly in achieving this result that the Riemannian geometric energy method, based on the multipliers in (6.4.4), plays a critical role. The improvement from the first to the second version of the Carleman estimate, as in Theorem 6.4.2 below, is more routine for second-order hyperbolic equations, see below.

THEOREM 6.4.2. (Carleman estimate, second version) Assume the hypotheses and the notation of Theorem 6.4.1. Then, for all $\tau>0$ sufficiently large, there exists a constant $k_{\phi, \tau}>0$ (recall $c<\rho$ from (3.2.2)) such that the following one-parameter family of estimates holds true:

$$
\begin{aligned}
\bar{B}_{\Sigma}(w) & +\frac{C_{T}}{\tau} \int_{Q} e^{\tau \phi} f^{2} d Q+C_{T, \tau}\|w\|_{L_{2}\left(0, T ; L_{2}(\Omega)\right)}^{2} \\
(6.4 .5 \mathrm{a}) & \geq e^{-\frac{\tau \delta}{2}}\left\{\left(\rho-c-\frac{C_{T}}{\tau}\right) \frac{e^{-C_{T} T}}{2}\left(t_{1}-t_{0}\right)-C(1+\tau) e^{-\frac{\tau \delta}{2}}\right\}[E(T)+E(0)] \\
(6.4 .5 \mathrm{~b}) & \geq k_{\phi, \tau}[E(T)+E(0)],
\end{aligned}
$$

where, recalling $B_{\Sigma}(w)$ from (6.4.3), the boundary terms $\bar{B}_{\Sigma}(w)$ are given by

$$
\bar{B}_{\Sigma}(w)=B_{\Sigma}(w)+\text { const }_{\phi, \tau} \int_{\Sigma}\left|\frac{\partial w}{\partial n} w_{t}\right| d \Sigma
$$


a 'brutal' estimate of which is

$$
\left|\bar{B}_{\Sigma}(w)\right| \leq C_{\phi} \int_{\Sigma}\left[|D w|^{2}+w_{t}^{2}+w^{2}\right] d \Sigma
$$

Assume, further, that $\Gamma=\overline{\Gamma_{0} \cup \Gamma_{1}} \equiv \partial \Omega, \Gamma_{0} \cap \Gamma_{1}=\emptyset$ and that the solution $w$ of Eqn. (6.1.1) satisfies

$$
\left.w\right|_{\Sigma_{0}} \equiv 0, \Sigma_{0}=(0, T] \times \Gamma_{0} \text {, and that }\langle D v, n\rangle \leq 0 \text { on } \Gamma_{0},
$$

where $n(x)$ is the unit outward normal field at $x \in \Gamma$. Then: estimate (6.4.5) holds true for all $\tau>0$ sufficiently large with the boundary terms $\bar{B}_{\Sigma}(w)$ replaced by the boundary terms $\bar{B}_{\Sigma_{1}}(w)$, that is, evaluated only on $\Sigma_{1}=(0, T] \times \Gamma_{1}, \Gamma=\Gamma_{0} \cup \Gamma_{1}$, with $\Gamma_{0}, \Gamma_{1}$ open disjoint subsets of $\Gamma$; while $\bar{B}_{\Sigma_{0}}(w) \leq 0$.

Again, a related result is in [Ta.7] in the pseudo-differential language.

Idea of proof. [L-T.17], [L-T-Y.1-2] By multiplying Eqn. (6.1.1) by $w_{t}$ we arrive at inequality (3.2.7), from which, using Gronwall inequality, we obtain the inequality

$$
\begin{aligned}
|E(t)-E(s)| & \leq \Lambda(T) e^{C_{T}(t-s)}, \quad 0 \leq s \leq t \leq T \\
\Lambda(T) & =\int_{0}^{T} \int_{\Omega} f^{2} d Q+2 \int_{0}^{T} \int_{\Gamma}\left|\frac{\partial w}{\partial n} w_{t}\right| d \Sigma+C_{T}\|w\|_{L_{2}(Q)}^{2},
\end{aligned}
$$

where $\Lambda(T)$ is the present specialization of $G(T)$ in (3.2.7). ¿From here we obtain [L-T.17], [L-T-Y.1-2]

$$
E(t) \geq \frac{E(0)+E(T)}{2} e^{-C_{T} T}-\Lambda(T), \quad 0 \leq t \leq T
$$

which substituted in the right side of $(6.4 .2 \mathrm{~b})$ yields readily $(6.4 .5)$, with $\tau$ sufficiently large.

6.5. Control-theoretic results for (6.1.1)-(6.1.3). We preliminarily let $\partial \Omega \equiv \Gamma=\overline{\Gamma_{0} \cup \Gamma_{1}}, \Gamma_{0} \cap \Gamma_{1}=\emptyset, \Gamma_{0}, \Gamma_{1}$ open in $\Gamma$, where $\Gamma_{0}$ is defined by (6.4.7), that is:

$$
\Gamma_{0}=\{x \in \Gamma:\langle D v, n\rangle \leq 0\},
$$

with $n(x)$ the unit outward normal field at $x \in \Gamma$, where $v$ is the strictly convex function of assumption $($ H.1 $)=(3.1 .1)$, in the Riemannian metric $g$.

Continuous observability inequalities.

THEOREM 6.5.1. (Dirichlet case) Assume the setting of Section 6.1: hypothesis $(H .1)=(3.1 .1)$ (thus $T>T_{0}$ with $T_{0}$ given by (3.2.2)) and $(A .1)=(6.1 .3)$ on $F$. Let $f \equiv 0$. Assume further that (6.1.1) possesses the following unique continuation property: if $\psi$ is a solution of (6.1.1) in 
the class (6.4.1) which satisfies the over-determined B.C., with $\Gamma_{0}$ as in (6.5.1):

$$
\left.\psi\right|_{\Sigma} \equiv 0 \text { and }\left.\frac{\partial \psi}{\partial n}\right|_{\Sigma_{1}} \equiv 0, \quad \Gamma_{1}=\Gamma \backslash \Gamma_{0},
$$

then, in fact, $\psi \equiv 0$ in $Q$. Finally, let $w$ be a solution of (6.1.1) in the class (6.4.1) which satisfies the homogeneous Dirichlet B.C.: $\left.w\right|_{\Sigma} \equiv 0$. Then, the following continuous observability inequality holds true for $T>T_{0}$ : there exists $C_{T}>0$ such that

$$
\int_{0}^{T} \int_{\Gamma_{1}}\left(\frac{\partial w}{\partial n}\right)^{2} d \Sigma_{1} \geq C_{T}\left\|\left\{w_{0}, w_{1}\right\}\right\|_{H_{0}^{1}(\Omega) \times L_{2}(\Omega)}^{2} .
$$

[This is the C.O.I. (0.3), as specialized to the present Dirichlet case.]

THEOREM 6.5.2. (Neumann case) Assume the setting of Section 6.1; hypothesis (H.1) $=$ (3.1.1) (thus $T>T_{0}$ with $T_{0}$ given by (3.2.2)) and $(A .1)=(6.1 .3)$ on $F$. Let $f \equiv 0$. Assume further that (6.1.1) possesses the following unique continuation property: if $\psi$ is a solution of (6.1.1) in the class (6.4.1) which satisfies the over-determined B.C., with $\Gamma_{0}$ as in (6.5.1):

$$
\left.\frac{\partial \psi}{\partial n}\right|_{\Sigma} \equiv 0 \text { and }\left.\psi\right|_{\Sigma_{1}} \equiv 0, \quad \Gamma_{1}=\Gamma \backslash \Gamma_{0},
$$

then, in fact, $\psi \equiv 0$ in $Q$. Finally, let $w$ be a solution of (6.1.1) in the class (6.4.1) which satisfies the following B.C.:

$$
\left.w\right|_{\Sigma_{0}} \equiv 0 \text { and }\left.\frac{\partial w}{\partial n}\right|_{\Sigma_{1}} \equiv 0, \quad \Gamma_{0} \neq \emptyset .
$$

Then, the following continuous observability inequality holds true: for $T>$ $T_{0}$, there exists $C_{T}>0$ such that

$$
\int_{0}^{T} \int_{\Gamma_{1}} w_{t}^{2} d \Sigma_{1} \geq C_{T}\left\|\left\{w_{0}, w_{1}\right\}\right\|_{H_{\Gamma_{0}}^{1}(\Omega) \times L_{2}(\Omega)}^{2} .
$$

[This is the C.O.I. (0.3), as specialized to the present Neumann case (6.5.5).]

Remark 6.5.1. We note the following sequence. One first proves the Carleman estimates (6.4.2b) and (6.4.5b) - hence, the C.O.I. (6.5.3) (Dirichlet) and (6.5.6) (Neumann) - for $H^{2,2}(Q)$-solutions $w$, as required by the computations involved. The subsequence passage from $H^{2,2}(Q)$ solutions to $H^{1,1}(Q)$-solutions is readily accomplished in the Dirichlet case, by virtue of a limit process which critically uses the "reverse inequality of (6.5.3)," which is available in this case [L-L-T.1] as discussed above. However, this passage from $H^{2,2}(Q)$ - to $H^{1,1}(Q)$-solutions is far more delicate 
in the Neumann case, where a comparable "reverse inequality of (6.5.6)" is false (in $\operatorname{dim} \Omega \geq 2$ ) [L-T.6]. The literature on continuous observability/exact controllability of the mid-80's/early 90 's seems to have overlooked this point, and unduly jumps from $H^{2,2}(Q)$-solutions to $H^{1,1}(Q)$-solutions in the Neumann C.O.I. (6.5.6) as well. A proper justification of this passage can be given, however, and can be found in [L-T-Z.1, Section 8]. See also [Ta.7]. The case of U.S.I. (6.5.16) below in the Neumann case is instead more regular: here a reverse inequality to (6.5.16) (even over the infinite time interval, not only on $[0, T]$ ) is again available at the outset, by the very property of dissipativity of the feedback problem (6.5.13) with $F(w) \equiv 0$. Hence, a limit process, as in the case of C.O.I. for the Dirichlet case, will justify the passage from $H^{2,2}(Q)$ - to $H^{1,1}(Q)$-solutions [Lag.3].

LEMma 6.5.3. (i) Let $w$ be a solution of Eqn. (6.1.1) in the class (6.4.1). Given $\epsilon>0, \epsilon_{0}>0$ arbitrarily small, given $T>0$, there exists a constant $C_{\epsilon, \epsilon_{0}, T}>0$ such that

$$
\begin{aligned}
& \int_{\epsilon}^{T-\epsilon} \int_{\Gamma}\left(\frac{\partial w}{\partial s}\right)^{2} d \Gamma d t \\
& \quad \leq C_{\epsilon, \epsilon_{0}, T}\left\{\int_{0}^{T} \int_{\Gamma}\left[\left(\frac{\partial w}{\partial n}\right)^{2}+w_{t}^{2}\right] d \Gamma d t+\|w\|_{L_{2}\left(0, T ; H^{\left.\frac{1}{2}+\epsilon_{0}(\Omega)\right)}\right.}^{2}\right\}
\end{aligned}
$$

where $\frac{\partial w}{\partial s}=\langle D w, s\rangle=$ tangential gradient.

(ii) Assume further that the solution $w$ satisfies hypothesis (6.4.7). Then the above estimate holds true with $\int_{\Gamma}$ replaced by $\int_{\Gamma_{1}}$.

Exact controllability. Dirichlet case. Consider the following mixed (controlled) problem:

$$
\begin{cases}y_{t t}=\Delta_{g} y+F(y) & \text { in } Q=(0, T] \times \Omega ; \\ y(0, \cdot)=y_{0}, y_{t}(0, \cdot)=y_{1} & \text { in } \Omega ; \\ \left.y\right|_{\Sigma_{0}} \equiv 0,\left.y\right|_{\Sigma_{1}} \equiv u & \text { in } \Sigma_{i}, i=0, i=1,\end{cases}
$$

with $F$ satisfying $($ H.1) $=$ (3.1.1). By [L-L-T.1] (which was given in the Euclidean setting of Section 6.3 but works also in the present setting where $\Omega$ is a bounded set of a Riemannian manifold $M$ ), we have the following regularity result for problem (6.5.7): the map

$$
y_{0}=0, y_{1}=0, u \in L_{2}\left(\Sigma_{1}\right) \rightarrow\left\{y, y_{t}\right\} \in C\left([0, T] ; L_{2}(\Omega) \times H^{-1}(\Omega)\right)
$$

is continuous.

Definition 6.5.1. Making Section 0 more precise, we call problem (6.5.7) "exactly controllable" over the state space $L_{2}(\Omega) \times H^{-1}(\Omega)$ (of regularity) in the interval $[0, T]$, with respect to the class of controls $L_{2}\left(0, T ; L_{2}\left(\Gamma_{1}\right)\right) \equiv L_{2}\left(\Sigma_{1}\right)$, in case the map $L_{T}, L_{T} u=\left\{y(T), y_{t}(T)\right\}$, see (6.5.8), is surjective (onto) $L_{2}(\Omega) \times H^{-1}(\Omega)$. 
A well-known result [T-L.1, p. 235] then gives the following equivalence: the above map $L_{T}$ is surjective $L_{2}\left(\Sigma_{1}\right)$ onto $L_{2}(\Omega) \times H^{-1}(\Omega)$ if and only if its adjoint $L_{T}^{*}$ is bounded below (abstract observability inequality):

$$
\left\|L_{T}^{*} z\right\|_{L_{2}\left(0, T ; L_{2}\left(\Gamma_{1}\right)\right)} \geq C_{T}\|z\|_{L_{2}(\Omega) \times H^{-1}(\Omega)},
$$

which is the present specialization of (0.3). One can readily prove (by PDEs methods or by operator methods [L-T.24, Section 10.5]) that, then, inequality (6.5.9) is equivalent to inequality (6.5.3) for the $w$-problem (6.1.1) satisfying the homogeneous B.C. $\left.w\right|_{\Sigma} \equiv 0$, as guaranteed by Theorem 6.5.1. Thus, by duality on Theorem 6.5.1, we obtain

Theorem 6.5.3. Assume $(H .1)=(3.1 .1)$ and $(A .1)=(6.1 .3)$ on $F$ and the unique continuation property of Theorem 6.5.1. Let $T>T_{0}$, with $T_{0}$ as in (3.2.2). Let $\Gamma_{0}$ be as in (6.5.1). Then the mixed problem (6.5.7) is exactly controllable in the sense of Definition 6.5.1.

Exact controllability. Neumann case. We next consider the following mixed (controlled) problem, consisting of Eqn. (6.5.7a-b) and

$$
\left.y\right|_{\Sigma_{0}} \equiv 0,\left.\quad \frac{\partial y}{\partial n}\right|_{\Sigma_{1}}=u \text { in } \Sigma_{1}, \Gamma_{0} \neq \emptyset .
$$

The case $\Gamma_{0}=\phi$ is considered in Section 7 .

Definition 6.5.2. Making Section 0 more precise, we call problem (6.5.7a-b), (6.5.10) "exactly controllable" over the (energy) space $H_{\Gamma_{0}}^{1}(\Omega) \times$ $L_{2}(\Omega)$ in the interval $[0, T]$, with respect to the class of controls $L_{2}(0, T$; $\left.L_{2}\left(\Gamma_{1}\right)\right) \equiv L_{2}\left(\Sigma_{1}\right)$, in case the corresponding map related to this problem

$$
\begin{aligned}
& y_{0}=0, \quad y_{1}=0, \\
u \in \mathcal{D}\left(\mathcal{L}_{T}\right) \subset L_{2}\left(\Sigma_{1}\right) \rightarrow & \mathcal{L}_{T} u=\left\{y(T, \cdot), y_{t}(T, \cdot)\right\} \in H_{\Gamma_{0}}^{1}(\Omega) \times L_{2}(\Omega)
\end{aligned}
$$

is surjective (onto).

In the present Neumann case, the map $\mathcal{L}_{T}$ is not continuous when $\operatorname{dim}$ $\Omega \geq 2$ [L-T.6], [Ta.5], unlike the Dirichlet case [L-L-T.1]. However, $\mathcal{L}_{T}$ is closed.

The well-known general result [T-L.1, p. 235] then gives the following equivalence: the map $\mathcal{L}_{T}$ in (6.5.11) is surjective if and only if its adjoint $\mathcal{L}_{T}^{*}$ is bounded below (abstract observability inequality):

$$
\left\|\mathcal{L}_{T}^{*} z\right\|_{L_{2}\left(0, T ; L_{2}\left(\Gamma_{1}\right)\right)} \geq C_{T}\|z\|_{H_{\Gamma_{0}}^{1}(\Omega) \times L_{2}(\Omega)}
$$

for $z \in \mathcal{D}\left(\mathcal{L}_{T}^{*}\right)$, which is the present specialization of $(0.3)$. One can readily prove (by PDE methods or by operator methods [L-T.3]) that then, inequality (6.5.12) is equivalent to inequality (6.5.6) for the $w$-problem (6.1.1) satisfying the homogeneous B.C. (6.5.5), as guaranteed by Theorem 6.5.2. Thus, by duality on Theorem 6.5.2, we obtain

Theorem 6.5.4. Assume $(H .1)=(3.1 .1)$ and $(A .1)=(6.1 .3)$ on $F$ and the unique continuation property of Theorem 6.5.2. Let $T>T_{0}$, 
with $T_{0}$ as in (3.2.2), and let $\Gamma_{0}$ be as in (6.5.1). Then the mixed problem (6.5.7a-b), (6.5.10) is exactly controllable in the sense of Definition 6.5.2.

Uniform stabilization: Neumann case. Consider the following closed loop problem with explicit dissipative feedback in the Neumann B.C.

$$
\begin{cases}w_{t t}=\Delta_{g} w+F(w) & \text { in }(0, T] \times \Omega \\ w(0, \cdot)=w_{0}, w_{t}(0, \cdot)=w_{1} & \text { in } \Omega \\ \left.w\right|_{\Sigma_{0}} \equiv 0,\left.\frac{\partial w}{\partial n}\right|_{\Sigma_{1}}=-w_{t} & \text { in } \Sigma_{i}, i=0,1\end{cases}
$$

THEOREM 6.5.5. [L-T-Y.2, p. 32] With reference to the closed loop problem (6.5.13), we have:

(i) when $\Gamma_{0} \neq \emptyset$, under assumption $(A .1)=(6.1 .3)$ for $F$, problem (6.5.13) generates a s.c. semigroup $\left\{w_{0}, w_{1}\right\} \in Y \rightarrow\left\{w(t), w_{t}(t)\right\} \in$ $C([0, T] ; Y), Y \equiv H_{\Gamma_{0}}^{1}(\Omega) \times L_{2}(\Omega)$;

(ii) when $\Gamma_{0}=\emptyset$, the same result, under $(A .1)=(6.1 .3)$ for $F$, holds true, with $Y \equiv H_{\Gamma_{0}}^{1}(\Omega) \times L_{2}(\Omega)$ replaced now by its proper subspace

$$
Y_{0} \equiv\left\{\left[u_{1}, u_{2}\right] \in Y: \int_{\Gamma} u_{1} d \Gamma+\int_{\Omega} u_{2} d \Omega=0\right\}
$$

topologized (via Green's theorem) by

$$
\left\|\left\{u_{1}, u_{2}\right\}\right\|_{Y_{0}}^{2} \equiv \int_{\Omega}\left[\left|D u_{1}\right|^{2}+u_{2}^{2}\right] d \Omega
$$

which is a norm on $Y_{0}$ (but only a semi-norm on $Y$ ) [L-T-Y.2].

(iii) Under the additional assumption (H.1) $=$ (3.1.1) and with $\Gamma_{0}$ defined by (6.5.1), and the unique continuation property in Theorem 6.5.2, the following inequality holds: for all $T$ sufficiently large, there exists a positive constant $k_{\phi, \tau}>0$ such that

$$
\int_{0}^{T} \int_{\Gamma_{1}}\left(\frac{\partial w}{\partial n}\right)^{2} d \Sigma_{1} \geq k_{\phi, \tau}\left\{\begin{array}{l}
E(T) \\
E(0)
\end{array}\right.
$$

[For $F(w) \equiv 0$, Eqn. (6.5.16) is the U.S.I. (0.5) as specialized to the present case].

Remark 6.5.2. When $F \equiv 0$ in (6.5.13a) — in which case we may well assume the weaker hypothesis $\left(\mathrm{H}^{\prime} \mathbf{1}^{\prime}\right)=(3.1 .2)$ instead of $(\mathrm{H} .1)=(3.1 .1)$ as explained there - the U.S.I. (6.5.16) implies (is equivalent to) uniform stabilization of problem (6.5.13): there exist constants $M \geq 1, \omega>0$ such that

$$
E(t) \leq M e^{-\omega t} E(0), t \geq 0,
$$

where $\sqrt{E(\cdot)}$ is the $Y \equiv H_{\Gamma_{0}}^{1}(\Omega) \times L_{2}(\Omega)$-norm in case (i) and the $Y_{0}$-norm in (6.5.14) in case (ii) for the solution $\left\{w, w_{t}\right\}$ of (6.5.13). 
6.6. Lower-level energy inequality: stabilization inequality with Dirichlet-boundary feedback in $L_{2}(\Sigma)$. In this section we treat an important situation - the stabilization problem of a wave equation (6.3.3) in its conservative form $F(w) \equiv 0$, with variable coefficient principal part and dissipative feedback in the Dirichlet-boundary condition (rather than Neumann-boundary condition as in Theorem 6.5.6). There is an important difference between the two cases: the Dirichlet-versus the Neumann - boundary feedback. The energy level in the latter is $H^{1}(\Omega) \times$ $L_{2}(\Omega)$, see Theorem 6.5.6. By contrast, the energy level of the former case has to be $L_{2}(\Omega) \times H^{-1}(\Omega)$ - the space of optimal regularity, with $L_{2}(\Sigma)$-forcing term in the Dirichlet B.C. as in (6.5.8) [L-L-T.1]. Thus, the Dirichlet-boundary feedback case requires a shift of topology downward: from $H^{1}(\Omega) \times L_{2}(\Omega)$ to $L_{2}(\Omega) \times H^{-1}(\Omega)$, for the final estimates. This is not a trivial problem. It can best be accomplished by a pseudodifferential change of variable that shifts upward $L_{2}(\Omega) \times H^{-1}(\Omega)$ to the level $H^{1}(\Omega) \times L_{2}(\Omega)$ already achieved and which is natural for energy level computations.

Dissipative model with Dirichlet boundary feedback in $L_{2}(\Sigma)$. In the notation of this Section 6, where $\Omega$ is a bounded set in the Riemannian manifold $(M, g)$, we consider the following (closed loop) Riemannian wave equation

(6.6.1a)
(6.6.1b) $\begin{cases}w_{t t}=\Delta_{g} w+f & \text { in } Q=(0, \infty) \times \Omega ; \\ w(0, \cdot)=w_{0}, w_{t}(0, \cdot)=w_{1} & \text { in } \Omega ; \\ \left.w\right|_{\Sigma_{0}} \equiv 0,\left.w\right|_{\Sigma_{1}}=\frac{\partial\left(\mathcal{A}_{0}^{-1} w_{t}\right)}{\partial n} & \text { in } \Sigma_{i}=(0, \infty) \times \Gamma_{i}, i=0,1,\end{cases}$

with dissipative feedback in the Dirichelt B.C., where

$$
\left\{\begin{array}{l}
\mathcal{A}_{0} w=\Delta_{g} w, \mathcal{D}\left(\mathcal{A}_{0}\right)=H^{2}(\Omega) \cap H_{0}^{1}(\Omega) \\
\mathcal{D}\left(\mathcal{A}_{0}^{\frac{1}{2}}\right)=H_{0}^{1}(\Omega),\left[\mathcal{D}\left(\mathcal{A}_{0}^{\frac{1}{2}}\right)\right]^{\prime}=H^{-1}(\Omega) \text { (equivalent norms) }
\end{array}\right.
$$

Here $\Gamma_{0}$ is defined with reference to the coercive field $H$ of assumption $\left(\right.$ H. $\left.1^{\prime}\right)=(3.1 .2)$, that is by

$$
\Gamma_{0}=\{x \in \Gamma:\langle H, n\rangle \leq 0\}
$$

in the Riemannian metric $g$, where $n$ is a normal field to $\Gamma$ [compare with (6.4.7)].

Remark 6.6.1. The counterpart of problem (6.6.1) in the Euclidean case is obtained as in Section 6.3 with $\Delta_{g}$ being replaced now by the operator (6.3.1).

Next, we introduce the lower-level energy $\mathcal{E}_{w}(t)$ of problem (6.1.1) defined by

$$
\mathcal{E}_{w}(t)=\|w(t)\|_{L_{2}(\Omega)}^{2}+\left\|\mathcal{A}_{0}^{-\frac{1}{2}} w_{t}(t)\right\|_{L_{2}(\Omega)}^{2}=\left\|\left\{w(t), w_{t}(t)\right\}\right\|_{Z}^{2}
$$




$$
Z \equiv L_{2}(\Omega) \times\left[\mathcal{D}\left(\mathcal{A}_{0}^{\frac{1}{2}}\right)\right]^{\prime} \equiv L_{2}(\Omega) \times H^{-1}(\Omega) \text { (equivalent norms) }
$$

We begin with a well-posedness result.

Theorem 6.6.1. [L-T.1], [L-T.12] (i) Problem (6.6.1) defines a s.c. contraction semigroup $e^{A_{D} t}$ on the space $Z$ defined by (6.6.5);

(ii) the energy $\mathcal{E}_{w}(t)$ satisfies the dissipative identity (a specialization of (0.6)),

$$
\mathcal{E}_{w}(t)+2 \int_{s}^{t}\left\langle\left. w\right|_{\Gamma_{1}}, \frac{\partial \mathcal{A}_{0}^{-1} w_{t}}{\partial n}\right\rangle_{L_{2}\left(\Gamma_{1}\right)} d \tau=\mathcal{E}_{w}(s)
$$

(iii) we have

$$
\left.w\right|_{\Gamma_{1}}=\frac{\partial \mathcal{A}_{0}^{-1} w_{t}}{\partial n} \in L_{2}\left(0, \infty ; L_{2}\left(\Gamma_{1}\right)\right)
$$

(iv) [L-T.12, Lemma 2.1]

$$
\left\|\frac{\partial w}{\partial n}\right\|_{H^{-1}\left(\Sigma_{1}\right)} \leq C_{T}\left\|\left.w\right|_{\Sigma_{1}}\right\|_{L_{2}\left(\Sigma_{1}\right)} .
$$

Next, we provide an observability-type estimate for the lower-level energy $\mathcal{E}_{w}(t)$ in (6.6.4), indeed, for the full Eqn. (6.1.1) with $f \equiv 0$.

TheOrem 6.6.2. [L-T.12], [L-T-Y.3], [Ta.1-3] Assume (H.1') $=$ (3.1.2). Let $w$ be a solution of Eqn. (6.1.1) [and with no boundary conditions imposed] within the class

$$
\left\{\begin{array}{l}
\left\{w, w_{t}\right\} \in C\left([0, T] ; L_{2}(\Omega) \times H^{-1}(\Omega)\right) ; \\
\left.w\right|_{\Sigma_{1}} \in L_{2}\left(\Sigma_{1}\right) ;\left.\frac{\partial w}{\partial n}\right|_{\Sigma_{1}} \in H^{-1}\left(\Sigma_{1}\right) .
\end{array}\right.
$$

Assume, moreover, condition (6.6.3).

Then the following inequality holds true: If $T$ is large enough, then

$$
\begin{aligned}
\int_{0}^{T} \mathcal{E}_{w}(t) d t \leq C_{T}\{ & \int_{0}^{T} \int_{\Gamma_{1}} w^{2} d \Sigma_{1}+\left\|\frac{\partial w}{\partial n}\right\|_{H^{-1}\left(\Sigma_{1, T}\right)}^{2} \\
& \left.+\|w\|_{H^{-1}(Q)}^{2}+\|f\|_{L_{2}\left(0,1 ; H^{-1}\left(\Sigma_{T}\right)\right.}^{2}\right\} .
\end{aligned}
$$

The notation on the norm of $f$ means: $L_{2}$ in the normal direction and $H^{-1}$ in the tangential direction and time [Hor.2].

We now return to the feedback problem (6.6.1) with $\left\{w_{0}, w_{1}\right\} \in Z$ : By Theorem 6.6.1, we see that conditions (6.6.9) are satisfied. We can then apply estimate (6.6.10) supplemented by (6.6.8), where we use (6.6.1c) on $\Sigma_{1}$. We thus obtain the U.S.I. (0.5) polluted by the lower-order term: $w \in$ $H^{-1}(Q)$. This lot is then absorbed by a standard compactness/uniqueness 
argument, where the uniqueness is provided by elliptic theory [Hor.2, p. 14] for the static problem corresponding to (6.6.1a). This way, the desired uniform stabilization problem is recovered as a special case when the dissipative B.C. (6.6.1c) on $\Sigma_{1}$ is invoked. We obtain

THEOREM 6.6.3. In reference to problem (6.6.1), assume $\left(H .1^{\prime}\right)=$ (3.1.2) as well as (6.6.3) on $\Gamma_{0}$. Then, the s.c. contraction semigroup $e^{A_{D} t}$ guaranteed by Theorem 6.6.1 is uniformly stable on the space $Z$ defined in (6.6.5): there exists constants $M \geq 1, \omega>0$ such that

$$
\left\|e^{A_{D} t}\right\|_{\mathcal{L}(Z)} \leq M e^{-\omega t} ; \text { or } \mathcal{E}_{w}(t) \leq M e^{-\omega t} \mathcal{E}_{w}(0), t \geq 0 .
$$

Remark 6.6.2. Theorem 6.6.1 and Theorem 6.6.3 were first established in [L-T.1] in the Euclidean case $\Delta_{g}=\Delta=$ Euclidean Laplacian [this assumption is not critical for Theorem 6.6.1, which holds true with essentially the same proof, based on Lumer-Phillips]: in the case of Theorem 6.6.3 in the Euclidean case, $\Delta_{g}=\Delta$, the original proof in [L-T.1] required a geometrical condition: that $\Omega \subset \mathbb{R}^{n}$ be strictly convex (or the set difference of two strictly convex sets). The required shift of topology, described in the opening of this Subsection 6.6, was accomplished in [LT.1] by using an operator-theoretic global change of variable $p=\mathcal{A}_{0}^{-1} w_{t}$ to shift the needed topology $L_{2}(\Omega) \times H^{-1}(\Omega)$ for $\left\{w, w_{t}\right\}$ into the more convenient topology $H^{1}(\Omega) \times L_{2}(\Omega)$ for $\left\{p, p_{t}\right\}$, where the energy method applies. Later, a pseudo-differential change of variable was introduced in [L-T.12] from $\left\{w, w_{t}\right\}$ to $\left\{p, p_{t}\right\}$ in the aforementioned topologies, which permitted one to eliminate the geometrical conditions of [L-T.1]. Lower-level energy inequalities such as (6.6.10) were obtained in [L-T.12], [L-T-Y.3] (in a form suitable for the feedback problem (6.6.9) of interest), [Ta.1-3], [E-L-T.1], [E-L-T.4], including the RHS forcing term $f$.

A more general (and more complicated) pseudo-differential method to obtain lower-order energy estimates (with no reference to any particular uniform stabilization problem was given in [Ta.7] for general evolution equations. See also [B-L-R-H-Z.1] for second-order hyperbolic equations.

6.7. Geometric optics and strictly convex functions. Since the work of [B-L-R.1], it has been understood in specialized PDE control circles that, even in the case of the Euclidean Laplacian, a 'minimal' controlled portion of the boundary - such as is provided by the sharp geometric optics condition [B-L-R.1] - may not be expected, in general, to be obtained by using classical multipliers with vector fields which are coercive, in particular the gradients of strictly convex functions in the Euclidean metric. Several examples may be given below.

Example \#1. [G-L.2] An example is constructed in [G-L.2] of a domain in $\mathbb{R}^{2}$ with controls on the outer of the four boundary components and $g_{i j}(x) \equiv \delta_{i j}$, therefore with the Euclidean Laplacian $\Delta_{g}=\Delta$, so that control is achieved in a finite time $T_{0}$, but there is no strictly convex function $v$ on $\Omega$ with the additional property of nonpositive normal derivative 
on the uncontrolled boundary (see Figure 2 taken from [G-L.2]). In fact, the analysis of [B-L-R.1], [Lit.1] shows that control may be achieved in a time equal to the maximum length of geodesics which reflect with equal angles at the uncontrolled boundary, before they cross the controlled boundary. In Figure 2 from [G-L.2], this will be achieved by a polygonal curve consisting of two line segments, each of which grazes the outer boundary curve, reflecting with equal angles at the upper boundary circle. On the other hand, a strictly convex function $v$ on $\Omega$ must have positive outward normal derivative somewhere on one of the three uncontrolled boundary circles. Namely, let the black dot in Figure 2 from [G-L.2] indicate the origin. The segments $l_{1}, l_{2}$ and $l_{3} \subset \Omega$ of the rays from the origin through the centers of the three circles meet the uncontrolled boundary at right angles at $x_{1}$, $x_{2}$ and $x_{3}$, resp. If the outward normal derivative of $v$ at $x_{k}$ is $\leq 0$, then since the restriction of $v$ to $l_{k}$ is strictly convex, the derivative of $v$ at the origin in the $x_{k}$ direction is strictly negative. But $x_{1}, x_{2}$ and $x_{3}$ do not lie in any half-plane of $\mathbb{R}^{2}$, so this contradicts the differentiability of $v$ at the origin.

On the other hand, the Euclidean Laplacian in $\mathbb{R}^{n}$ always admits infinitely many strictly convex functions, e.g., the quadratic functions $v(x)=$ $\left\|x-x_{0}\right\|^{2}$, where $x_{0}$ is any fixed point in $\mathbb{R}^{n}$. Thus, by Theorem 6.5 .4 , the above example in $\mathbb{R}^{2}$ with the Euclidean Laplacian is always exactly controllable in optimal Sobolev spaces over a time $T>T_{0}$, provided that we apply control on an additional portion of the boundary. Namely, we have to retain control on the entire portion of the boundary where $\nabla v \cdot n \geq 0$, $n$ being the unit outward normal. One symmetric way to do this is as follows. Call now $x_{0}$ the center of the domain (the dot in the picture). Then, apply Theorem 6.5.4 with the strictly convex function $v(x)=\left\|x-x_{0}\right\|^{2}$. This requires that we apply control not only on the exterior boundary, as done before, but also on the arcs of each circle illuminated by a light source at $x_{0}$.

Example \#2. [B-L-R.1] An interesting 2-d example (with the Euclidean Laplacian) is given in [B-L-R.1, p. 1031, Fig. 4]. It displays a disconnected 'minimal' portion of a circumference, which is sufficient for control in light of the geometric optics criterion. As there is no discussion, however, on whether or not such example could also be obtained by a strictly convex function and Theorem 6.5.4, we warn the reader - who may be induced to the opposite conclusion by the last paragraph on p. 1031 of [B-L-R.1] - that an argument similar to the one provided for our own Example \#1 shows likewise that Fig. 4 of [B-L-R.1] cannot be obtained by a strictly convex function.

Remark 6.7.1. A non-Euclidean example has been constructed in [Ga.1] in which control on the whole boundary in finite time is possible, but there is no strictly convex function whatever.

The example (the wiffle-ball) is as follows. Delete from the sphere a neighborhood of three closed arcs of the equator, each arc subtending an 


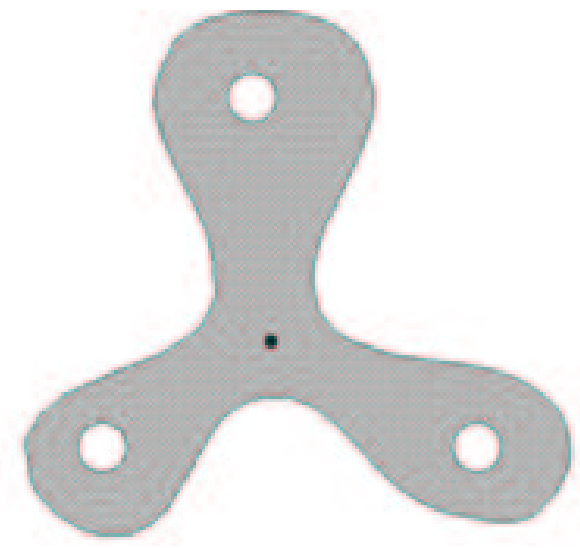

FIG. 2. The outer boundary is subject to Dirichlet controls. The three inner boundary circles are uncontrolled; instead, homogeneous Dirichlet boundary conditions are imposed. No line segment inside the domain joins distinct inner boundary circles, which implies finite-time control. But the three inner boundary circles may be reached from the point at center, in directions not lying in a half space; this implies that there is no convex function which would ensure finite-time control.

angle $\pi / 3$ from the center of the sphere and spaced an angle $\frac{\pi}{3}$ from the other two arcs. The set that remains is the domain $\Omega$. (Of course, this domain could be constructed as a planar domain, but with a non-Euclidean metric; it is easier to see the example on the sphere as a surface in $\mathbb{R}^{3}$ ). Inside $\Omega$, there are no longer any full great circles; however, there are three meridians joining the north pole to the south pole (specifically: the meridians which cross the equators at the midpoints of the three remaining arcs of the equator), meeting at each pole with equal angles $\frac{2 \pi}{3}$. The proof that there is no convex function whatever is analogous to, but more complicated than, the argument of Example \#1.

How do the above exact controllability results, Theorem 6.5.4, Theorem 6.5.5, based on the strict convexity assumption $($ H.1) $=(3.1 .1)$ [or its relaxed version $\left.\left(\mathrm{H} .1^{\prime}\right)=(3.1 .2)\right]$, compare - at least in the case of secondorder hyperbolic equations - with the exact controllability results obtained by the geometric optics approach [B-L-R.1], [Lit.1]? Since [B-L-R.1], it has been known that even in the Euclidean canonical case $\Delta_{g}=\Delta, F(w) \equiv 0$, geometric optics is more refined and general, except for requiring $C^{\infty}$ smoothness of both the Euclidean domain and the coefficients. (But see [B.1], only for the wave equation with Dirichlet control.) See Examples \#1 and \#2 and Remark 6.7.1 We point out here that paper [Ga.1] shows the following result:

THEOREM 6.7.1. [Ga.1] (i) If the boundary $\partial \Omega$ of the Euclidean domain $\Omega$ is $C^{\infty}$; (ii) if the coefficient of the second-order hyperbolic equation (6.3.3) are $C^{\infty}$ and time independent; and (iii) if one knows a-priori that 
the boundary $\partial \Omega$ does not allow infinite order of contact with the geodesics, then: the existence of a strictly convex function $v(x)$ on $\Omega$ in the Riemannian metric $g$, with $\left\langle\nabla_{g} v(x) n(x)\right\rangle_{g} \leq 0$ on $\Gamma_{0}$ as in (3.2.10) or (6.5.1) implies the remaining assumptions of $[B-L-R .1]$ and hence exact controllability on a time $T>T_{0}$, with $T_{0}$ defined by (3.2.2).

In this result, the assumption on $\partial \Omega$ having only finite order of contact with rays of geometric optics appears not to be easy to verify, in general; it holds true, however, if $\partial \Omega$ has positive definite second fundamental form. Moreover, via this route, the final exact controllability result rests with the highly technical apparatus of geometric optics and microlocal analysis in [BL-R.1] or [Lit.1]. By contrast, the proof of Theorem 3.2.2, as specialized to the exact controllability of second-order hyperbolic equations (see Section $6.5)$, is entirely self-contained.

7. Second-order hyperbolic equations: Energy method in the Riemannian metric yielding Carleman and control estimates with no lower-order terms. Purely Neumann B.C. Global unique continuation. In this section we return to the setting of (6.1.1)-(6.1.3) of a general Riemannian wave equation defined on a bounded set $\Omega$ of a Riemannian manifold $(M, g)$. We shall present a more flexible and more general method which, in contrast with the one of Section 6 , yields pointwise Carleman estimates with a controlled 'right' sign of the coefficient in front of the lower-order term, so that, eventually, the lower-order term can be dropped, yielding the advantages pointed out in Remark 3.2.2.

We shall concentrate on the most challenging case: the purely Neumann B.C. case. This is Eqn. (6.1.1) with initial data $\left\{w_{0}, w_{1}\right\} \in H^{1}(\Omega) \times$ $L_{2}(\Omega)$, and B.C.

$$
\left.\frac{\partial w}{\partial n}\right|_{\Sigma} \equiv 0 \quad \Sigma=(0, T] \times \Gamma,
$$

subject to assumption (6.1.3) for $F(w)$. The point is that we seek the C.O.I. such as (0.3) (or (6.5.6)) or the U.S.I. such as (0.5) (or (6.5.16)), involving only the subportion $\Gamma_{1}$ of $\Gamma=\overline{\Gamma_{0} \cup \Gamma_{1}}, \Gamma_{0} \cap \Gamma_{1}=\emptyset$. This corresponds to Neumann-control on $\Gamma_{1}$ and homogeneous Neumann B.C. on $\Gamma_{0}$. The easier case - typical of the literature - with homogeneous Dirichlet B.C. on $\Gamma_{0}$ is technically included as well in the present approach, indeed in a more relaxed setting: with the stringent assumption: $\langle D d, n\rangle=0$ on $\Gamma_{0}$ replaced by the more relaxed assumption: $\langle D d, n\rangle \leq 0$ on $\Gamma_{0}$. See $(\mathrm{A} .3)=(7.21)$ below. Lack of space prevents us from giving a string of the main blocks of the approach yielding the sought-after final results. Accordingly, we must refer to the original papers: [L-T-Z.1] in the case of Eqn. (6.1.1) with $\Delta_{g}=\Delta=$ the Euclidean Laplacian on $\Omega \subset \mathbb{R}^{n}$, and its generalization [T-Y.2] to the Riemannian setting as in Eqn. (6.1.1), (6.1.3). The approach in [L-T-Z.1] was inspired by [L-R-S.1, Lemma 1, p. 124], which was also used in [K-K.1]. Both works refer to the Euclidean 
setting. Even in this case, the technical "pointwise estimate" in [L-RS.1] works well for $H^{2,2}(Q)$-solutions to second-order hyperbolic equations with constant coefficient principal part and either Dirichlet control on $\Gamma_{1}$, or Neumann control on $\Gamma_{1}$, but with Dirichlet homogeneous B.C. on $\Gamma_{0}$. This is the case of the stability estimate paper [K-K.1]. The delicate passage from $H^{2,2}(Q)$ - to $H^{1,1}(Q)$-solutions is not dealt with in [K-K.1] in the critical Neumann case. (Refer to Remark 6.5.1 on this delicate passage.) By contrast, in studying the purely Neumann B.C. case-Neumann control on $\Gamma_{1}$ and Neumann homogeneous B.C. on $\Gamma_{0}$-mere application of the same pointwise estimate in [L-R-S.1] yields yes results, but under too strong geometrical conditions [I-Y.1]. The simultaneous and independent paper [L-T-Z.1] manages to considerably weaken the geometrical conditions through various additional technical points, one of which is a new and more refined version of the pointwise estimate [L-T-Z.1, Lemma 3.1]. This is too lengthy to report here. Moreover, [L-T-Z.1] performs the delicate and technical passage from $H^{2,2}(Q)$-solutions to $H^{1,1}(Q)$-solutions in the (more challenging) Neumann case. Carleman estimates of the type reported below, for use in control-theoretic results - C.O.I. and U.S.I. - with no lower-order terms were obtained in [Ta.7] in a technical paper cast in the pseudo-differential language. By contrast, all prior control theory literature contains estimates with lower-order terms (see Remark 6.5.1). Below we report results from [T-Y.2], which generalized to the Riemannian setting the treatment in the Euclidean case of [L-T-Z.1]. Only some highlights may be given here.

Assumptions. We begin with the two main assumptions yielding Carleman estimates for solutions of Eqn. (6.1.1), with no B.C.

(A.1): This is assumption $($ H.1 $)=(3.1 .1)$ which w.l.o.g. we normalize with $\rho=1$

$$
D^{2} d(X, X) \equiv\left\langle D_{X}(D d), X\right\rangle \geq 2|X|^{2}, \quad \forall x \in \Omega, \quad \forall X \in M_{x},
$$

for a strictly convex function $d: \bar{\Omega} \Rightarrow \mathbb{R}$, which in this section we take of class $C^{3}(\bar{\Omega})$.

(A.2): The assumption that $d$ has no critical point on $\bar{\Omega}$ (in the worst case, on $\left.\Gamma_{0}\right)$

$$
\inf _{x \in \Omega}|D d|=p>0
$$

in which case we shall assume w.l.o.g. the following translation/rescaling conditions

$$
\begin{gathered}
\min _{\bar{\Omega}} d(x)=m>0 ; \quad k=\inf _{\bar{\Omega}} \frac{|D d|^{2}}{d}>4, \\
\min _{x \in \bar{\Omega}, t \in\left[t_{0}, t_{1}\right]} \phi(x, t) \geq \sigma, \quad 0<\sigma<m,
\end{gathered}
$$

where we think of $m$ as being small, and we may choose $0<\sigma<m$ such that the condition on $\phi(x, t)$ in (7.4) holds true, with $\phi(x, t)$ defined 
by (3.2.1) with $v$ replaced by $d$. [This is a small technical variation over $(3.2 .5)$.]

Remark 7.1. Actually, assumption $(\mathrm{A} .2)=(7.3)$ may be dispensed with, say, in the worst case where the critical point of $d$ falls on $\Gamma_{0}$. This, however, requires a complicated procedure [L-T-Z.1, Section 10], [T-Y.2, Section 10], of which we may only give a brief sketch. We begin by writing $\Omega=\Omega_{1} \cup \Omega_{2}, \Omega_{1} \cap \Omega_{2} \neq \emptyset$, with $\Omega_{i}$ appropriately chosen. We then relax (7.3) by simply assuming the existence of two strictly convex functions $d_{i}$ : $\bar{\Omega} \rightarrow \mathbb{R}$ as in (7.2), satisfying only the weaker condition:

$$
\inf _{\Omega_{i}}\left|D d_{i}\right| \geq p>0
$$

The sets $\Omega_{i}$ need to be chosen, as to satisfy a second requirement, which is always possible to accomplish: the condition is technical, but it roughly means that each $\Omega_{i}$ is obtained by removing from $\Omega$ a small set containing the critical points of $d_{i}$ on $\Gamma_{0}$ (worst case).

Complicated cut-off functions $\chi_{i}(t, x)$ are next introduced [L-T-Z.1, Section 10], which, however, are only time-dependent (but not spacedependent) on a small internal layer of the boundary $\Gamma$. This requirement is critical in the purely Neumann B.C. case, but is not needed in the case of purely Dirichlet B.C. The results preliminarily obtained under assumptions (A.1), (A.2) are then applied to the solution $\left(\chi_{i} w\right)$ of a perturbed equation, and the estimates for $i=1,2$, are then combined.

\section{Carleman estimate, first version.}

THEOREM 7.1. [L-T-Z.1], [T-Y.2] With $0<c<1=\rho$ (chosen as in (7.2)), assume $(A .1)=(7.2)$ and $(A .2)=$ (7.3) [subject to the translation/scaling conditions (7.4)]. Let $\phi(x, t)$ be the pseudo-convex function defined by (3.2.1) with $v$ there replaced by $d$ now. Let $w \in C^{2}\left(R_{t} \times \Omega\right)$ be a solution of Eqn. (6.1.1) [and no B.C.] under the standing assumptions (6.1.3) for $F(w)$ and (6.1.1) for $f$. Then:

(i) for all $\tau>0$ sufficiently large and any $\epsilon>0$ small, the following one-parameter family of estimates holds true, with $\rho=1-c>0$ and $\beta>0$, $\beta$ depending on $\epsilon$ :

$$
\begin{gathered}
B_{\Sigma}(w)+2 \int_{0}^{T} \int_{\Omega} e^{2 \tau \phi} f^{2} d Q+C_{1, T} e^{2 \tau \sigma} \int_{0}^{T} \int_{\Omega} w^{2} d Q \\
+\left(2 \tau^{3} \beta+\mathcal{O}\left(\tau^{2}\right)-2 C_{T}\right) \int_{Q(\sigma)} e^{2 \tau \phi} w^{2} d x d t-C_{T} \tau^{3} e^{-2 \tau \delta}[E(0)+E(T)] ; \\
\quad Q(\sigma)=\{(x, t): x \in \Omega, 0 \leq t \leq T ; \phi(x, t) \geq \sigma>0\}
\end{gathered}
$$


(ii) for all $\tau>0$ sufficiently large and any $\epsilon>0$ small,

$$
\begin{aligned}
B_{\Sigma}(w) & +2 \int_{0}^{T} \int_{\Omega} e^{\tau \phi} f^{2} d Q \\
\geq & {\left[\tau \epsilon \rho-2 C_{T}\right] e^{2 \tau \sigma} \int_{t_{0}}^{t_{1}} \int_{\Omega}\left[w_{t}^{2}+|D w|^{2}\right] d \Omega d t } \\
& -C_{1, T} e^{2 \tau \sigma} \int_{0}^{T} E(t) d t-C_{T} \tau^{3} e^{-2 \tau \delta}[E(0)+E(T)] .
\end{aligned}
$$

Here, $\delta>0, \sigma>0$, and $\sigma>-\delta$ are the constants in (3.2.4) (7.7), while $C_{T}$ is a positive constant depending on $T$ and $d$. Moreover, the boundary terms $B_{\Sigma}(w), \Sigma=[0, T] \times \Gamma$, are defined by

$$
\begin{gathered}
\left.B_{\Sigma}(w)=-2 \tau \int_{\Sigma}\left(f_{1}+\theta w f_{2}\right)\langle D d, n\rangle\right] d \Sigma \\
f_{1}+\theta w f_{2}=\theta^{2}\left\{\left[|D w|^{2}-w_{t}^{2}\right]+a-\tau^{2}\left(|D d|^{2}+\phi_{t}^{2}\right)\right. \\
\left.\left.+2 \tau \ell_{t} \phi_{t}\right] w^{2}+2\left[\ell_{t}-\tau \phi_{t}\right] w w_{t}\right\} .
\end{gathered}
$$

Moreover, we have set

$$
E(t)=\int_{\Omega}\left[w_{t}^{2}+|D w|^{2}+w^{2}\right] d \Omega
$$

(iii) The above inequality may be extended to all $w \in H^{2,2}(Q)$.

The proof of the critical part (i) is given in [L-T-Z.1] in the Euclidean case and in [T-Y.2] in the Riemannian case. Here we comment explicitly on how to go from (i) to part (ii), as Eqn. (7.8) in part (ii) is the first step leading, in (7.12) of Theorem 7.2 below, to the elimination of the lower-order term.

Proof of part (ii). We take $\tau$ sufficiently large so that, since $\beta>0$, we then have that the term $\left[2 \tau^{3} \beta+\mathcal{O}\left(\tau^{2}\right)-2 C_{T}\right]$ is positive, and we then drop the corresponding lower-order interior term involving $w^{2}$ in (7.6). Moreover, we invoke the critical property $(3.2 .5)$ on $\phi$ on the first integral term on the right side of (7.6). Finally, we majorize $\int_{\Omega} w^{2}(t) d \Omega$ by $E(t)$, see (7.11), so that the term $e^{2 \tau \sigma} \int_{0}^{T} E(t) d t$ appears on the right side of (7.8).

Remark 7.1. To obtain the final version in Theorem 7.2, it is very important that the same exponent $[2 \tau \sigma]$ appears for the two exponentials outside the integrals in (7.8). This is reflected in the term $\exp (2 \tau \sigma)$ in (7.12a).

Carleman estimate, second version, without lower-order term.

ThEOREM 7.2. [L-T-Z.1], [T-Y.2] Assume the setting of Theorem 7.1, that is assumptions $(A .1)=(7.2),(A .2)=\left(\right.$ 7.3). Let $w \in H^{2,2}(Q)$ be 
a solution of Eqn. (6.1.1) [and no B.C.] under the standing assumptions (6.1.3) for $F(w)$ and (6.1.1) for $f$. Then the following one-parameter family of estimates hold true: for all $\tau$ sufficiently large, $\tau>\tau_{0}$, and any $\epsilon>0$ small, there exists a constant $k_{\phi, \tau_{0}}>0$ such that

$$
\begin{aligned}
\bar{B}_{\Sigma}(w)+ & \int_{0}^{T} \int_{\Omega} e^{2 \tau \phi} f^{2} d Q+\text { const }_{\phi} \int_{0}^{T} \int_{\Omega} f^{2} d Q \\
\geq & \left\{\left[\frac{k_{1}}{2}\left(\epsilon \tau \rho-2 C_{T}\right)\left(t_{1}-t_{2}\right) e^{-C_{T} T}\right.\right. \\
& \left.\left.\quad-\frac{C_{1, T} k_{2}}{2 k_{1}} T e^{C_{T} T}\right] e^{2 \tau \sigma}-C_{T} \tau^{3} e^{-2 \tau \delta}\right\}[E(0)+E(T)] \\
\geq & k_{\phi, \tau_{0}}[E(0)+E(T)], \quad k_{\phi, \tau_{0}}>0
\end{aligned}
$$

since $\sigma>-\delta, \delta>0$, see (7.4), (3.2.3). Here, the boundary terms $\bar{B}_{\Sigma}(w)$ are given in terms of the boundary terms $B_{\Sigma}(w)$ in (7.9):

$$
\begin{aligned}
\bar{B}_{\Sigma}(w)=B_{\Sigma}(w)+c_{T} & (\tau+1) e^{2 \tau \sigma}\left[\int_{0}^{T} \int_{\Sigma}\left|w_{t} \frac{\partial w}{\partial n}\right| d \Sigma\right. \\
& \left.+\int_{0}^{T} \int_{\Gamma_{1}}\left|w w_{t}\right| d \Sigma_{1}+\int_{t_{0}}^{t_{1}} \int_{\Gamma_{1}} w^{2} d \Gamma_{1} d t\right] .
\end{aligned}
$$

As the elimination of lot is the key goal of the present energy method approach (over the energy method approach of Section 6), we provide a sketch of the proof.

Proof. Step 1. First, we introduce

$$
\mathcal{E}(t) \equiv \int_{\Omega}\left[w_{t}^{2}+|D w|^{2}\right] d \Omega+\int_{\Gamma_{1}} w^{2} d \Gamma_{1},
$$

with $\Gamma_{1}=\Gamma \backslash \Gamma_{0}$, where $\Gamma_{0}$ is the uncontrolled, or unobserved, part of the boundary. Moreover, we note the following equivalence:

$$
k_{1} E(t) \leq \mathcal{E}(t) \leq k_{2} E(t)
$$

for some positive constants $k_{1}>0, k_{2}>0$. Next, we return to estimate (7.8) of Theorem 7.1 add the term $\left(\tau \epsilon \rho-2 C_{T}\right) e^{2 \tau \sigma} \int_{t_{0}}^{t_{1}} \int_{\Gamma_{1}} w^{2} d \Gamma_{1} d t$ to both sides, recall (7.15) for $\mathcal{E}(t)$ and obtain

$$
\begin{aligned}
B_{\Sigma}(w)+(\epsilon \tau \rho- & \left.2 C_{T}\right) e^{2 \tau \sigma} \int_{t_{0}}^{t_{1}} \int_{\Gamma_{1}} w^{2} d \Gamma_{1} d t+2 \int_{0}^{T} \int_{\Omega} e^{2 \tau \phi} f^{2} d Q \\
\geq & \left(\tau \epsilon \rho-2 C_{T}\right) e^{2 \tau \sigma} \int_{t_{0}}^{t_{1}} \mathcal{E}(t) d t \\
& \quad-C_{1, T} e^{2 \tau \sigma} \int_{0}^{T} E(t) d t-C_{T} \tau^{3} e^{-2 \tau \delta}[E(0)+E(T)] .
\end{aligned}
$$


Step 2. By multiplying (6.1.1) by $w_{t}$ and using the Gronwall inequality one obtains as in (6.4.8)-(6.4.9)

$$
\begin{aligned}
(7.17) \mathcal{E}(t) & \geq \frac{\mathcal{E}(T)+\mathcal{E}(0)}{2} e^{-C_{T} T}-N(T) \geq \frac{a}{2}[E(T)+E(0)] e^{-C_{T} T}-N(T) ; \\
(7.18) N(T) & =\int_{0}^{T} \int_{\Omega} f^{2} d Q+2 \int_{0}^{T} \int_{\Gamma}\left|\frac{\partial w}{\partial n} w_{t}\right| d \Sigma+2 \int_{0}^{T} \int_{\Gamma_{1}}\left|w w_{t}\right| d \Sigma_{1} .
\end{aligned}
$$

Similarly,

$$
E(t) \leq \frac{1}{a}\left[\frac{b(E(0)+E(T))}{2}+N(T)\right] e^{C_{T} T},
$$

and hence

$$
\begin{aligned}
-C_{1, T} e^{2 \tau \sigma} \int_{0}^{T} E(t) d t \geq & -\frac{C_{1, T} b}{2 a} T e^{C_{T} T} e^{2 \tau \sigma}[E(0)+E(T)] \\
& -\frac{C_{1, T} T}{a} e^{C_{T} T} e^{2 \tau \sigma} N(T) .
\end{aligned}
$$

Step 3. We insert (7.17) into the first integral on the right side of (7.16) and use (7.20) and readily obtain (7.13), (7.12a), by invoking (7.18) for $N(T)$.

Finally, we recall the critical relation $\sigma>0$ from (7.4), so that $\sigma>-\delta$, with $\delta>0$ defined by (3.2.3), and then $\left[\epsilon \tau e^{2 \tau \sigma}-\tau^{3} e^{-2 \tau \delta}\right]$ is positive for all $\tau$ large enough. Thus (7.12a) yields (7.12b).

So far, solutions of (6.1.1) were not required to satisfy B.C. We now introduce an additional assumption on the uncontrolled (unobserved) part $\Gamma_{0}$ of the boundary $\Gamma$. We assume

(A.3):

$$
\left.\frac{\partial d}{\partial n}\right|_{\Gamma_{0}}=\langle D d, n\rangle=0 \text { on } \Gamma \text { (Neumann case); }
$$

Assumption (A.3) $=(7.21)$ in the Neumann case (Euclidean setting) was introduced in [Tr.1, Section 5].

Remark 7.2. The question arises: which triples $\left\{\Omega, \Gamma_{0}, \Gamma_{1}\right\}$ satisfy $($ A.1 $)=(7.2),($ A.2) $=(7.3)[$ or (7.5) $]$ and $($ A.3 $)=(7.21)$ in the Neumann case? This issue is amply analyzed, in the Euclidean setting, in [L-T-Z.1, Appendices], with several classes of triples $\left\{\Omega, \Gamma_{0}, \Gamma_{1}\right\}$ given. A sufficient (but not necessary) set of conditions for a construction of a suitable $d(x)$, given in the Euclidean setting in [L-T-Z.1, Theorem A.1], admits a generalization to the Riemannian setting and is reported at the end.

Global uniqueness and continuous observability/(stabiliz -ation) inequalities in one shot. The purely Neumann case. Theorem 7.2 permits at once to get global uniqueness results for over-determined 
boundary conditions associated with Eqn. (6.1.1) with $f \equiv 0$. We consider the following over-determined problem with $\Gamma_{0}$ defined by (7.21) and $\Gamma_{1}=\Gamma \backslash \Gamma_{0}$ :

$$
\left\{\begin{aligned}
w_{t t}-\Delta_{g} w & =F(w) & & \text { in }(0, T] \times \equiv Q \\
\left.\frac{\partial w}{\partial n}\right|_{\Sigma} & \equiv 0 & & \text { in }(0, T] \times \Gamma \equiv \Sigma ; \\
\left.w\right|_{\Sigma_{1}} & \equiv 0 & & \text { in }(0, T] \times \Gamma_{1} \equiv \Sigma_{1}
\end{aligned}\right.
$$

with $F$ satisfying (6.1.3). Then, $\bar{B}_{\Sigma}(w)=0$ by $(7.22 \mathrm{~b}-\mathrm{c})$ and $(7.21)$. We obtain

Theorem 7.3. [L-T-Z.1], [T-Y.2] Assume hypotheses $(A .1)=(7.2)$, $(A .2)=(7.3),(A .3)=(7.21)$, with $\Gamma_{0}$ defined there in the Neumann case. Let $\Gamma_{1}=\Gamma \backslash \Gamma_{0}$. Let $T>T_{0}$, with $T_{0}$ defined in (3.2.2). Let $w \in H^{1,1}(Q)$ be a solution of problem (7.21). Then, in fact, $w \equiv 0$ in $Q$; indeed, in $R_{t} \times \Omega$.

The passage from $H^{2,2}(Q)$ - to $H^{1,1}(Q)$-solutions requires a non-trivial argument [L-T-Z.1, Section 8].

The purely Dirichlet case. Consider problem (7.21a) coupled with the B.C.

$$
\left.w\right|_{\Sigma} \equiv 0 ;\left.\quad \frac{\partial w}{\partial n}\right|_{\Sigma_{1}} \equiv 0
$$

where $\Gamma_{0}$ and $\Gamma_{1}$ are defined below.

TheOREM 7.4. Assume hypotheses $(A .1)=(7.2),(A .2)=(7.3)$, $(A .3)=(7.21)$ (Dirichlet), (6.1.3) for $F(w)$, where now $\Gamma_{0}$ is defined by: $\langle D d, n\rangle \leq 0$ on $\Gamma_{0}$, as in (7.21) or (3.2.10). Let $\Gamma_{1}=\Gamma \backslash \Gamma_{0}$. Let $w \in$ $H^{1,1}(Q)$ be a solution of problem (7.22a), (7.23). Then, in fact, $w \equiv 0$ in $Q$; indeed, in $\mathbb{R}_{t} \times \Omega$.

Control-theoretic inequalities.

Theorem 7.5. [L-T-Z.1], [T-Y.2] Consider Eqn. (6.1.1) with F satisfying (6.1.3). Assume hypotheses (A.1) $=$ (7.2), (A.2) $=$ (7.3), and $($ A.3 $)=(7.21)$ (Neumann). Let $\Gamma_{0}$ be defined by (7.21) (Neumann), and let $\Gamma_{1}=\Gamma \backslash \Gamma_{0}$. Let $T>T_{0}$, with $T_{0}$ defined by (3.2.2). Then the following continuous observability inequality holds true for $H^{1,1}(Q)$ solutions: there exists a constant $C_{T}>0$ such that

$$
\int_{0}^{T} \int_{\Gamma_{1}}\left[w_{t}^{2}+w^{2}\right] d \Sigma_{1}+\int_{0}^{T} \int_{\Omega} f^{2} d Q \geq c_{T} E(0),
$$

with $E(\cdot)$ defined by (7.11).

The proof of Theorem 7.5 combines Theorem 7.2, Lemma 6.5.3, and Theorem 7.3, the latter providing the uniqueness result needed in a standard compactness/uniqueness argument to absorb the interior lot $(w)$ occurring in Lemma 6.5.3. The constant $c_{T}$ in (7.24) is not explicit, a price to pay 
to avoid geometrical conditions of the literature on the observed/controlled portion of the boundary $\Gamma_{1}$.

Assumptions (A.1), (A.2), (A.3) (Neumann). In the case of Euclidean domains, several rather general classes of triples $\left\{\Omega, \Gamma_{0}, \Gamma_{1}\right\}$ are given in [L-T-Z.1, Appendices] which satisfy assumptions (A.1), (A.2), (A.3) (Neumann case).

Here we report one such class, in fact in its generalization to a Riemannian manifold, as given in [T-Y.2, Appendix B]. In the Euclidean case, the perturbation formula (7.31) was used by Tataru in the context of pseudoconvex functions by pseudo-differential methods. The Euclidean proof in [L-T-Z.1, Theorem A.4.1] is direct. It admits a Riemannian version [T-Y.2].

Setting. Let $\{M, g\}$ be an $n$-dimensional Riemannian manifold, with Levi-Civita connection $D$. Let $\Omega \subset M$ be an open, connected, compact subset of $M$, with boundary $\partial \Omega=\overline{\Gamma_{0} \cup \Gamma_{1}}, \Gamma_{0} \cap \Gamma_{1}=\emptyset$. The portion $\Gamma_{0}$ of $\partial \Omega$ is defined as follows. Let $\ell: M \rightarrow \mathbb{R}$ be a function of class $C^{2}$. Then, we define

$$
\left\{\begin{array}{l}
\Gamma_{0}=\{x \in \partial \Omega: \ell(x)=0\}, \\
\text { with the further provision that } D \ell\left(=\nabla_{g} \ell\right) \neq 0 \text { on } \Gamma_{0} .
\end{array}\right.
$$

THEOREM 7.6. In the above setting, assume that (i)

$$
D^{2} \ell(X, X)(x) \geq 0, \quad \forall x \in \Gamma_{0}, \forall X \in M_{x}
$$

(convexity of $\ell$ near $\Gamma_{0}$ );

(ii) There exists a function $d_{0}: \bar{\Omega} \rightarrow \mathbb{R}$ of class $C^{2}$, such that

(ii $\left.i_{1}\right)$

$$
D^{2} d_{0}(X, X)(x) \geq \rho_{0}|X|_{g}^{2}, \quad \forall x \in \Gamma_{0}, \forall X \in M_{x},
$$

for some constant $\rho_{0}>0$ (strict convexity of $d_{0}$ near $\Gamma_{0}$ );

(ii $)$

$$
\left.\frac{\partial d_{0}}{\partial n}\right|_{\Gamma_{0}}=\left\langle D d_{0}, n\right\rangle_{g} \leq 0 \quad \text { on } \Gamma_{0},
$$

where $n$ is the unit outward normal field to $\Gamma_{0}$ on $M$ which points in the same direction as $\nabla_{g} \ell=D \ell$. Then: there exists a function $d: \bar{\Omega} \rightarrow \mathbb{R}$ of class $C^{2}$ [which is explicitly constructed in a layer (collar) of $\Gamma_{0}$, the critical set], such that it satisfies the following two conditions:

(a)

$$
\left.\frac{\partial d}{\partial n}\right|_{\Gamma_{0}}=\langle D d, n\rangle_{g}=0 \quad \text { on } \Gamma_{0}
$$


(b)

$$
D^{2} d(X, X)(x) \geq\left(\rho_{0}-\epsilon\right)|X|_{g}^{2}, \quad \forall x \in \Gamma_{0}, \forall X \in M_{x},
$$

where $\epsilon>0$ is arbitrarily small.

Definition of $d$. The function $d(x)$ is explicitly constructed near $\Gamma_{0}$, within $\Omega$, as a perturbation of the original function $d_{0}$ assumed in (ii) above, as follows:

$$
\left\{\begin{aligned}
d(x) & \equiv d_{0}(x)+z(x) & & x \text { near } \Gamma_{0} \text { in } \Omega \\
z(x) & \equiv-\frac{\partial d_{0}}{\partial n}(\ell k)+\lambda \ell^{2}, & & k \equiv \frac{1}{|D \ell|_{g}},
\end{aligned}\right.
$$

where $\lambda$ is a sufficiently large parameter, to be selected below in the proof, while

$$
\begin{aligned}
& \frac{\partial d_{0}}{\partial n} \text { denotes an extension of }\left.\frac{\partial d_{0}}{\partial n}\right|_{\Gamma_{0}} \text { from the set } \Gamma_{0} \\
& (\text { defined by } \ell(x)=0) \text { to a layer (collar) of } \Gamma_{0} \text {, within } \Omega \text {, }
\end{aligned}
$$

which is defined by

$$
\frac{\partial d_{0}}{\partial n} \equiv\left\langle D d_{0}, n\right\rangle_{g}=\left\langle D d_{0}, \frac{D \ell}{|D \ell|_{g}}\right\rangle_{g}=\left\langle D d_{0}, k D \ell\right\rangle_{g} .
$$

$n \equiv \frac{D \ell}{|D \ell|_{g}}$ (consistently with the statement below (7.28).

8. Schrödinger equations on a Riemannian manifold: Energy methods in the Riemannian metric yielding Carleman/control estimates with lower-order terms.

Models. Assumptions. We use the same notation $(M, g),\langle\cdot, \cdot\rangle$, | $\mid, \Omega, \Gamma=\Gamma_{0} \cup \Gamma_{1}, \Delta_{g}, D$ as in Sections 6 and 7 . The philosophical strategy is the same as that of Section 6 for second-order hyperbolic equations. Thus, our pace of exposition will be brisker. However, the technicalities involved are different. In this section we study the following Schrödinger equation on a bounded set $\Omega$ of the Riemannian manifold $M$ :

$$
i w_{t}+\Delta_{g} w=F(w)+f \quad \text { in }(0, T] \times \Omega \equiv Q ; f \in L_{2}(Q),
$$

where $\Delta_{g}$ is the Laplace-Beltrami operator on $M$. For this equation, the 'energy level' is $H^{1}(\Omega)$ for $w$. We define

$$
E(t)=\int_{\Omega}|D w(t)|^{2} d \Omega
$$

Accordingly, we make the following assumption on the energy level differential term: 
(A.1) $F(w)=\langle P(t, \cdot), D w\rangle+p_{0}(t, x) w$ satisfies the following estimate

$$
|F(w)|^{2} \leq c_{T}\left[|D w|^{2}+w^{2}\right], \quad \forall t, x \in Q \text { a.e. }
$$

A remark on the coefficients such as Remark 6.1.1 applies. Furthermore, we assume $f \in L_{2}(Q)$.

We note at the outset that the 'energy level' term $F(w)$ in (A.1) is an unbounded term for Eqn. (8.1), as seen in $H^{1}(\Omega)$; while the 'energy level' term $F(w)$ in (6.1.3) for the second-order hyperbolic equation (6.1.1), as viewed in $H^{1}(\Omega) \times L_{2}(\Omega)$ is, by contrast, a bounded term. Thus, $F(w)$ is expected to give rise to more troubles in well-posedness issues in the present Schrödinger equation case. This is reflected by the need of assumption (A.2) $=(8.9)$ below.

Strictly convex function $\boldsymbol{v}(\boldsymbol{x})$. As in Sections 6.2 and 7, we assume hypothesis $(\mathrm{H} .1)=(3.1 .1)$ of Section 3.1: there exists a strictly convex (non-negative) $C^{2}$-function $v: \bar{\Omega} \Rightarrow \mathbb{R}$, so that (3.1.1) holds true.

We then define the same pseudo-convex function $\phi(x, t)$ as in (3.2.1), except that now $T>0$ is arbitrary and $c=c_{T}$ is $\operatorname{such}_{\text {that }} 4 \sup _{\Omega} v(x)<$ $c T^{2}$. Properties (i) and (ii) in (3.2.4) and (3.2.5) continue to hold true with these choices. As in Section 6.3, we see that model (8.1) includes the case of a Schrödinger equation with variable coefficients defined on an open bounded domain $\tilde{\Omega}$ of $\mathbb{R}^{n}: i w_{t}+\mathcal{A} w=F(w)+f$, on $(0, T] \times \tilde{\Omega}$, where $\mathcal{A}$ is the second-order elliptic differential operator defined by (6.3.1). We repeat the pattern of Section 6 .

\section{Carleman estimates with lower-order terms.}

THEOREM 8.1. [Tr.2], [Tr-Y.1] (Carleman estimate, first version) With reference to Eqn. (8.1), assume $(A .1)=(8.3)$ on the energy level term $F(w) ;(H .1)=(3.2 .1)$ on the strictly convex function $v(x)$; and $f \in L_{2}(Q)$ as in (8.1). Let $w$ be a solution of (8.1) in the following class

$$
\left\{\begin{array}{l}
w \in C\left([0, T] ; H^{1}(\Omega)\right), \\
w_{t} \in L_{2}\left(0, T ; H^{-1}(\Gamma)\right) ; \nabla_{\tan } w, \frac{\partial w}{\partial n} \in L_{2}\left(0, T ; L_{2}(\Gamma)\right) .
\end{array}\right.
$$

Let $\phi(x, t)$ be the function defined in (3.2.1), this time with $T>0$ arbitrary and $c=c_{T}$ sufficiently large as to have $4 \sup _{\Omega} v(x)<c T^{2}$. Let $E(t)$ be defined by (8.2). Then, for all values $\tau>0$, the following oneparameter family of estimates holds true:

$$
\begin{aligned}
B_{\Sigma}(w) & +\frac{2}{\tau} \int_{Q} e^{\tau \phi}|f|^{2} d Q+C_{T, \tau}\|w\|_{C\left([0, T] ; L_{2}(\Omega)\right)}^{2} \\
& \geq\left(\rho-\frac{c_{T}}{\tau}\right) \int_{Q} e^{\tau \phi}|D w|^{2} d Q-\frac{e^{-\delta \tau}}{\tau}[E(T)+E(0)] \\
& \geq\left(\rho-\frac{c_{T}}{\tau}\right) e^{-\frac{\tau \delta}{2}} \int_{t_{0}}^{t_{1}} E(t) d t-\frac{e^{-\delta \tau}}{\tau}[E(T)+E(0)]
\end{aligned}
$$


where the boundary terms $B_{\Sigma}(w)$ over $\Sigma=(0, T] \times \Gamma$ are given by

$$
\begin{aligned}
B_{\Sigma}(w)= & R e\left(\int_{\Sigma} e^{\tau \phi} \frac{\partial w}{\partial n} D v(\bar{w}) d \Sigma\right)-\frac{1}{2} \int_{\Sigma} e^{\tau \phi}|D w|^{2}\langle D v, n\rangle d \Sigma \\
& +\frac{1}{2}\left|\int_{\Sigma} \frac{\partial w}{\partial n} \bar{w} \operatorname{div}\left(e^{\tau \phi} h\right) d \Sigma-i \int_{\Sigma} \bar{w} w_{t} e^{\tau \phi}\langle D v, n\rangle d \Sigma\right|
\end{aligned}
$$

[Eqn. (8.5) is obtained also by use of property (3.2.4) of $\phi$. To obtain (8.6) from (8.5), one further uses property (3.2.5) of $\phi$.]

A related result is in [Ta.7], in the pseudo-differential language. Our Theorem 8.1 has a more friendly proof and the estimate is more explicit.

Remark 8.1. (Idea of proof) [T-Y.1] The proof of Theorem 8.1 is by energy method in the Riemannian metric: it uses the following main multipliers, where $D v=D \phi$, recalling (3.2.1):

$$
e^{\tau \phi(x, t)}\langle D v, D \bar{w}\rangle \text { and } \bar{w} \operatorname{div}\left(e^{\tau \phi} D v\right) \text {. }
$$

These multipliers were inspired by the second-order hyperbolic equation case, for which we refer to the multipliers (6.4.4) and Remark 6.4.1. The multipliers (8.8) represent a far-reaching generalization of the energy methods (multipliers) used for the pure Euclidean Schrödinger equation in the late '80s. The counterpart of Remark 6.4.1 applies now. More precisely:

(1) Under the following two conditions, that (i) Eqn. (8.1) is defined on a Euclidean domain $\Omega$ (thus, $\Delta_{g}=\Delta=$ the usual Laplacian, $g_{i j}=\delta_{i j}$ ) and, moreover, (ii) $F(w) \equiv 0$ (no energy level terms), then we recover the 'classical' multipliers $h \cdot \nabla \bar{w}$ and $\bar{w}$ div $h$ of [L-T.8] (Dirichlet case) with $h(x)$ a smooth coercive field on $\Omega$, by taking in (8.8) the Euclidean topology and setting $\tau=0$ (that is, no free parameter). See also [M.1] (Neumann case) with radial field.

(2) Generalizing case (1), if in (8.1) we still have $\Delta_{g}=\Delta$, but $F(w)$ is now a first-order operator satisfying the Euclidean version of (8.3), then we recover the multipliers used in [Tr.2], by taking the Euclidean metric in $(8.8)$.

It was the case in [Tr.2] that prompted the multipliers (8.8) for the general case of (8.1), (8.3), as it was previously done for second-order hyperbolic equations (Remark 6.4.1). The proof in [T-Y.2] for the general case is a parallel development of that in [Tr.2]: computations in the Euclidean metric performed in [Tr.2] are replaced by counterpart computations in the Riemannian metric $g$, using the Levi-Civita connection $D$.

A perfect counterpart of the considerations made in Remark 6.4.1 apply now regarding the control-theoretic inequalities - the C.O.I. (0.3) and the U.S.I. (0.5) — versus the reverse trace regularity inequality. If we restrict to the Dirichlet-control case, the trace regularity inequality is the reverse (for all $T>0$ ) of the C.O.I. (8.14) below, see Remark 8.2. The reverse inequality is proved in [L-T.8]; [L-T.24, Section 10.9]. The proof 
applies equally well for the case of variable coefficients (even in time and space) of appropriate regularity (as in the case of second-order hyperbolic equations). By stark contrast, variable coefficients in the principal part and/or the energy level terms introduce most serious additional difficulties over the constant coefficient, canonical case in showing the relevant control-theoretic inequalities such as (8.14) and (8.17).

As in the case of second-order hyperbolic equations of Section 6 , the bulk of the proof of the control-theoretic inequalities (8.14), (8.17) rests with the Carleman estimate, first version, of Theorem 8.1. It is particularly in achieving this result that the Riemannian geometric energy method, based on the multipliers in (8.8), plays a critical role. The improvement from the first to the second version of the Carleman estimate, as in Theorem 8.2 below, is definitely more computationally extensive and delicate than in the case of second-order hyperbolic equations (Section 6 ), since $F(w)$ is now an unbounded term for the Schrödinger Eqn. (8.1) in $H^{1}(\Omega)$. Moreover, more importantly, it requires an additional structural assumption on the energy level term $F(w)$, unlike the case of second-order hyperbolic equations. More specifically, in the present case of the Schrödinger equation (8.1), we need to impose the following structural assumption on the energy level term $F(w)$, as already pointed out in connection with estimate (3.2.7): namely that the first-order coefficient has real part that is the gradient of a scalar function (i.e., it is a conservative vector field). That is, we further assume that:

(A.2) the first-order term $F(w)$ in (8.3) is of the form

$$
F(w)=R(w)+p_{0} w, R(w)=\langle P(t, x), D w\rangle,
$$

where $\operatorname{Re} P(t, x)=\nabla \chi(t, x)$ on $\Omega$ for $t>0$ for some $W^{1, \infty}$ function $\chi$, while $P$ has imaginary part, say, time independent with $\operatorname{div}(\operatorname{Im} P) \in L_{\infty}(\Omega)$ [while $p_{0}: \Omega \rightarrow \mathbb{C}$ is a function which is in $L_{\infty}(Q)$ [or even in $L_{p}(Q)$, $p=\operatorname{dim} \Omega+1$, by using a Sobolev embedding theorem], see (8.3)]. By a change of variable on the solution, we may achieve $\nabla \chi \equiv 0$ [L-T-Z.2].

Assumption $($ A.2 $)=(8.10)$ is invoked to obtain the well-posedness relationship (3.2.7). With this inequality at one's disposal, one then obtains the counterparts of inequalities (6.4.9a) and (6.4.10). Thus, one then obtains the following result from Theorem 8.1. The results below were obtained in [Tr.2] in the Euclidean case $\Delta_{g}=\Delta\left(g_{i j}=\delta_{i j}\right)$ while the generalization to the Riemannian setting is in [T-Y.2].

THEOREM 8.2. [Tr.2], [Tr-Y.2] (Carleman estimate, second version) (a) Assume the hypotheses and setting of Theorem 8.1. In addition, assume (A.2) $=(8.9)$ and $f \in L_{2}\left(0, T ; H^{1}(\Omega)\right)$. Then, for all $\tau>0$ sufficiently large, $\tau \geq \tau_{0}>0$, there exists a constant $k_{\phi, \tau}>0$ such that the following one-parameter family of estimates holds true:

$$
\bar{B}_{\Sigma}(w)+\frac{2}{\tau} \int_{Q} e^{\tau \phi}|f|^{2} d Q+C\|f\|_{L_{2}\left(0, T ; H^{1}(\Omega)\right)}^{2}+C_{T, \phi, \tau}\|w\|_{C\left([0, T] ; L_{2}(\Omega)\right)}^{2}
$$




$$
\begin{aligned}
& \geq e^{-\frac{\tau \delta}{2}}\left\{\left(\rho-\frac{c_{T}}{\tau}\right) \frac{e^{-k T}}{2}\left(t_{1}-t_{0}\right)-\frac{e^{-\frac{\delta \tau}{2}}}{\tau}\right\}[E(T)+E(0)] \\
& \geq k_{\phi, \tau_{0}}[E(T)+E(0)],
\end{aligned}
$$

where the boundary terms $\bar{B}_{\Sigma}(w)$ are given by

$$
\bar{B}_{\Sigma}(w)=B_{\Sigma}(w)+\text { const }_{\phi, \tau, \rho} \int_{\Sigma}\left|\frac{\partial w}{\partial n}\right|\left[|W(w)|+\left|w_{t}\right|+\left|p_{0} w\right|\right] d \Sigma
$$

where $W(w)=\langle W, D w\rangle$, and where $W(x)$ is a vector field on the submanifold $\Gamma$ such that $W(x) \in \Gamma_{x}$ (the tangent space to $\Gamma$ at $x$ ) for $x \in \Gamma$.

(b) Assume further that the solution $w$ of (8.1) satisfies: $\left.w\right|_{\Sigma_{0}} \equiv 0$, $\Sigma_{0}=(0, T] \times \Gamma_{0}$, with $\Gamma_{0}$ defined by (6.5.1). Then, estimate (8.11) holds true for all $\tau>0$ sufficiently large and with the boundary terms evaluated only on $\Sigma_{1}=(0, T] \times \Gamma_{1}, \Gamma_{1}=\Gamma \backslash \Gamma_{0}$, while the boundary terms evaluated on $\Sigma_{0}$ are negative: $\bar{B}_{\Sigma_{0}}(w) \leq 0$.

Control-theoretic results. We preliminarily let $\partial \Omega \equiv \Gamma=\overline{\Gamma_{0} \cup \Gamma_{1}}$, $\Gamma_{0} \cap \Gamma_{1}=\emptyset, \Gamma_{0}, \Gamma_{1}$ open in $\Gamma$, where $\Gamma_{0}$ is defined, as in (6.5.1) or (6.4.7), by $\Gamma_{0}=\{x \in \Gamma:\langle D v, n\rangle \leq 0\}$, with $n(x)$ the unit outward normal field at $x \in \Gamma$, where $v$ is the strictly convex function of assumption (H.1) $=$ (3.1.1), in the Riemannian metric $g$.

\section{Continuous observability inequalities.}

THEOREM 8.3. (Dirichlet case) Assume the above setting: hypothesis (H.1) - (3.1.1), $(A .1)=(8.3)$, and $(A .2)=(8.9)$. Let $T>0$, and let $\Gamma_{0}$ be defined as above (in (6.5.1)). Let $f=0$. Assume further that (8.1) possesses the following unique continuation property: if $\psi$ is a solution of (8.1) in the class (8.4), which satisfies the over-determined B.C. (as in (6.5.2)):

$$
\left.\psi\right|_{\Sigma} \equiv 0 \text { and }\left.\frac{\partial \psi}{\partial n}\right|_{\Sigma_{1}} \equiv 0 \text { on } \Gamma_{1}=\Gamma \backslash \Gamma_{0},
$$

then, in fact, $\psi \equiv 0$ in $Q$. Finally, let $w$ be a solution of (8.1) in the class (8.4) which satisfies the homogeneous Dirichelt B.C.: $\left.w\right|_{\Sigma} \equiv 0$. Then, the following continuous observability inequality holds true: there exists $C_{T}>0$ such that

$$
\int_{0}^{T} \int_{\Gamma_{1}}\left(\frac{\partial w}{\partial n}\right)^{2} d \Sigma_{1} \geq C_{T}\left\|w_{0}\right\|_{H_{0}^{1}(\Omega)}^{2} .
$$

[This is the C.O.I. (0.3), as specialized to the present Dirichlet case.]

Remark 8.2. The converse (trace regularity) of inequality (8.14) always holds true, for any $T>0$ for (8.1), (8.3) with $\left.w\right|_{\Sigma} \equiv 0$ [L-T.8], [L-T.24, Section 10.9].

Remark 8.3. In case of the pure Schrödinger equation, $F(w) \equiv 0$, in the Euclidean setting, the C.O.I. (8.14) is shown in [Le.2] under a geometric optics condition. 
TheOREM 8.4. (Neumann case) We assume the same hypotheses of Theorem 8.3, thus $($ H.1) $=(3.1 .1),($ A.1 $)=(8.3),($ A.2) $=(8.9), T>0$, $\Gamma_{0}$ as in (6.5.1)], except that the present version of the unique continuation property is as follows: let $\psi$ be a solution of (8.1) in the class (8.4) satisfying the over-determined B.C. (same as (6.5.4)):

$$
\left.\frac{\partial \psi}{\partial n}\right|_{\Sigma} \equiv 0 \text { and }\left.\psi\right|_{\Sigma_{1}} \equiv 0, \Gamma_{1}=\Gamma \backslash \Gamma_{0},
$$

then, in fact, $\psi \equiv 0$ in Q. Finally, let $w$ be a solution of (8.1) in the class (8.4) which satisfies the homogeneous Neumann

$$
\left.w\right|_{\Sigma_{0}}=0 ;\left.\quad \frac{\partial w}{\partial n}\right|_{\Sigma_{1}} \equiv 0, \quad \Gamma_{0} \neq 0 .
$$

Then, the following continuous observability inequality holds true: there exists $C_{T}>0$ such that

$$
\int_{0}^{T} \int_{\Gamma_{1}}\left|w_{t}\right|^{2} d \Sigma_{1} \geq C_{T}\left\|w_{0}\right\|_{H_{\Gamma_{0}}^{1}(\Omega)}^{2} .
$$

[This is the C.O.I. (0.3), as specialized to the present Neumann case (8.16).]

For global uniqueness results such as the ones for $\psi$ satisfying either (8.13) or (8.16), we refer to [L-T-Z.2-3].

In the statement of Theorem 8.4, key to the elimination of geometrical conditions on the controlled/observed (and also stabilized) portion $\Gamma_{1}$ of the boundary $\Gamma=\partial \Omega$ is a lemma such as Lemma 6.5.3 for a solution $w$ of (8.1) in the class (8.4): see [Tr.2, Theorem 2.1.4], [T-Y.2, Lemma 7.2], which, in fact, can be reinforced [L-T-Z.2]. As a result of this lemma and of Theorem 8.2, one obtains the following main estimate (at the energy level).

THEOREM 8.5. Assume the hypotheses and the setting of Theorem 8.2. Let $f=0$. Let $w$ be a solution of (8.1) in the class (8.4).

(a) Then the following estimate holds true: there exists a constant $k_{\phi, \tau}>0$ for $\tau_{0}$ sufficiently large, $\tau \geq \tau_{0}>0$, such that, for any $\epsilon_{0}>0$ :

$$
\begin{aligned}
\int_{0}^{T} \int_{\Gamma}\left[\left|\frac{\partial w}{\partial n}\right|^{2}\right. & \left.+\left|w_{t}\right|^{2}\right] d \Sigma+c_{\phi, \epsilon_{0}}\|w\|_{L_{2}\left(0, T ; H^{\frac{1}{2}+\epsilon_{0}}(\Omega)\right)}^{2} \\
& \geq k_{\phi, \tau_{0}}[E(T)+E(0)] .
\end{aligned}
$$

(b) Assume, further, that the solution $w$ satisfies also the conditions of Theorem 8.2(b), that is, $\left.w\right|_{\Sigma_{0}} \equiv 0$ with $\Gamma_{0}$ defined by (6.5.1), then estimate (8.18) holds true with $\int_{\Gamma}$ replaced by $\int_{\Gamma_{1}}$.

Exact controllability. By duality, Theorems 8.3 and 8.4 give exact controllability results of the Schrödinger equation, Eqn. (8.1), (8.3) on the 
Riemannian manifold $M$ on any $T>0$, with controls $L_{2}\left(0, T ; L_{2}\left(\Gamma_{1}\right)\right)$ on the space of optimal regularity $H^{-1}(\Omega)$ [Dirichlet case], or on the space of finite energy $H_{\Gamma_{0}}^{1}(\Omega)$ [Neumann case]. This is a parallel treatment of the case for second-order hyperbolic equations, Theorem 6.5.4 and Theorem 6.5.5. A counterpart of Remark 6.5.1 regarding the passage from $H^{2,2}(Q)$ solutions to $H^{1,1}(Q)$-solutions in the Dirichlet and Neumann cases still holds true for Schrödinger equation [L-T-Z.2].

Uniform stabilization. Neumann case. Consider the following Neumann feedback problem with $\Gamma_{0} \neq \phi$ :

$$
\begin{cases}i w_{t}+\Delta_{g} w=0 & \text { in } Q \\ w(0, \cdot)=w_{0} & \text { in } \Omega \\ \left.w\right|_{\Sigma_{0}} \equiv 0 ; \frac{\partial w}{\partial n}=-w_{t} & \text { in } \Sigma_{i}\end{cases}
$$

The problem is well-posed, in the semigroup sense in $H^{1}(\Omega)$. Then, inequality (8.18) permits to obtain a uniform stabilization result for the Neumann feedback problem (8.19), and with no geometric conditions imposed on the controlled boundary $\Gamma_{1}$.

TheOrEm 8.6. Assume $\left(H .1^{\prime}\right)=(3.1 .2)$ and $(A .1)=(8.3),($ A.2) $=$ (8.9), and the unique continuation property of Theorem 8.4 with $\Gamma_{0}$ defined by (6.5.1). Then:

(a) For all $\tau>0$ sufficiently large, $\tau \geq \tau_{0}>0$, there exists a constant $k_{\phi, \tau_{0}}>0$ such that

$$
\int_{0}^{T} \int_{\Gamma_{1}}\left|\frac{\partial w}{\partial n}\right|^{2} d \Sigma_{1} \geq k_{\phi, \tau_{0}} E(T) .
$$

[This is the U.S.I. (0.5), as specialized to the present Neumann case.]

(b) Equivalently, there exist constants $M \geq 1, \mu>0$, such that the energy (8.2) of problem (8.19) satisfies

$$
E(t) \leq M e^{-\mu t} E(0), \quad \forall t \geq 0 .
$$

For uniform stabilization under Dirichlet feedback we refer to [L-T.8], [L-T-Z.2-3].

9. Schrödinger equations: Energy method yielding Carleman/ control estimates with no lower-order terms. Purely Neumann B.C. Global unique continuation. So far, the program encompassed by the title of this subsection has been accomplished only in the Euclidean setting; that is, for Eqn. (8.1) with $\Delta_{g}=\Delta\left(\right.$ or $\left.g_{i j}=\delta_{i j}\right)$ [L-T-Z.2]. As for other evolution equations, analysis of the Euclidean case with $\Delta_{g}=\Delta$ is the first step of the investigative process. Once the appropriate approach has been found and the resulting technicalities have been resolved at the 
Euclidean level, then the next step is to extend these approaches and techniques to the Riemannian setting, by replacing the Euclidean metric with the Riemannian metric $g$.

Paper [L-T-Z.2] is, in a sense, the 'perfect' counterpart in the Schrödinger case of the treatment for second-order equations in Section 7, as specialized to the Euclidean setting $\Delta_{g}=\Delta$ (that is, the 'perfect' counterpart of [L-T-Z.1]). The two evolutions - the second-order hyperbolic Eqn. (6.1.1) and the Schrödinger Eqn. (8.1) require vastly different technical treatments, while sharing a common philosophy. Since only the Euclidean case $\Delta_{g}=\Delta$ has been worked out so far (summer 2002), we shall confine ourselves to give here explicitly only the first version of the corresponding Carleman estimate. More results are in [L-T-Z.2], [L-T-Z.3]. Carleman estimates with no lower-order terms are given in the technical paper [Ta.7], in the pseudo-differential setting.

Let $\Omega$ be an open bounded domain in $\mathbb{R}^{n}$, with boundary $\partial \Omega=\Gamma=$ $\overline{\Gamma_{0} \cup \Gamma_{1}}, \Gamma_{0} \cap \Gamma_{1}=\emptyset, \Gamma$ of class $C^{2}$. We consider Eqn. (8.1) in the Euclidean case, that is,

$$
\begin{gathered}
i w_{t}+\Delta w=F(w)+f \text { in } Q=(0, T] \times \Omega, f \in L_{2}(Q), \\
F(w)=q_{1}(t, x) \cdot \nabla w+q_{0}(t, x) w
\end{gathered}
$$

with $\left|q_{1}\right|, q_{0} \in L_{\infty}(Q)$ so that the following pointwise estimate holds true:

$$
|F(w)|^{2} \leq c_{T}\left[|\nabla w|^{2}+|w|^{2}\right], \quad \forall(t, x) \in Q .
$$

This is the setting of Section 8.1, as restricted to the Euclidean case. As in the case of Section 7, the emphasis of [L-T-Z.2] is on the most challenging case: the purely Neumann B.C. case: i.e., Eqn. (9.1) with initial condition $w_{0} \in H^{1}(\Omega)$, and B.C.

$$
\left.\frac{\partial w}{\partial n}\right|_{\Sigma}=0 \quad \text { in } \Sigma=(0, T] \times \Gamma .
$$

Again, the point is that we seek the C.O.I. such as (0.3) or the U.S.I. such as (0.5), involving only the subportion $\Gamma_{1}$ of $\Gamma$. This corresponds to Neumanncontrol on $\Gamma_{1}$ and homogeneous Neumann B.C. on $\Gamma_{0}$. The following assumptions yield Carleman estimates for solutions of (9.1), with no B.C.

(A.1) There exists a strictly convex (non-negative) function $d: \bar{\Omega} \Rightarrow \mathbb{R}$ of class $C^{3}(\bar{\Omega})$, so that the (symmetric) Hessian matrix $\mathcal{H}_{d}$ of $d(x)$ [i.e., the Jacobian matrix of $h(x) \equiv \nabla d(x)]$ is strictly positive on $\bar{\Omega}: \mathcal{H}_{d} \geq \rho I$, $\rho>0$, on $\bar{\Omega}$.

(A.2) [This can, in fact, be removed eventually as in [L-T-Z.1], as described in Section 7, Remark 7.1.]

$$
\inf _{\Omega}|\nabla d(x)|=p>0
$$


We next introduce the same function $\phi(x, t)$ defined in (3.2.1) with $v$ replaced by $d$ and with $T>0$ arbitrary and $c=c_{T}$ sufficiently large as in (3.2.3), so that properties (3.2.4) and (3.2.5) hold true. We define

$$
\mathbb{E}(t)=\int_{\Omega}\left[|\nabla w(t)|^{2}+|w(t)|^{2}\right] d \Omega
$$

\section{Carleman estimates.}

THEOREM 9.1. (first version) Assume (A.1) and (A.2) $=(9.5)$ as well as the standing assumption (9.3) on $F(w)$ and (9.1) on $f$. Let $T>0$, and let $\phi(t, x)$ be defined by (3.2.1) with $v$ replaced by $d$. Let $w$ be a solution of (9.1) [with no B.C.] in the following class

$$
\left\{\begin{array}{l}
w \in C\left([0, T] ; H^{1}(\Omega)\right) ; \quad \nabla_{\tan } w \quad \frac{\partial w}{\partial \nu} \in L_{2}\left(0, T ; L_{2}(\Gamma)\right) \\
w_{t} \in L_{2}\left(0, T ; H^{-1}(\Gamma)\right)
\end{array}\right.
$$

Then: $\tau>0$ :

(i) the following one-parameter family of estimates holds true for all

$$
\begin{aligned}
B_{\Sigma}(w)+ & 4 \int_{0}^{T} \int_{\Omega} e^{2 \tau \phi}|f|^{2} d \Omega d t \\
\geq & \text { const }_{\tau} \int_{0}^{T} \int_{\Omega} e^{2 \tau \phi}|\nabla w|^{2} d \Omega d t+\left[4 \tau^{3} \rho p+\mathcal{O}\left(\tau^{2}\right)-4\right] \\
& \times \int_{0}^{T} \int_{\Omega} e^{2 \tau \phi}|w|^{2} d \Omega d t-c_{T} \tau e^{-2 \tau \delta}[\mathbb{E}(T)+\mathbb{E}(0)] \\
\geq & {\left[\tau \rho p-\frac{9}{2} c_{T}\right] e^{-\delta \tau} \int_{t_{0}}^{t_{1}} \mathbb{E}(t) d t-c_{T} \tau e^{-2 \tau \delta}[\mathbb{E}(T)+\mathbb{E}(0)] } \\
& +\left[4 \tau^{3} \rho p+\mathcal{O}\left(\tau^{2}\right)-4\right] \int_{0}^{T} \int_{\Omega} e^{2 \tau \phi}|w|^{2} d \Omega d t
\end{aligned}
$$

(ii) For all $\tau$ sufficiently large that $\left[4 \tau^{3} \rho p+\mathcal{O}\left(\tau^{2}\right)-4\right] \geq \tau_{0}>0$, we then have

$$
\begin{aligned}
B_{\Sigma}(w) & +4 \int_{0}^{T} \int_{\Omega} e^{2 \tau \phi}|f|^{2} d \Omega d t \\
& \geq \text { const }_{\tau, p, \rho} e^{-\delta \tau} \int_{t_{0}}^{t_{1}} \mathbb{E}(t) d t-c_{T} \tau e^{-2 \tau \delta}[\mathbb{E}(T)+\mathbb{E}(0)],
\end{aligned}
$$

with $c_{\tau, p, \rho} \nearrow \infty$ as $\tau \nearrow \infty$. In both (9.10) and (9.11), the boundary terms $B_{\Sigma}(w)$ are defined (with $h=\nabla d$ ) by: 


$$
\begin{aligned}
B_{\Sigma}(w)= & 2 \int_{0}^{T} \int_{\Gamma} e^{2 \tau \phi}\left[2 \tau^{2}|h|^{2}\right]|w|^{2} h \cdot \nu d \Gamma d t \\
& -2 \int_{0}^{T} \int_{\Gamma} e^{2 \tau \phi} 2 c \tau\left(t-\frac{T}{2}\right)\left[\eta \frac{\partial \xi}{\partial \nu}-\xi \frac{\partial \eta}{\partial \nu}\right] d \Gamma d t \\
& -2 \int_{0}^{T} \int_{\Gamma} e^{2 \tau \phi}\left[\xi_{t} \eta-\xi \eta_{t}\right] \tau h \cdot \nu d \Gamma d t \\
& +\int_{0}^{T} \int_{\Gamma} e^{2 \tau \phi}\left[2 \tau^{2}|h|^{2}-\tau \Delta d\right]\left[\bar{w} \frac{\partial w}{\partial \nu}+w \frac{\partial \bar{w}}{\partial \nu}\right] d \Gamma d t \\
& +2 \int_{0}^{T} \int_{\Gamma} e^{2 \tau \phi} \tau h \cdot\left[\nabla \bar{w} \frac{\partial w}{\partial \nu}+\nabla w \frac{\partial \bar{w}}{\partial \nu}\right] d \Gamma d t \\
& -2 \int_{0}^{T} \int_{\Gamma} e^{2 \tau \phi}|\nabla w|^{2} \tau h \cdot \nu d \Gamma d t
\end{aligned}
$$

where $\xi=\operatorname{Re} w$ and $\eta=\operatorname{Im} w$.

As usual, establishing the first Carleman estimate is a main part of the entire proof leading to control-theoretic results. The critical novelty of Eqn. (9.10) over Eqn. (8.6) is that in (9.10) the lower-order term (last integral term) comes with a positive coefficient, for $\tau$ sufficiently large, and hence can be absorbed within the $\mathbb{E}(t)$-term. We then accrue the same benefits as in Section 7. The second (final) Carleman estimate (under further assumptions (8.9) as in Section 8) also comes with no lower-order terms, as do the C.O.I. and the U.S.I., whereby one then obtains global unique continuation results and control-theoretic inequalities in one shot, as part of the same flow of arguments. We refer to [L-T-Z.2] for more details. Such work with $\Delta_{g}=\Delta$ should form the basis of a generalization to the Riemannian setting, in the same way as the Euclidean paper [LT-Z.1] led to the Riemannian paper [T-Y.2] for second-order hyperbolic equations.

10. Plate equations on the Riemannian manifold: Energy methods in the Riemannian metric yielding Carleman/control estimates with lower-order terms.

Model. Assumptions. We use the same notation of Sections 6, 7, 8. The philosophical strategy is the same as that of Sections 6 or 8 . Accordingly, our exposition will be concise.

In this section we study the following plate equation, of Euler-Bernoulli type, on a bounded set $\Omega$ of the Riemannian manifold $M$, with LaplaceBeltrami operator $\Delta_{g}$ :

$$
w_{t t}+\Delta_{g}^{2} w+F(w)+f=0 \quad \text { in }(0, T] \times \Omega \equiv Q, \quad f \in L_{2}(Q)
$$


where $\Delta_{g}$ is the Laplace-Beltrami operator on $M$. For this equation, the 'energy level' is $H^{3}(\Omega) \times H^{1}(\Omega)$ for $\left\{w, w_{t}\right\}$. We define

$$
E(t) \equiv \int_{\Omega}\left[|D(\Delta w)|^{2}+\left|D w_{t}\right|^{2}\right] d \Omega .
$$

Accordingly, we make the following assumptions on the energy level differential term:

(A.1): $F(w)$ satisfies the following estimate

$(10.3)|F(w)|^{2} \leq c_{T}\left\{|D w|^{3}+\left|D^{2} w\right|^{2}+|D w|^{2}+w^{2}+\left|D w_{t}\right|^{2}+w_{t}^{2}\right\}, \forall t, x \in Q$.

A remark such as Remark 6.1.1 applies. Furthermore, we assume $f \in L_{2}(Q)$.

Strictly convex function $\boldsymbol{v}(\boldsymbol{x})$. As in Sections $6.2,7$, and 8, we assume hypothesis $($ H.1 $)=(3.1 .1)$ of Section 3.1: there exists a strictly convex (non-negative) $C^{2}$-function $v: \bar{\Omega} \Rightarrow$, so that (3.1.1) holds true.

We then define the same pseudo-convex function $\phi(x, t)$ as in (3.2.1), however, for $T>0$ arbitrary and $c=c_{T}$ such that $4 \sup _{\Omega} d(x) \leq c T^{2}$, precisely as in the case of the Schrödinger equations in Section 8. Thus, properties (i) and (ii) in (3.2.4) and (3.2.5) continue to hold true with these choices. As in Section 6.3, we see that model (10.1) includes the case of a plate-like equation of Euler-Bernoulli type with variable coefficients defined on an open bounded domain $\tilde{\Omega}$ of $\mathbb{R}^{n}: w_{t t}+\mathcal{A}^{2}(w)+F(w)+f=0$ on $(0, T] \times \tilde{\Omega}$, where $\mathcal{A}$ is the second-order elliptic differential operator defined by $(6.3 .1)$.

The results below were obtained in [L-T.14] in the Euclidean case $\Delta_{g}=\Delta\left(g_{i j}=\delta_{i j}\right)$ and generalized in [L-T-Y.4] to the Riemannian setting. Related results are in [Ta.1-3] in the pseudo-differential setting.

Carleman estimates with lower-order terms. The present pattern repeats that of Sections 6 and 8 .

THEOREM 10.1. [L-T.14], [L-T-Y.4] (Carleman estimate, first version) With reference to (10.1), assume $(A .1)=(10.3)$ on the energy level term $F(w) ;(H .1)=(6.2 .1)$ on the strictly convex function $v(x)$; and (10.1) on $f$. Let $w$ be a solution of (10.1) in the following class

$$
\left\{\begin{array}{l}
\left\{w, w_{t}\right\} \in L_{2}\left(0, T ; H^{3}(\Omega) \times H^{1}(\Omega)\right) \\
w_{t}, \Delta_{g} w, \frac{\partial \Delta_{g} w}{\partial n}, D w_{t}, D\left(\Delta_{g} w\right) \in L_{2}(\Sigma)
\end{array}\right.
$$

Let $\phi(x, t)$ be the function defined in (3.2.1), this time with $T>0$ arbitrary and $c=c_{T}$ sufficiently large as to have $4 \sup _{\Omega} v(x) \leq c T^{2}$. Let $E(t)$ be defined by (10.2). Then, for all values $\tau>0$, the following oneparameter family of estimates holds true:

$$
B_{\Sigma}(w)+\frac{2}{\tau} \int_{Q} e^{\tau \phi} f^{2} d Q+C_{\phi, \tau} \max _{[0, T]}\left\{\int_{\Omega}\left[\left|D^{2} w\right|^{2}+|D w|^{2}+w^{2}+w_{t}^{2}\right] d \Omega\right\}
$$




$$
\begin{aligned}
& \geq\left(\rho-\frac{C}{\tau}\right) \int_{Q} e^{\tau \phi}\left[\left|D w_{t}\right|^{2}+\left|D\left(\Delta_{g} w\right)\right|^{2}\right] d Q-C \tau e^{-\delta \tau}[E(T)+E(0)] \\
& \geq\left(\rho-\frac{C}{\tau}\right) e^{-\frac{\tau \delta}{2}} \int_{t_{0}}^{t_{1}} E(t) d t-C \tau e^{-\delta \tau}[E(T)+E(0)]
\end{aligned}
$$

where the boundary terms $B_{\Sigma}(w)$ on $(0, T] \times \Gamma=\Sigma$ are given by

$$
\begin{aligned}
B_{\Sigma}(w) \equiv & B_{\Sigma}^{1}(w)+B_{\Sigma}^{2}(w) ; \\
B_{\Sigma}^{1}(w)= & \int_{\Sigma}\left\{\left[H\left(w_{t}\right)+w_{t} \operatorname{div} H\right] \frac{\partial w_{t}}{\partial n}+H\left(\Delta_{g} w\right) \frac{\partial \Delta_{g} w}{\partial n}\right\} d \Sigma \\
& -\int_{\Sigma}\left\{\left[w_{t} \Delta_{g} w_{t}+\frac{1}{2}\left(\left|D w_{t}\right|^{2}+\left|D\left(\Delta_{g} w\right)\right|^{2}\right)\right]\langle H, n\rangle\right. \\
& \left.+\frac{1}{2} w_{t}^{2}\langle D(\operatorname{div} H), n\rangle\right\} d \Sigma ; \\
B_{\Sigma}^{2} \equiv & \frac{1}{2} \int_{\Sigma}\left\{\left[\Delta_{g} w \frac{\partial \Delta_{g} w}{\partial n}-w_{t} \frac{\partial w_{t}}{\partial n}\right] \operatorname{div} H\right. \\
& \left.+\frac{1}{2}\left[w_{t}^{2}-\left.\left(\Delta_{g} w\right)\right|^{2}\right]\langle D(\operatorname{div} H), n\rangle\right\} d \Sigma,
\end{aligned}
$$

where $H$ is a vector field $H=e^{\tau \phi} D v$.

Remark 10.1. (Idea of proof) [L-T-Y.4] The proof of Theorem 10.1 is by energy methods in the Riemannian metric: it uses the following main multipliers, where $D v=D \phi$ :

$$
H\left(\Delta_{g} w\right)=e^{\tau \phi(x, t)} D v\left(\Delta_{g} w\right), \quad w \operatorname{div} H=\operatorname{div}\left(e^{\tau \phi} D v\right) w .
$$

The multipliers (10.10) represent a far-reaching generalization of the energy methods (multipliers) used for the pure Euclidean Euler-Bernoulli equation in the late '80s [L-T.2], [L-T.4-5], [L.1]. Historical considerations similar to those already made for second-order hyperbolic equations, Eqn. (6.1.1), in Remark 6.4.1 and for the Schrödinger Eqn. (8.1), in Remark 8.1, apply now regarding the multipliers (10.10) in connection with the Euler-Bernoulli equation (10.1), (10.3). More precisely,

(1) If (i) Eqn. (10.1) is defined on a Euclidean domain $\Omega$ (thus $\Delta_{g}=$ $\Delta=$ Euclidean Laplacian, $g_{i j}=\delta_{i j}$ ), and, moreover, (ii) $F(w) \equiv 0$ (no energy level terms), then we recover the 'classical' multipliers $h \cdot \nabla \Delta w$ and $\Delta w$ div $h$ of [L-T.2], [L-T.4-5] with $h(x)$ a coercive field in $\Omega$ (or the special case of [L.1] with a radial field $\left.h(x)=\left(x-x_{0}\right)\right)$, by taking in (10.10) the Euclidean topology and setting $\tau=0$.

(2) Generalizing case (i), if in (10.1) we still have $\Delta_{g}=\Delta$, but $F(w)$ is now an energy level operator satisfying the Euclidean version of (10.3), then we recover the multipliers used in [L-T.14], by taking the Euclidean metric in (10.10). 
It was the case in [L-T.14] that prompted the multipliers (10.10) for the general case (10.1), (10.3), under the inspiration of previously covered cases: second-order hyperbolic equations (Remark 6.4.1), and Schrödinger equations (Remark 8.1). The proof in [L-T-Y.4] for the general case is a parallel development of that in [L-T.14]: computations in the Euclidean metric performed in [L-T.14] are replaced by counterpart computations in the Riemannian metric $g$, using the Levi-Civita connection $D$.

This time, as in the case of Schrödinger equations see $(\mathrm{A} .2)=(8.9)$ in Section 8, and unlike the case of second-order hyperbolic equations in Section 6 , we need to impose a structural assumption on the energy level term $F(w)$. More precisely:

(A.2):

(10.11) Assume now that $F(w)$ is such that inequality (3.2.7) holds true,

with $E(t)$ defined by (10.2). See [L-T-Y.4], [Hor.3].

We then have, as a consequence of $(\mathrm{A} .2)=(10.11)$ used in Theorem 10.1:

THEOREM 10.2. [L-T.14], [L-T-Y.4] (Carleman estimate, second version) Assume the hypotheses and setting of Theorem 10.1. In addition, assume $\left(\right.$ A.2) $=(10.11)$. Then, for all $\tau>0$ sufficiently large, $\tau \geq \tau_{0}>0$, there exists a constant $k_{\phi, \tau}>0$, such that the following one-parameter family of estimates holds true:

$$
\begin{aligned}
& {\left[1+C_{T}\left(t_{1}-t_{0}\right)\right] B_{\Sigma}(w)} \\
& +C_{T}\left(t_{1}-t_{0}\right)\left\{B T_{e}(w)+\int_{0}^{T}\|f\|_{H^{1}(\Omega)}^{2} d t+\operatorname{lot}(w)\right\} \\
& +\frac{2}{\tau} \int_{Q} e^{\tau \phi} f^{2} d Q+C \tau e^{-\delta \tau} \max _{[0, T]} \int_{\Omega}\left[\left|D^{2} w\right|^{2}+|D w|^{2}+w^{2}+w_{t}^{2}\right] d \Omega \\
& \geq k_{\phi, \tau_{0}}[E(T)+E(0)],
\end{aligned}
$$

where the boundary terms $B_{\Sigma}(w)$ and $B T_{e}(w)$ are defined by (10.8)-(10.10) and (3.2.7).

Control-theoretic results for (10.1), (10.3). The above Carleman estimates at the $H^{3}(\Omega) \times H^{1}(\Omega)$-level - in particular, estimate (10.12) of Theorem 10.2 - form the key basic ground for obtaining continuous observability inequalities (hence, by duality, exact controllability results)/ stabilization inequalities for Eqn. (10.1), when this equation is accompanied by suitable boundary conditions (B.C.)

The $H^{3}(\Omega) \times H^{1}(\Omega)$-level of the preceding estimates are most directly of use when equation (10.1) is supplemented by hinged B.C. However, a full, sharp account even in the case of hinged B.C.- and surely more so for other B.C. such as clamped B.C.- requires additional tools [L-T.4-5], [L-T.14], [Ta.1-3] to deal with a few remaining issues. These include: (i) the issue of 
reducing the number of traces (see Remark 10.3) needed in the continuous observability estimates (that is, the issue of reducing the number of controls needed to obtain the corresponding (dual) exact controllability results); (ii) the issue of shifting (in particular, shifting down) the $H^{3}(\Omega) \times H^{1}(\Omega)$-level of topologies of the original estimate (10.12) [which is good, as we said, for hinged B.C.]. This shifting is required in order to obtain continuous observability estimates (exact controllability results) also in the case of clamped B.C. While lack of space induces us to leave this program and refer to the aforementioned references, we here derive the corresponding continuous observability/stabilization estimate for hinged B.C., in the case of two traces/two controls), since this follows readily from (10.12). More general consequences will be derived in a subsequent paper.

Uniqueness assumption. Below we shall need the following uniqueness property for an over-determined problem associated with (10.1): Let $w$ be a sufficiently smooth solution of (10.1) as in (10.4) which satisfies the following B.C.

$$
\begin{array}{cc}
\left.w\right|_{\Sigma}=\left.\Delta_{g} w\right|_{\Sigma} \equiv 0 & \text { on }(0, T] \times \Gamma \equiv \Sigma \\
\left.\frac{\partial w}{\partial n}\right|_{\Sigma_{1}}=\left.\frac{\partial \Delta_{g} w}{\partial n}\right|_{\Sigma_{1}} \equiv 0 & \text { on }(0, T] \times \Gamma_{1} \equiv \Sigma_{1},
\end{array}
$$

where the geometrical condition $(6.5 .1)$ or $(6.4 .7)$ for $\Gamma_{0}$ :

$$
\frac{\partial v}{\partial n}=\langle D v, n\rangle \leq 0, \quad x \in \Gamma_{0}
$$

holds true on $\Gamma_{0}=\Gamma / \Gamma_{1}$. Then, in fact: $w \equiv 0$ on $Q=(0, T] \times \Omega$.

We point out that our fourth-order operator is the iteration of a secondorder operator.

Remark 10.2. The following are a few known cases, where the unique continuation property required above for problem (10.1), (10.3) holds true, say, in the Euclidean setting of Section 6.3 , for $w_{t t}+\mathcal{A}^{2} w+F(w)=0$ with variable coefficients, on a bounded domain $\Omega \subset \mathbb{R}^{n}$ :

(1) The case where the coefficients $a_{i j}(x)$ of the elliptic operator $\mathcal{A}$ in (6.3.1) satisfy $a_{i j}(x) \in C^{1}(\Omega)$, while the coefficients $q_{\alpha}$ and $r_{\alpha}$ of the energy level term $F$ in (10.3) are time-independent and in $L_{\infty}(\Omega)$ in the space variable.

Indeed, in this case, the unique continuation property for the dynamical problem (10.1), (10.3) can be converted (via Laplace transform) into a unique continuation property for the corresponding fourth-order elliptic problem with space-variable coefficients. This latter problem has all four boundary conditions (Cauchy data) zero on the portion $\Gamma_{1}$ of the boundary. As a consequence, the solution of the corresponding elliptic problem has to vanish in a suitable neighborhood of $\Gamma_{1}$, interior to $\Omega$. At this point, we can apply Hörmander's 1959-theorem [Hor.2] (of which a new proof has been recently given in [E.1, Theorem 1.1]) and conclude that, then, the solution 
of such elliptic problem must vanish on all of $\Omega$ : $w \equiv 0$ in $\Omega$, as desired. [A stronger version of the unique continuation result in [Hor.2] for certain fourth-order elliptic equations, which are iterations of two second-order elliptic equations, is given in [S.1], under "weaker vanishing requirements," beyond our present needs. Papers [Hor.2] and [S.1] improve upon a prior result in [P.1], where the fourth-order equation has principal part restricted to $\Delta^{2}$, followed by all third-order terms.]

(2) The case -given in [I.1, Theorem 5.1, p. 137] - of the equation in a Euclidean setting as in Section 6.3, where, however, the differential operator $\mathcal{A}=\Delta$ in (6.3.1) $\left[a_{i j}=\delta_{i j}\right]$, but with coefficients of $F$ allowed to vary in both time and space:

$$
F(w)=\sum_{|\beta| \leq 1} a_{\beta} \partial^{\beta} \Delta w+\sum_{|\alpha| \leq 2} a_{\alpha} \partial^{\alpha} w,
$$

$a_{\alpha} \in L(Q), Q=(0, T) \times \Omega$, plus some additional conditions. This result in [I.1] requires, however, all four zero B.C. on the entire boundary $\Gamma$ (so, in (10.14), we must take $\Gamma_{1}=\Gamma$ ). We remark that a readjustment of the proof for unique continuation result given in [E-L-T.1, Section 10], [E-LT.2] for the corresponding Kirchhoff plate with all four B.C. zero, but this time only on an arbitrary common portion of the boundary, of positive measure, is likely to admit (mutatis mutandis) a counterpart version to the Euler-Bernoulli plate. [The constant $\gamma>0$ for the Kirchhoff plate, which accounts for rotational inertia, becomes $\gamma=0$ on the Euler-Bernoulli plate.] Should this be the case, the required $\Gamma_{1} \subset \Gamma$ in (10.14) subject to (10.15) would be allowed. The unique continuation result in [E-L-T.1, Section 10], [E-L-T.2] for the Kirchhoff equation with all four zero B.C. on an arbitrary portion of the boundary extended the prior result [I.1, Theorem 1.2, p. 136], where vanishing of all four B.C. on the entire boundary was required.

Continuous observability inequality.

ThEOREM 10.3. With reference to Eqn. (10.1) with $f=0$, assume $(A .1)=(10.3)$ on $F$ and $(H .1)=$ (3.1.1). In addition, assume: (i) the hinged boundary condition (10.13) on all of $\Sigma=(0, T] \times \Gamma$, as well as (ii) the geometrical condition (10.15) on $\Gamma_{0}$.

(a) Then, given $T>0$, there exists $C_{T}>0$, such that the following estimate holds true:

$$
\int_{0}^{T} \int_{\Gamma_{1}}\left[\left(\frac{\partial w_{t}}{\partial n}\right)^{2}+\left(\frac{\partial \Delta w}{\partial n}\right)^{2}\right] d \Gamma_{1} d t+\operatorname{lot}(w) \geq C_{T}[E(0)+E(T)]
$$

(b) Assume further the uniqueness property stated before: (10.1), (10.13), (10.14), imply $w \equiv 0$ on $Q$. Then, estimate (10.16) simplifies to the following Continuous Observability Inequality:

$$
\int_{0}^{T} \int_{\Gamma_{1}}\left[\left(\frac{\partial w_{t}}{\partial n}\right)^{2}+\left(\frac{\partial \Delta w}{\partial n}\right)^{2}\right] d \Gamma_{1} d t \geq c_{T}[E(0)+E(T)]
$$


Remark 10.3. It is possible to reduce the number of traces arising in the C.O.I. or U.S.I. of plate equations. This topic - which is algebraic or analytic rather than geometric - will take us too far afield. In the case of the C.O.I. in the hinged case above, an ad hoc method was given in [Le.2] and is reproduced in [K.1]. In the more challenging stabilization problem of an Euler-Bernoulli plate with (true) "physical moment B.C. (rather than simply $\left.\Delta w\right|_{\Sigma}$ ), [Las-Hor.1] also succeed in eliminating a trace from the U.S.I. A general algebraic method for expressing a trace in terms of others, rooted in PDE theory [Tay.1], was given in [Ta.1] for general evolution equation in the pseudo-differential language. Manifestations of it can be found in [L-T.14] for various single PDE classes, and in [Las.3] for the boundary stabilization of the 3-d structural acoustic model. At any rate, the Riemannian energy method of the present Part III merges well with the techniques of eliminating superfluous traces in the final C.O.I. and U.S.I. for plates.

Exact controllability. As is well-known [L-T.2,4-5], by duality, the continuous observability inequality (10.17) is equivalent to the property of exact controllability of the corresponding non-homogeneous boundary control problem, with controls in the hinged B.C., in the space of 'optimal regularity' [L-T.2,4-5], [L.1].

Remark 10.4. The approach of Section 9 on Schrödinger equations accrues a benefit also in the case of the Euler-Bernoulli equation in the Euclidean case (i.e., Eqn. (10.1) with $\Delta_{g}=\Delta, g_{i j}=\delta_{i j}$, and $F(w) \equiv 0$ ) with hinged B.C. In fact, this case can be rewritten as the iteration of two pure Schrödinger equations, with Dirichlet B.C., to which the results of Section 9 can be applied. The result of this approach is that the constant $c_{T}$ in (10.17) is thus explicit, contrary to the classical results [L-T.2, 45], [L.1], etc., where it is not explicit, due to the compactness uniqueness contradiction argument to absorb l.o.t. $(w)$.

Part IV: A dynamic shell model.

11. Uniform stabilization of a shallow shell model with nonlinear boundary feedbacks. Critical marriage between Riemannian geometry (continuous observability inequality) and microlocal analysis (boundary trace estimates). A dynamic shell: The need for differential geometry. A shell is a body in $\mathbb{R}^{3}$. We think of the middle surface of the shell as occupying a bounded region $\Omega$ of a smooth orientable surface $M$ in $\mathbb{R}^{3}$. Then, the shell of thickness $h>0$ ("small") is defined by

$$
S=\left\{p: p=x+z N(x), \quad x \in \Omega,-\frac{h}{2}<z<\frac{h}{2}\right\} .
$$

Here $N(x)$ is the unit normal field on $\Omega$. A dynamic model of a shell describes its displacement as a function of time. The displacement is a 
3-dimensional vector $\{W, w\}$. Here, $W$ is the 2-dimensional in-plane displacement, defined on the tangent plane of each point $x \in \Omega$; instead, $w$ is the scalar normal displacement. The mathematical model of a dynamic shell consists of two coupled variable coefficient Partial Differential Equations (PDEs), both of hyperbolic type: a 2-d system of elasticity for the in-plane displacement $W$ and a plate-like equation of Kirchhoff type for the scalar normal displacement $w$. The coefficients of the principal part of the operators are variable, due to the curved nature of the shell. Classically, the topic of static shells is covered by many books. They all assume the middle surface of a shell to be described by one coordinate patch: this is the image in $\mathbb{R}^{3}$ of a smooth function defined on a connected domain of $\mathbb{R}^{2}$. This view has geometrical limitations, as it forces the exclusion of interesting objects such as a half sphere, or a sphere. Moreover, the classical models use traditional geometry and end up with highly complicated analytical models. In these, the explicit presence of the Christoffel symbols $\Gamma_{i j}^{k}$, make them unsuitable for energy method computations, of the type needed for continuous observability/stabilization estimates, such as (0.3), (0.5), due to the presence of variable coefficients (in space) in the principal part of the differential operators, as well as in the energy level terms. Here, instead, in line with the main aim of the present paper, we shall present a recently proposed [Y.3], [Y.4], intrinsic model of a shallow shell viewed as a 2-d Riemannian manifold, within the intrinsic, coordinate-free setting of differential geometry. This approach allows for the use of a computational energy method in the Riemannian metric, as in Part III for single PDE equations.

Boundary feedback dissipative shell model: Stabilization. In this section, we first present the resulting analytic model of a shell, as it arises when suitable dissipative terms are imposed on the boundary conditions. The choice of the suitable dissipative terms is a non-trivial problem in its own merit, particularly in the case of the physically relevant, but mathematically challenging, free boundary conditions. Here we focus on free boundary conditions, and we then select the boundary feedback dissipative model proposed in [L-T.29]. This augments, with suitably chosen dissipative boundary terms, the original boundary homogeneous model in [Y.3]. We choose the boundary damping terms to be nonlinear. We then present a recent result [L-T.29] which claims that the aforementioned shallow shell model with nonlinear dissipative terms in the free boundary conditions is, in fact, uniformly stable.

Combination of differential geometry and microlocal analysis on traces as critical for the solution of the stabilization problem. The solution of the present stabilization problem relies critically on the combination or fusion of the differential geometric description of the shell - in particular, the continuous observability estimate in [Y.4] given in Section 11.3 below - with a delicate PDE-microlocal analysis, yielding sharp trace regularity of the solutions of the elastic waves (Section 11.5) 
and of the Kirchhoff plates (Section 11.6) - the two components of the displacement of a shell. This way, we first of all solve the problem, and, in the process, we achieve two main benefits: (i) we dispense altogether with restrictive geometrical conditions on the controlled part of the boundary of the shell, of the type used in wave and plate literature [Lag.3]; (ii) we avoid unnatural and mathematically undesirable terms in the boundary feedbacks of the elastic wave [P-T.1] even in the flat case, whose purpose was to cancel out boundary traces, which one would not control without sharp trace theory; the price paid this way was that of injecting boundary terms which are not in $L_{2}$. More explicitly, the sharp, microlocal trace theory of the plate component ( $w$ below in Section 11.6) is not strictly critical for achieving some solution of the present uniform stabilization problem: in fact, one could get a solution at the price of assuming, instead, restrictive and unnecessary geometrical conditions on the controlled part of the boundary $\Gamma_{1}$ as in prior literature [Lag.3]. By contrast, the contribution of a sharp, microlocal trace theory of the elastic wave component $W$ in Section 11.5 is indispensible for the very solution of the present uniform stabilization problem.

11.1. A differential geometric model for a dynamic shallow shell's model in nonlinear, dissipative, feedback form. The middle surface of the shell is a bounded region $\Omega$, which lies on a smooth orientable, two-dimensional surface $M$ of $\mathbb{R}^{3}$. The regular boundary (on $M$ ) of $\Omega$ is denoted by $\Gamma=\Gamma_{0} \cup \Gamma_{1}, \Gamma_{0} \cap \Gamma_{1}=\emptyset$ and consists of two disjoint portions: $\Gamma_{0}$ which will be the 'uncontrolled' part of the boundary; and $\Gamma_{1}$ which will be the 'controlled' part of the boundary; that is, the one where the dissipative feedback is active. The coupled system of two hyperbolic PDEs in the displacement vector $[W, w]$, which represent the dynamic model of a shallow shell in feedback form is [L-T.29],

$$
\begin{aligned}
& \Delta w+(1-\mu) B_{3}(w)=-h_{1}\left(\frac{\partial w_{t}}{\partial n}\right) \quad \text { in } \Sigma_{1, \infty} ; \\
& \left\{\begin{array}{c}
\frac{\partial \Delta w}{\partial n}+(1-\mu) B_{4} w-\gamma \frac{\partial w_{t t}}{\partial n}=-\frac{\partial}{\partial \tau} h_{2}\left(\frac{\partial w_{t}}{\partial \tau}\right) \text { in } \Sigma_{1, \infty} \\
\zeta(0, \cdot) \equiv[W(0, \cdot), w(0, \cdot)] \equiv \zeta_{0}=\left[W_{0}, w_{0}\right] \\
\zeta_{t}(0, \cdot) \equiv\left[W_{t}(0, \cdot), w_{t}(0, \cdot)\right] \equiv \zeta_{1}=\left[W_{1}, w_{1}\right]
\end{array}\right.
\end{aligned}
$$

We notice that model (11.1.1) has homogeneous boundary conditions on $\Gamma_{0}$, and suitable nonlinear dissipative feedback terms on $\Gamma_{1}$ involving tangential and normal components of $W_{t}$ and $w_{t}$ through the nonlinear 
scalar functions $g_{i}(\cdot)$ and $h_{i}(\cdot)$. In the boundary homogeneous case, where the boundary functions are all zero: $g_{1} \equiv g_{2} \equiv h_{1} \equiv h_{2} \equiv 0$, the mixed problem (11.1.1) specializes to the one considered in [Y.1-2]. In the flat case, the feedback problem (11.1.1) reduces to (a special case of) the fully nonlinear von Karman system considered in [Las.5], see also [Las.6]: here, the coupling between the $W$ - and the $w$-equation is via nonlinear unbounded terms in the energy space; instead, problem (11.1.1) in the flat case yields no coupling terms: $\mathcal{F}(w) \equiv 0, \mathcal{G}(W) \equiv 0$.

Essential glossary of notation. Equations (11.1.1a-b). Essentially Eqn. (11.1.1a) is a system of elasticity in $W$, while (11.1.1b) is a Kirchhoff-like plate equation in $w$, both defined on the curved surface $\Omega$. In (11.1.1a), we have that $\Delta_{\mu}$ is a Hodge-Laplace type operator applied to 1-forms (equivalently, vector fields) on $M$ and is defined by: $-\Delta_{\mu}=\frac{1-\mu}{2} \delta d+d \delta$. Here $d$ is the exterior differential and $\delta$ is its formal adjoint. The constant $\mu, 0<\mu<1$ (physically $0<\mu<\frac{1}{2}$ ), is the Poisson's coefficient of the material of the shell. Moreover, $k$ and $H$ are, respectively, the Gaussian curvature and the mean curvature of the shell's middle surface $\Omega$. Furthermore, in $(11.1 .1 \mathrm{a}-\mathrm{b}), \Delta$ is the Laplace-Beltrami operator on the manifold $M$. In addition, the coupling terms $\mathcal{F}(w)$ and $\mathcal{G}(W)$ are first-order differential operators on $w$ and $W$, respectively, whose structure is not essential (see [Y.3]). However, in the flat case, the second fundamental form is zero, and so $H \equiv k \equiv 0$, and these coupling terms vanish $\mathcal{F}(w) \equiv \mathcal{G}(W) \equiv 0$. We thus obtain the (usual) system of elasticity in 2-d and a Kirchhoff plate equation, this time uncoupled. Finally, $\gamma=\frac{h}{12}$, where $h$ is the thickness of the shell.

Boundary equations. (11.1.1d-f) Set preliminarily $\frac{\partial \cdot}{\partial n}=\langle D \cdot$, $n\rangle, \quad \frac{\partial}{\partial \tau}=\langle D \cdot, \tau\rangle$, where $D$ denotes the Levi-Civita connection on $M$ in the induced metric from $\mathbb{R}^{3}$. Finally, the boundary operators $B_{1}, B_{2}, B_{3}$, $B_{4}$ in (11.1.1d-f) are defined by

$$
\left\{\begin{aligned}
B_{1}(W, w) & =(1-\mu) \Upsilon(W, w)(n, n)+\mu(w H-\delta W) \\
B_{2}(W, w) & =(1-\mu) \Upsilon(W, w)(n, \tau) \\
B_{3} & \equiv-D^{2} w(\tau, \tau) \\
B_{4} & \equiv \frac{\partial}{\partial \tau}\left[D^{2} w(\tau, n)\right]+k(x) \frac{\partial w}{\partial n}+\ell w, \ell \geq 0
\end{aligned}\right.
$$

In (11.1.2)-(11.1.5), $\Upsilon(\zeta)=\Upsilon(W, w)$ is the linearized 2-covariant strain tensor defined by $[\mathrm{Y} .3,(1.22)]$

$$
\Upsilon(W, w)=\frac{1}{2}\left(D W+D^{*} W\right)+w \Pi
$$

in terms of the covariant differential $D W$ of $W$ and its transpose $D^{*} W$, as well as of the second fundamental form $\Pi$ of the surface $M$. Moreover, 
$D^{2} w$ is the Hessian of $w\left[-D^{2} w\right.$ denotes the change of curvature tensor of the middle surface $\Omega]$. We note that the operator $B_{4}$ associated with the plate component of the shell is given in terms of normal and tangential coordinates, precisely as in [L-T.24, Chapter 3, Appendix D], which is a more convenient geometric and analytic representation of that arising in the variation model [Lag.2-3]. In (11.1.5), $\ell$ is a non-negative constant, whose role is seen in hypothesis (H.4) below (11.2.18). The present model appears to be the linearization of models by Bernadou and Ciarlet.

11.2. Main results: Well-posedness and uniform stabilization. Well-posedness of feedback problem (11.1.1a-g). The following well-posedness/regularity results are known for the feedback problem $(11.1 .1 \mathrm{a}-\mathrm{g})$.

THEOREM 11.2.1. (a) [generalized (weak) solutions] Assume that: the non-linear functions $h_{i}, g_{i}$ in (11.1.1d-f) are possibly multivalued, monotone, and that $0 \in h_{i}(0), 0 \in g_{i}(0)$. Then: there exists a unique, global solution of finite energy of problem (11.1.1a-g). This is to say that: for any initial data (see [He.1], [L-T.29], [Y.3], [Y.4], for these spaces)

$$
W_{0}, W_{1} \in H_{\Gamma_{0}}^{1}(\Omega, \Lambda) \times L^{2}(\Omega, \Lambda) ; w_{0}, w_{1} \in H_{\Gamma_{0}}^{2}(\Omega) \times H_{\Gamma_{0}}^{1}(\Omega),
$$

that is, subject to the boundary conditions (B.C.) $W_{0}=w_{0}=\frac{\partial w_{0}}{\partial n}=w_{1}=$ 0 on $\Gamma_{0}$, there exists a unique solution

$$
\begin{aligned}
\{W, w\} & \in C\left([0, T] ; H_{\Gamma_{0}}^{1}(\Omega, \Lambda) \times H_{\Gamma_{0}}^{2}(\Omega)\right) ; \\
\left\{W_{t}, w_{t}\right\} & \in C\left([0, T] ; L_{2}(\Omega, \Lambda) \times H_{\Gamma_{0}}^{1}(\Omega)\right),
\end{aligned}
$$

where $T>0$ is arbitrary. This solution is described by a non-linear semigroup acting on the finite energy space. The form of the generator is given in (11.3.3) below.

(b) [regular solutions] Assume that the boundary functions $h_{i}, g_{i}$ satisfy, in addition to the above hypotheses of part (a), the following more specific hypotheses: $h_{i}, g_{i}$ are single valued, and moreover $h_{i}, g_{i} \in C(\mathbb{R})$; $h_{i}^{\prime}, g_{i}^{\prime} \in L_{\infty}(\mathbb{R})$. Then: for any initial data (see [L-T.29], [He.1], [Y.3-4] for these spaces)

$$
W_{0}, W_{1} \in H^{2}(\Omega, \Lambda) \times H^{1}(\Omega, \Lambda) ; w_{0}, w_{1} \in H^{3}(\Omega) \times H^{2}(\Omega),
$$

subject to the B.C. below (11.2.1), there exists a unique solution of (11.1.1a-g):

$$
\begin{aligned}
{[W, w] } & \in C\left([0, T] ; H^{2}(\Omega, \Lambda) \times H^{3}(\Omega)\right) ; \\
{\left[W_{t}, w_{t}\right] } & \in C\left([0, T] ; H^{1}(\Omega, \Lambda) \times H^{2}(\Omega)\right) .
\end{aligned}
$$

Henceforth, the boundary functions $h_{i}, g_{i}$ are assumed to satisfy the condition of Theorem 11.2.1 unless otherwise stated. 
Uniform stabilization. The main goal of the present section is to show that the solutions of problem (11.1.1a-g), asserted by Theorem 11.2.1, decay to zero at $t \rightarrow \infty$ at a uniform rate. Since the dissipative feedback terms are located on the portion $\Gamma_{1}$ of the boundary $\Gamma$ of the mid-surface $\Omega$ on the surface $M$, and the dissipation needs to be propagated from the boundary onto the interior of the shell, then we surmise that the geometry of the shell is bound to play a critical role in the stabilization arguments. Indeed, we shall require geometric assumptions (H.1) and (H.2) below.

Preliminaries. Let $\zeta=[W, w]$ be the displacement field of the middle surface $\Omega$ of the shell and denote $\hat{\zeta}=[\hat{W}, \hat{w}]$. Introduce the bilinear form $[Y .3,(1.25)]$

$$
B(\zeta, \hat{\zeta})=a(\Upsilon(\zeta), \Upsilon(\hat{\zeta}))+\gamma a\left(D^{2} w, D^{2} \hat{w}\right), \quad \gamma=\frac{h^{2}}{12}
$$

See [B-B, p. 15] for (11.2.6). In (11.2.6), the 2-covariant tensor $\Upsilon(\cdot, \cdot)$ was defined by (11.1.6), while the 2-covariant tensor $D^{2} w$ is the Hessian of $w$, which is defined in (3.1.1). Moreover, in $(11.2 .6), a(\cdot, \cdot)$ is a bilinear form $[$ Y.3, (1.26)]

$$
a\left(T_{1}, T_{1}\right)=(1-\mu)\left\langle T_{1}, T_{1}\right\rangle_{T_{x}^{2}}+\mu\left(\operatorname{tr} T_{1}\right)^{2}, x \in \Omega, T_{1} \in T^{2}(\bar{\Omega}),
$$

defined on 2-order tensors $T^{2}(\bar{\Omega})$ of $\bar{\Omega}$, see [Y.3-4], [L-T.29] for the inner product and the trace $t r$. Finally, with $(11.2 .6)$ we can associate the following symmetric bilinear form, defined directly on the middle surface $\Omega$ :

$$
\begin{gathered}
\mathcal{B}(\zeta, \hat{\zeta})=\int_{\Omega} B(\zeta, \hat{\zeta}) d x ; \quad \zeta(x)=W(x)+w(x) N(x) \\
W(x) \in M_{x}, \quad x \in \Omega
\end{gathered}
$$

$M_{x}$ being the tangent space at $x \in M . N(x)$ is the unit normal field.

After these preliminaries, to state our stabilization result, we recall that the energy functional associated with model (11.1.1) is given by

$$
E(t)=E_{k}(t)+E_{p}(t)
$$

where $E_{k}$ is the kinetic energy

$$
\begin{aligned}
E_{k}(t) & \equiv \int_{\Omega}\left\{\left|W_{t}\right|_{T_{x}}^{2}+w_{t}^{2}+\gamma\left|D w_{t}\right|_{T_{x}}^{2}\right\} d x \\
& =\left\|W_{t}\right\|_{L^{2}(\Omega, \Lambda)}^{2}+\left\|w_{t}\right\|_{L^{2}(\Omega)}^{2}+\gamma\left\|D w_{t}\right\|_{L^{2}(\Omega, \Lambda)}^{2}
\end{aligned}
$$

(see [He.1 [Y.3-4], [L-T.29] for these spaces), and $E_{p}$ is the potential energy, see $(11.2 .6)-(11.2 .8)$ :

$$
\begin{aligned}
E_{p}(t) & \equiv \mathcal{B}(\zeta, \zeta)=\int_{\Omega} B(\zeta, \zeta) d x \\
& =\int_{\Omega}\left[a(\Upsilon(W, w), \Upsilon(W, w))+\gamma a\left(D^{2} w, D^{2} w\right)\right] d x, \quad \zeta=[W, w]
\end{aligned}
$$


Next, in line with the statement above (11.2.6), we need to impose some geometric conditions on the shell.

Geometric assumptions. We shall assume the following hypotheses that were needed in [Y.4] to prove the observability estimate which we shall invoke in Section 11.3.

(H.1): ellipticity of the shell strain energy: there exists a constant $\lambda_{0} \geq 1$ such that

$$
\left\{\begin{array}{l}
\lambda_{0} \mathcal{B}(\zeta, \zeta) \geq\|D W\|_{L^{2}\left(\Omega, T^{2}\right)}^{2}+\gamma\left\|D^{2} w\right\|_{L^{2}\left(\Omega, T^{2}\right)}^{2} \\
\text { for } \zeta=[W, w] \in H^{1}(\Omega, \Lambda) \times H^{2}(\Omega),
\end{array}\right.
$$

where the function spaces are defined in [He.1], [Y.3-4], [L-T.29] below.

In particular, a sufficient condition for (H.1) to hold true is that both $\Pi$ and $D \Pi$ are small enough [B-O], where $\Pi$ is the second fundamental form of $M$. A much weaker condition where (H.1) holds true is given in [Y.3, Theorem 3.2]: it basically says that the shell is "sufficiently shallow."

Main assumption (H.2). We assume that there exists a vector field $V \in \mathcal{X}(M)$ such that the covariant differential satisfies

$$
D V(X, X)=b(x)|X|^{2}, \quad X \in M_{x}, x \in \bar{\Omega},
$$

where $b$ is a function on $\Omega$. Set

$$
a(x)=\frac{1}{2}\langle D V, \mathcal{E}\rangle_{T_{x}^{2}}, \quad x \in \bar{\Omega},
$$

where $\mathcal{E}$ is the volume element of $M$. Moreover, suppose that $b$ and $a$ satisfy the following inequality

$$
2 \min _{x \in \bar{\Omega}} b(x)>\lambda_{0}(1+\mu) \max _{x \in \bar{\Omega}}|a(x)| .
$$

Assumption (H.2) consists of (11.2.15) and (11.2.16).

Illustrations where Assumption (H.2) holds true are given in [Y.3]. The include shells whose mid-surface lies on a surface of constant curvature or a surface of revolution.

Theorem 11.2.2. Assume (H.1) in (11.2.14) and (H.2) in (11.2.15), (11.2.16) above. In addition to the well-posedness assumptions on $h_{i}, g_{i}$ in Theorem 11.1.1(a), assume further that:

(H.3): there exist positive constants $0<m<M$ and a sufficiently large constant $R>0$, such that: for all $s \in \mathbb{R}$ with $|s|>R$, we have

$$
m|s|^{2} \leq g_{i}(s) s \leq M|s|^{2} ; m|s|^{2} \leq h_{i}(s) s \leq M|s|^{2}, i=1,2 .
$$

Next, assume that: $\Gamma_{0} \neq \emptyset$.

(H.4): either the coefficient $\ell$ in (11.1.1) is positive: $\ell>0$ or else 
Let $[W, w]$ be a weak solution of the feedback problem (11.1.1a-g), as asserted by Theorem 11.2.1(a). Then: there exists a constant $T_{0}>0$ such that, with reference to the energy $E(t)$ in (11.2.9), the following estimate holds true:

$$
E(t) \leq C(E(0)) s\left(\frac{t}{T_{0}}-1\right), \quad \forall t \geq T_{0},
$$

where $C(E(0))$ denotes a constant depending on the initial energy $E(0)$, and where $s(t)$ is a real-valued function converging to zero: $s(t) \rightarrow 0$ as $t \rightarrow \infty$, which is constructed as a solution of the following Cauchy problem

$$
s_{t}(t)+q(s(t))=0, \quad s(0)=E(0)
$$

involving a non-linear ordinary differential equation where the function $q(\cdot)$ is, in turn, constructed from the data of problem (11.1.1). More precisely, the non-linear monotone increasing function $q(\cdot)$ is determined entirely from the behavior at the origin of the non-linear boundary functions $g_{i}, h_{i}$, according to the following algorithm [L-Ta.1].

Step 1. Due to the assumed monotonicity of the non-linear boundary functions $h_{i}, g_{i}$ one can readily construct [L-Ta.1] functions $\tilde{g}_{i}, \tilde{h}_{i}$, concave and strictly increasing; vanishing at the origin: $\tilde{g}_{i}(0)=\tilde{h}_{i}(0)=0$, such that the following inequalities are satisfied for $|s| \leq 1$ :

$$
\tilde{g}_{i}\left(s g_{i}(s)\right) \geq|s|^{2}+\left|g_{i}(s)\right|^{2} ; \quad \tilde{h}_{i}\left(s h_{i}(s)\right) \geq|s|^{2}+\left|h_{i}(s)\right|^{2}, \quad \forall|s| \leq 1 .
$$

We then define first the functions $r_{0}(\cdot)$ and its rescaled version $r(\cdot)$ by:

$$
r_{0}(s) \equiv \sum_{i=1}^{2} \tilde{g}_{i}(s)+\tilde{h}_{i}(s) ; \quad r(\cdot)=r_{0}\left(\frac{\cdot}{\operatorname{meas} \Sigma_{1}}\right)
$$

and next the function $p$,

$$
p=(c I+r)^{-1}
$$

where $c$ is a constant dependent on $\frac{1}{\text { meas } \Sigma_{1}}\left(\frac{1}{m}+M\right)$, where $\Sigma_{1}=(0$, $T] \times \Gamma_{1}$.

Step 2. Having constructed the function $p(\cdot)$ in (11.2.23) from the given boundary feedback functions $h_{i}, g_{i}$ (data of the problem) via (11.2.21)-(11.2.23), we next introduce the function $q(\cdot)$ by [L-Ta.1]

$$
q=I-(I+p)^{-1}
$$

so that $q$ is monotone increasing and $q(0)=0$. It is such function $q$ that defines the non-linear ordinary differential equation in the Cauchy problem (11.2.20), whose solution $s(\cdot)$ determines the decay rate of the energy $E(t)$ in (11.2.9) as $t \rightarrow \infty$. 
Remark 11.2.1. (i) Assume, in particular, that the nonlinear functions $g_{i}, h_{i}$ are bounded from below by a linear function; that is, that (reinforcing (11.2.18) valid for $|s|>R$ ):

$$
\left|h_{i}(s)\right| \geq c|s|, \quad\left|g_{i}(s)\right| \geq c|s|, \forall s \in \mathbb{R},
$$

for some $c>0$. Then, it can be shown that the decay rates predicted by Theorem 11.2.2 are exponential. That is, there exist positive constant $C$, $\omega$-possibly depending on $E(0)$ - such that

$$
E(t) \leq C e^{-\omega t}, \quad \forall t \geq T_{0} .
$$

(ii) Assume, instead, that the functions $h_{i}, g_{i}$ have polynomial growth at the origin; that is

$$
\begin{gathered}
h_{i}(s) s \geq a_{i}|s|^{p+1} ; \quad g_{i}(s) s \geq b_{i}|s|^{p+1} \\
\text { for }|s| \leq 1: a_{i}, b_{i} \text { positive constants, } p>1 .
\end{gathered}
$$

Then, the decay rates predicted by Theorem 11.2.2 are algebraic:

$$
E(t) \leq C t^{\frac{2}{1-p}}, \quad p>1,
$$

where $C=C(E(0))=$ a constant depending on $E(0)$.

\subsection{Preliminary results.}

Dissipativity equality. A starting point is, as usual, the dissipativity equality which states that the energy $E(t)$ in (11.2.9) of the entire system is nonincreasing. This is $(0.6)$, as specialized to the present case. This fact alone does not prove, of course, that the energy is decaying, but it is a necessary preliminary step of the stability analysis.

LEMMA 11.3.1. Let $[W, w]$ be a finite energy solution of system (11.1.1), as guaranteed by Theorem 11.2.1. Then, for any $s \leq t$, the following identity holds true for the energy $E(t)$ defined by (11.2.9),

$$
\begin{aligned}
& E(t)+2 \int_{s}^{t} \int_{\Gamma_{1}}\left[g_{1}\left(\left\langle W_{t}, n\right\rangle\right)\left\langle W_{t}, n\right\rangle+g_{2}\left(\left\langle W_{t}, \tau\right\rangle\right)\left\langle W_{t}, \tau\right\rangle\right. \\
& \left.+h_{1}\left(\frac{\partial w_{t}}{\partial n}\right) \frac{\partial w_{t}}{\partial n}+h_{2}\left(\frac{\partial w_{t}}{\partial \tau}\right) \frac{\partial w_{t}}{\partial \tau}\right] d \Gamma_{1} d t=E(s) .
\end{aligned}
$$

Proof. The proof is standard and follows by a classical energy-type argument. We multiply the two equations (11.1.1a-b) by $W_{t}, w_{t}$, respectively, integrate over $\Omega \times(s, t)$ and apply the divergence theorem (as in [Y.3-4]) first to smooth solutions, and then we extend by density to all weak solutions. The main tool of our computations is the following Green's formula $[Y .4,(3.1 .35)]$ in the notation of [Y.4]: 


$$
\begin{aligned}
(\mathcal{A} \eta, \hat{\eta})_{L^{2}(\Omega, \Lambda) \times L^{2}(\Omega)}= & \int_{\Omega}(\mathcal{A} \eta, \hat{\eta}) d x \\
= & \int_{\Omega} B(\eta, \hat{\eta}) d x-\int_{\Gamma}\left\{B_{1}(W, w)\langle\hat{W}, n\rangle\right. \\
& +B_{2}(W, w)\langle\hat{W}, \tau\rangle+\gamma\left[\left(\Delta w+(1-\mu) B_{0} w\right) \frac{\partial \hat{w}}{\partial n}\right. \\
& \left.\left.\left.-\left(\frac{\partial \Delta w}{\partial n}+(1-\mu) B_{4} w\right) \hat{w}\right)\right]\right\} d \Gamma,
\end{aligned}
$$

where $\eta=[W, w], \hat{\eta}=[\hat{W}, \hat{w}]$,

$$
\mathcal{A} \eta \equiv\left[\begin{array}{c}
-\Delta_{\mu} W-(1-\mu) k W-\mathcal{F}(w) \\
\gamma\left[\Delta^{2} w-(1-\mu) \delta(k d w)\right]+\left(H^{2}-2(1-\mu) k\right) w+\mathcal{G}(W)
\end{array}\right],
$$

and where $\Delta_{\mu}$ and $B(\cdot, \cdot)$ are defined below (11.1.1g) and by (11.2.6), while $B_{1}, B_{2}, B_{3}, B_{4}$ are defined by (11.1.2)-(11.1.5). Finally, the coupling $\mathcal{F}(w)$ and $\mathcal{G}(W)$ are first-order operators, defined in [Y.3, (1.33), p. 1733] whose precise structure is not essential in the present paper. However, $\mathcal{F}(w)=0, \mathcal{G}(W)=0$ in the flat case.

In our next step we apply multipliers to (11.1.1). These are the same as those used in the flat non-linear case in [Las.5], except that now they are in differential geometric form. The corresponding calculations in the curved shell case are the Riemannian metric counterpart of those in the flat case, and thus follow the same philosophy as those in [Las.5] where they were used for the full non-linear von Karman system. More precisely, we apply:

(i) the multipliers $\left[D_{V} W, V(w)\right]$, in the usual notation, in order to handle the potential energy $E_{p}(t)$ in (1.2.12), where $V$ is the vector field on $M$ assumed in (H.2);

(ii) the multipliers $[W, w]$, in order to obtain an estimate for the difference between kinetic energy $E_{k}(t)$ and potential energy $E_{p}(t)$.

The actual computations are performed in [Y.3, particularly formulas (2.122), (2.123)] and lead to the following inequality, which is the counterpart, in the curved case, of a special case of the inequality in the flat case given in [Las.5, Lemma 3.2], when specialized to the linear model (11.1.1a-b).

Proposition 11.3.2. Assume (H.1), (H.2). With reference to strong solutions of the original problem (11.1.1a-g) as guaranteed by Theorem 11.2.1, the following inequality holds true for the energy $E(t)$ defined in (11.2.9): for $T>0$ given, there exist constants $C>0, C_{T}>0$, such that

$$
\begin{aligned}
\int_{0}^{T} E(t) d t \leq & C[E(0)+E(T)]+C_{T}\left(B T^{\text {good }}\right) \\
& +C_{T}\left(B T^{\text {bad }}\right)+\operatorname{LOT}(W, w)
\end{aligned}
$$


where:

(i) $\operatorname{LOT}(W, w)$ are lower terms with respect to the energy $E(t)$ in (11.2.9) where $E(t)$ is topologically equivalent to

$$
H^{1}(\Omega, \Lambda) \times L_{2}(\Omega, \Lambda) \times H^{2}(\Omega) \times H^{1}(\Omega)
$$

for $\left[W, W_{t}, w, w_{t}\right]$, see [He.1], [Y.3-4], [L-T.29] for these spaces;

(ii) if $B T=B T^{\mathrm{good}}+B T^{\mathrm{bad}}$ are the boundary terms, divided into 'good' and 'bad' terms, these are defined by

$$
\begin{aligned}
B T^{\mathrm{good}} & =\int_{0}^{T}\left[\left\|W_{t}\right\|_{L^{2}\left(\Gamma_{1}, \Lambda\right)}^{2}+\left\|D w_{t}\right\|_{L^{2}\left(\Gamma_{1}, \Lambda\right)}^{2}\right] d t \\
B T^{\mathrm{bad}} & =\int_{0}^{T}\|D W\|_{L^{2}\left(\Gamma_{1}, T^{2}\right)}^{2} d t+\int_{\Sigma_{1}} B(\zeta, \zeta) d \Sigma_{1},
\end{aligned}
$$

where we recall $\zeta=[W, w]$ and $B(\zeta, \zeta) \equiv a(\Upsilon(\eta), \Upsilon(\eta))+a\left(D^{2} w, D^{2} w\right)$ as in (11.2.6).

Remark 11.3.1. While all boundary terms $B^{\text {good }}$ involving time derivatives in (11.3.6a) will be determined by the dissipation, the boundary integral in the term $B T^{\text {bad }}$ in (11.3.6b) contains traces of the first order for $W$ and of the second order for $w$, see $(11.2 .6),(11.1 .6)$. These traces are not determined either by the energy or by the boundary conditions. In fact, the main challenge to, and contribution by, this paper is to provide an estimate for the traces in the $B T$-term. This will be done by extending microlocal estimates ([L-T.12] for scalar waves, hence [Hn.1] for elastic waves in Lame form, to the present $W$-component which is now in Lame form, and [LT.13] for the plate $w$-component and applying arguments as in [Las.5] for the case of full von Karman model.

11.4. First step of the proof: Local reduction to a Euclidean (flat) coordinate system. Throughout this section Assumptions (H.1) and Assumption (H.2) are in force. The following estimate is critical for the proof of Theorem 11.2.2.

THEOREM 11.4.1. Let $0<\alpha<T / 2$. Then, the following trace estimate holds true for any regular solution of problem (11.1.1), as guaranteed by Theorem 11.2.1: there exists a constant $C_{\alpha T}>0$ such that

$$
\begin{aligned}
B T^{\mathrm{bad}}[\alpha, T-\alpha] \equiv & \int_{\alpha}^{T-\alpha} \int_{\Gamma_{1}}\left[B(\zeta, \zeta)+|D W|_{T_{x}^{2}}^{2}\right] d x d t \\
\leq & C_{\alpha T} \int_{\Sigma_{1}}\left[\left|D w_{t}\right|_{T_{x}}^{2}+\left|W_{t}\right|_{T_{x}}^{2}+\left|g_{1}\left(\left\langle W_{t}, n\right\rangle\right)\right|^{2}\right. \\
& +\left|g_{2}\left(\left\langle W_{t}, \tau\right\rangle\right)\right|^{2}+\left|h_{1}\left(\frac{\partial w_{t}}{\partial n}\right)\right|^{2} \\
& \left.+\left|h_{2}\left(\frac{\partial w_{t}}{\partial \tau}\right)\right|^{2}\right] d \Sigma_{1}+\operatorname{LOT}(W, w) .
\end{aligned}
$$


where the lower order terms LOT are below energy level and satisfy

$$
\begin{aligned}
\operatorname{LOT}(W, w) \leq & C_{\epsilon} \sup _{t \in[0, T]}\left[|W(t)|_{1-\epsilon, \Omega}+\left|W_{t}(t)\right|_{-\epsilon, \Omega}\right. \\
& \left.+|w(t)|_{2-\epsilon, \Omega}+\left|w_{t}(t)\right|_{1-\epsilon, \Omega}\right] .
\end{aligned}
$$

for any $\epsilon>0$, see notation adopted in [ ] in 1-forms $W, W_{t}$ or functions $w, w_{t}$.

Remark 11.4.1. The traces $B(\zeta, \zeta), D W$ in (11.4.1) are not bounded by the energy.

Proof. By recalling the definition of $B(\zeta, \zeta)$ in (11.2.6) we see that it suffices to prove the following estimate for regular solutions of problem (11.1.1): there exists a constant $C_{\alpha T}>0$, such that

$$
\begin{aligned}
& \int_{\alpha}^{T-\alpha} \int_{\Gamma_{1}}\left[\left|D^{2} w\right|_{T_{x}^{2}}+|D W|_{T_{x}^{2}}^{2}\right] d x d t \\
& \leq C_{\alpha T} \int_{\Sigma_{1}}\left[\left|D w_{t}\right|_{T_{x}}^{2}+\left|W_{t}\right|_{T_{x}}^{2}+\left|g_{1}\left(\left\langle W_{t}, n\right\rangle\right)\right|^{2}\right. \\
&+\left|g_{2}\left(\left\langle W_{t}, \tau\right\rangle\right)\right|^{2}+\left|h_{1}\left(\frac{\partial w_{t}}{\partial n}\right)\right|^{2} \\
&\left.+\left|h_{2}\left(\frac{\partial w_{t}}{\partial \tau}\right)\right|^{2}\right] d \Sigma_{1}+\operatorname{LOT}(W, w)
\end{aligned}
$$

Orientation. The proof of estimate (11.4.3) requires the insertion of microlocal estimates. To carry this out, we shall apply a basic strategy similar to that already employed in [Las.5] in the flat, nonlinear case of the full von Karman system. Indeed, the proof of estimate (11.4.3) will comprise three main steps. In Step 1, one introduces a coordinate cover of a boundary layer of $\bar{\Omega}$ and a subordinate partition of unity, and reduce our task to prove estimate (11.4.3) for just one coordinate system. Next, in Step 2 , see Subsection 11.5, one provides a sharp trace estimate for the linear model of dynamic elasticity, to be used for the in-plane $W$-components of the displacement vector $\zeta$. Finally, in Step 3, see Subsection 11.6, one provides a sharp trace estimate for the linear Kirchoff plate model, to be used for the normal $w$-component of the displacement $\zeta$.

Steps 2 and 3 are critical for the proof of the stabilizability estimates without assuming geometric conditions on the controlled portion $\Gamma_{1}$ of the boundary, as done in wave-plate literature [Lag.2-3] and without considering additional tangential components of the horizontal displacement $W$ in the structure of the stabilizing feedback as done in [P-T.1] in the flat case.

11.5. Trace regularity for elastic waves (W-component). Orientation. This subsection and the next provide sharp trace regularity 
results which are critical for the proof of stability estimates without assuming geometric conditions on $\Gamma_{1}$ and without considering artificial tangential components of the in-plane displacement $W$ in the structure of the stabilizing feedback (as was done in [P-T.1] in the study of the von Karman problem) which are not in $L_{2}$. These estimates are based on the corresponding trace estimates valid for: (i) linear model of dynamic elasticity and (ii) linear Kirchhoff model. They are obtained by methods of microlocal analysis.

As to (i), we need to extend to the present non-Lame elastic $W$ component the analysis begun in [L-T.12] for second-order hyperbolic equations, which was then the basis for the analysis in [Hn.1] of Lame-type elastic systems. We cannot merely quote [Hn.1] as our $W$-system is not of Lame-type. As to (ii) instead, we shall invoke [L-T.13] for the sharp trace regularity of second-order derivatives for plates. The sharp trace regularity of first-order traces for $W$ - to be given in Proposition 11.5.1 below-are critical for solving the stabilization problem in the first place. Instead, the sharp trace regularity of second-order traces for $w$ - to be given in Proposition 11.6.1 - merely avoid unnecessary and restrictive geometrical conditions on the controlled portion of the boundary $\Gamma_{1}$. The main idea is to obtain the estimates for the tangential derivatives on the boundary in terms of the velocity traces and lower order terms: see Proposition 11.5.1 for $W$ and Proposition 11.6.1 for $w$.

The constant $C$ is a generic constant, different in various occurrences.

Proposition 11.5.1. Let $W, w$ be a finite energy solution corresponding to the system (11.1.1) as guaranteed by Theorem 11.2.1(a). Then, for any $0<\epsilon<1 / 4$ and $0<\alpha<T / 2$, there exist constants $C>0$ such that the following trace regularity takes place:

$$
\begin{aligned}
\int_{\alpha}^{T-\alpha} \int_{\Gamma_{1}}|D W|_{T_{x}^{2}}^{2} d \Sigma_{1 \alpha} \leq & C_{\alpha \epsilon T} \int_{\Sigma_{1}}\left|W_{t}\right|^{2}+\left|g_{1}\left(\left\langle W_{t}, n\right\rangle\right)\right|^{2} \\
& +\left|g_{2}\left(\left\langle W_{t}, \tau\right\rangle\right)\right|^{2} d \Sigma_{1} \\
& +C_{\alpha T} \int_{0}^{T}\left[|w|_{2-\epsilon, \Omega}^{2}+|W|_{1-\epsilon, \Omega}^{2}\right] d t .
\end{aligned}
$$

Remark 11.5.1. Proposition 11.5.1 is a counterpart of [Las.5, Lemma $2.2]$, proved for the "flat" but nonlinear case. Notice that the regularity of the trace of $D W$, claimed by Proposition 11.5.1 (see also Proposition 11.6.1 for the Kirchhoff plate below) does not follow from the standard interior regularity of finite energy solutions via trace theory. These are independent regularity results which rely heavily on microlocal arguments applied to both: the dynamic system of elasticity and the dynamic Kirchhoff plate.

Remark 11.5.2: Orientation. Proposition 11.5.1 is established by introducing a coordinate cover of the boundary layer of $\bar{\Omega}$ and a subordinate partition of unity, and thus by proving a corresponding estimate in the 
Euclidean setting just for one coordinate system. This latter estimate for the trace of the elastic space in the Euclidean case cannot be just quoted from available literature, since the resulting system is not Lame-type, as in [Hn.1]. Indeed, two additional technical lemmas need to be established for this more general elastic wave problem [L-T.29], which push further to the present level of generality arguments in the literature [L-T.12], [L-T.13].

11.6. Trace regularity for normal component $\boldsymbol{w}$. Our next result deals with the improved trace regularity for the normal displacement $w$.

Proposition 11.6.1. Let $W, w$ be a finite energy solution to problem (11.1.1) as guaranteed by Theorem 11.2.1. Then, there is a constant $C_{\alpha T \epsilon}>0$ such that

$$
\begin{aligned}
\int_{\alpha}^{T-\alpha} & \int_{\Gamma_{1}}\left|D^{2} w\right|_{T_{x}^{2}}^{2} d \Sigma_{1 \alpha} \\
\leq & C\left\{\int_{\Sigma_{1}}\left[\left|D w_{t}\right|^{2}+\left|h_{1}\left(\frac{\partial w_{t}}{\partial n}\right)\right|^{2}+\left|h_{2}\left(\frac{\partial w_{t}}{\partial \tau}\right)\right|^{2}\right] d \Sigma_{1}\right. \\
& \left.+\int_{0}^{T}\left[|w(t)|_{2-\epsilon, \Omega}^{2}+\left|w_{t}(t)\right|_{1-\epsilon, \Omega}^{2}+|W(t)|_{1-\epsilon, \Omega}^{2}\right] d t\right\} .
\end{aligned}
$$

Remark 11.6.1. Again Proposition 11.6.1 is established (as Proposition 11.5.1) by introducing a coordinate cover of the boundary layer of $\bar{\Omega}$ and a subordinate partition of unity, and thus by proving a corresponding estimate in the Euclidean setting just for one coordinate system. This latter estimate for the corresponding Kirchhoff plate problem is then lifted from [L-T.13, Theorem 2.1, p. 279], except that we are giving it a sharper form. This is explained in [L-T.29, Remark 3.3.1].

11.7. Stabilizability estimate and completion of the proof of Theorem 11.2.2. The remaining part of the proof of Theorem 11.2.2 is contained (as a strict subset) in the arguments presented in [L-Ta.1]. For the reader's convenience, we shall outline the remaining steps.

Step 1. We proceed as in [L-T.12, Section 7.3], [Las-Ta.1]. We return to estimate (11.3.4) of Proposition 11.3.2, this time over the interval $[\alpha, T-$ $\alpha]$ rather than over $[0, T]$. On the left-hand side, we obtain $(T-2 \alpha) E(T) \leq$ $\int_{\alpha}^{T-\alpha} E(t) d t$ by the dissipativity property in (11.3.1). On the right-hand side, we use: (i) estimate (11.4.1) of Theorem 11.4.1 for the boundary terms $B T^{\text {bad }}[\alpha, T-\alpha]$ over $[\alpha, T-\alpha]$; (ii) identity (11.3.1) again with $s=0, t=T$, to express $E(0)$ in terms of $E(T)$ plus boundary terms, where we use: $2 g_{i}(s) s \leq\left|g_{i}(s)\right|^{2}+s^{2} ; 2 h_{i}(s) s \leq\left|h_{i}(s)\right|^{2}+s^{2}$ for these boundary terms, as well as the relevant definitions to estimate them by the norms of $D w_{t}$ and $W_{t}$. This way, using again (11.3.4), we readily obtain the counterpart of [L-Ta.1, Lemma 3.3].

Proposition 11.7.1. With reference to the energy in (11.2.9), the strong solutions of the original dynamics (11.1.1), guaranteed by Theorem 
11.2.1, satisfy the following estimate for all $T>0$ sufficiently large, there is a constant $C_{T}>0$ such that

$$
\begin{aligned}
E(T)+\int_{0}^{T} E(t) d t \leq & C_{T} \int_{\Sigma_{1}}\left\{\left\|W_{t}\right\|_{L^{2}(\Gamma, \Lambda)}^{2}+\left\|D w_{t}\right\|_{L^{2}(\Gamma, \Lambda)}^{2}\right. \\
& +\left|g_{1}\left(\left\langle W_{t}, n\right\rangle\right)\right|^{2}\left|g_{2}\left(\left\langle W_{t}, \tau\right\rangle\right)\right|^{2}+\left|h_{1}\left(\frac{\partial w_{t}}{\partial n}\right)\right|^{2} \\
& \left.+\left|h_{2}\left(\frac{\partial w_{t}}{\partial \tau}\right)\right|^{2}\right\} d \Sigma_{1}+\operatorname{LOT}(W, w)
\end{aligned}
$$

where, as before, $\operatorname{LOT}(W, w)$ are lower terms (with respect to the energy) as defined in (11.4.2).

Step 2. Lower-order terms are absorbed, as usual, by a compactness/uniqueness argument. This requires an appropriate unique continuation result (from the boundary). Since the coefficients are time-independent, it is far convenient to require a uniqueness result for the corresponding static problem. The latter is established in [Y.4, Proposition 2.3], by reducing the Cauchy problem to a system of three equations of the fourth-order with the same principal part $\Delta^{2}$, where $\Delta$ is the Laplacian on the manifold $M$. For this latter problem, the result in [S.1] is then invoked to obtain uniqueness. (One could also use results of [I. ]). Thus, a by-now standard compactness/uniqueness argument (see [L-Ta.1, Lemma 4.1] leads to the absorption of lower-order terms. We thus obtain

LEMMA 11.7.2. With reference to the $\operatorname{LOT}(W, w)$ in estimate (11.7.1) for the strong solutions of problem (11.1.1), there exists $T>0$ large enough, so that:

$$
\begin{aligned}
\operatorname{LOT}(W, w) \equiv & \int_{0}^{T}\left[|W(t)|_{1-\epsilon, \Omega}^{2}+|w(t)|_{2-\epsilon, \Omega}^{2}\right] d t \\
\leq & C_{T}\left[\int _ { \Sigma _ { 1 } } \left[\left|W_{t}\right|^{2}+\left|D w_{t}\right|^{2}+\left|g_{1}\left(\left\langle W_{t}, n\right\rangle\right)\right|^{2}\right.\right. \\
& \left.+\left|g_{2}\left(\left\langle W_{t}, \tau\right\rangle\right)\right|^{2}+\left|h_{1}\left(\frac{\partial w_{t}}{\partial n}\right)\right|^{2}+\left|h_{2}\left(\frac{\partial w_{t}}{\partial \tau}\right)\right|^{2}\right] d \Sigma_{1}
\end{aligned}
$$

Step 3. By combining the results of Proposition 11.7.1 and Lemma 11.7.2, we obtain the final stabilization estimate.

Proposition 11.7.3. Let $[W, w]$ be a regular solution to the original system (11.1.1), as guaranteed by Theorem 11.2.1. Then, there exists a constant $T_{0}>0$ such that for any $T>T_{0}$, there is a constant $C_{T}>0$ such that the following estimate holds true:

$$
\begin{aligned}
E(0)+E(T)+\int_{0}^{T} E(t) d t \leq & C_{T} \int_{\Sigma_{1}}\left[\left|W_{t}\right|^{2}+\left|D w_{t}\right|^{2}\right. \\
& +\left|g_{1}\left(\left\langle W_{t}, n\right\rangle\right)\right|^{2}+\left|g_{2}\left(\left\langle W_{t}, \tau\right\rangle\right)\right|^{2}
\end{aligned}
$$




$$
\left.+\left|h_{1}\left(\frac{\partial w_{t}}{\partial n}\right)\right|^{2}+\left|h_{2}\left(\frac{\partial w_{t}}{\partial \tau}\right)\right|^{2}\right] d \Sigma_{1} .
$$

Step 4. Our next step is to express the boundary terms in terms of the feedbacks in (11.1.1d-f). To accomplish this, we shall use the growth conditions (11.2.18) imposed on the non-linear dissipation terms $g_{i}, h_{i}$ at infinity, together with the consequent properties (11.2.21) of the "comparison" functions $\tilde{g}_{i}, \tilde{h}_{i}$ which contain information on the growth at the origin. It is only at this point that we use the growth conditions (11.2.18) imposed on the non-linear functions $g_{i}, h_{i}$ given in assumption $($ H.3 $)=(11.2 .18)$, together with the construction of the functions $\tilde{g}_{i}, \tilde{h}_{i}$ which capture the behavior of the non-linearity at the origin.

By splitting the integration on the boundary between "low" and "high" frequencies, and using Jensen's inequality in the same manner as it is used on [L-Ta.1, p. 1400-1401], we arrive at the following conclusion.

Proposition 11.7.4. Consider regular solutions of problem (11.1.1), as guaranteed by Theorem 11.2.1. Let $E(t)$ be defined in (11.2.9). Then: there exists $T>0$ such that

$$
p(E(T))+E(T) \leq E(0),
$$

where the monotone function $p$ is defined constructively in (11.2.23).

Step 5. The final conclusion of Theorem 11.2.2 now follows from (11.7.4) and [L-Ta.1, Lemma 3], which is based on a comparison theorem.

\section{REFERENCES}

[B-L-R.1] C. BARdos, G. Lebeau, AND J. RAUCh, Sharp sufficient conditions for the observation, control, and stabilization of waves from the boundary, SIAM J. Control Optim. 30 (1992), 1024-1065.

[B-L-R-H-Z.1] C. Bardos, G. Lebeau, J. Rauch, L. Halperin, and E. Zuazua, Stabilisation de l'equation des ondes au moyen d'un feedback portant sur la condition aux limites de Dirichlet, Asymptotic Analysis 4 (1991), 285-291.

[B-B.1] M. Bernadou And J.M. Boisserie, The Finite Element Method in Thin Shell Theory, Progress in Scientific Computing 1, Birkhäuser, Boston, 1982.

[B-O.1] M. Bernadou and J.T. Oden, An existence theorem for a class of nonlinear shallow shell problems, J. Math. Pures et Appl. 60 (1981), 258-298.

[B-G-L.1] S. Betelu, R. Gulliver, and W. Littman, Curvature flow methods in boundary control of partial differential equations, Appl. Math. \&5 Optimiz. 46 (Sept./Dec. 2002), 167-178. Special issue dedicated to J.L. Lions.

[B-C] R. Bishop And R. CRittenden, Geometry of Manifolds, Academic Press, New York 1964.

[B-Z] Yu. D. Burago And V.A. Zalgaller, Geometric Inequalities, Springer-Verlag, Berlin 1988.

[B.1] N. BurQ, Controlabilite exacte des ondes dans des ouverts peu reguliers, Asymptotic Analysis 14 (1997), 157-191. 
[Ch.1]

$[\mathrm{E}-\mathrm{I}-\mathrm{N}-\mathrm{T}]$
I. Chavel, Riemannian Geometry, a Modern Introduction, Cambridge University Press, Cambridge 1993.

J. Cheeger and D. Gromoll, On the structure of complete manifolds of nonnegative curvature. Annals of Math. 96 (1972), 413-443.

K.-S. Chou And X.-P. Zhu, The Curve Shortening Problem, Chapman and Hall, 2001.

J. Choe And R. Gulliver, Isoperimetric inequalities on minimal submanifolds of space forms, Manuscripta Math. 77 (1992), 169-189.

R. Courant And D. Hilbert, Methods of Mathematical Physics, Vol. 2, Wiley Interscience, New York, 1962.

M.P. Do CARmo, Riemannian Geometry, Birkhäuser, Boston 1992.

G.F.D. DufF, Partial Differential Equations, University of Toronto Press, 1956.

M. ElLER, Uniqueness of continuous theorems, in Direct and Inverse Problems of Mathematical Physics, Kluwer Academic, Dordrecht/Norwell, MA, 1999.

M. Eller, Carleman estimates with a second large parameter, JMAA 249 (2000), 491-514.

M. Eller, V. Isakov, G. Nakamura, and D. Tataru, Uniqueness and stability in the Cauchy problem for Maxwell's and the elastic system, in Nonlinear Partial Differential Equations, Vol. 16, Chapman and Hall, London/New York, 2000.

M. Eller, I. Lasiecka, AND R. Triggiani, Simultaneous exact/approximate boundary controllability of thermo-elastic plates with variable transmission coefficients, Marcel Dekker, Lecture Notes Pure Appl. Math., J. Cagnol, M. Polis, and J.P. Zolesio (2001), 109-230.

M. Eller, I. Lasiecka, AND R. Triggiani, Simultaneous exact/approximate boundary controllability of thermoelastic plates with variable thermal coefficient and moment controls, J. Math. Anal. Appl. 251 (2000), 452-478.

M. Eller, I. Lasiecka, and R. Triggiani, Unique continuation for over-determined Kirchoff plate equations and related thermoelastic systems, J. Inv. Ill-Posed Prob. 9(2) (2001), 103-148.

M. Eller, I. Lasiecka, and R. Triggiani, Simultaneous exact/approximate controllability of thermoelastic plates with variable thermal coefficient and clamped controls, Discrete Contin. Dynam. Systems $\mathbf{7}(2)$ (2001), 283-301.

A.V. Fursikov and O.Yu. Imanuvilov, Controllability of Evolution Equations, Lecture Notes, Vol. 34, pp. 151-742, Research Institute of Mathematics, Seoul National University, Seoul, Korea.

M. Galbraith, Geometric optics and convex functions in the boundary control of the wave equation, Proceedings of 3rd ISAAC Conference, 2001, World Scientific, to appear.

M.A. Grayson, Shortening embedded curves, Annals of Math. 129 (1989), 71-111.

R.E. Greene And H. Wu, $C^{\infty}$ convex functions and manifolds of positive curvature, Acta Math. 137 (1976), 209-245.

P. Grisvard, Elliptic Problems in Nonsmooth Domains, Monograph \& Studies in Mathematics, Pitman, 1995.

R. Gulliver, On the variety of manifolds without conjugate points, Trans. Amer. Math. Soc. 210 (1975), 185-201.

R. Gulliver and W. Littman, Chord uniqueness and controllability: The view from the boundary, Contemporary Mathematics, AMS 268 (2000), 145-176. 
[G-L.2] R. Gulliver and W. Littman, The use of geometric tools in the boundary control of partial differential equations, Proceedings of 3rd International ISAAC Contress, Berlin, August 2001.

[He.1] E. Hebey, Sobolev Spaces on Riemannian Manifolds, Lecture Notes in Mathematics, Springer, 1996.

[Ho.1] L.F. Ho, Observabilité frontière de l'equation des ondes, C. R. Acad. Sci. Paris, Ser. I Math. 202 (1986), 443-446.

[Hor.1] L. Hörmander, Linear Partial Differential Operators, Springer Verlag, 1963.

[Hor.2] L. Hörmander, The Analysis of Linear Partial Differential Operators, Vol. III, Springer Verlag, Berlin/New York, 1985.

[Hor.3] L. Hörmander, On the characteristic Cauchy problem, Ann. Math. 88 (1968),

[Hor.4] L. Hörmander, On the uniqueness of the Cauchy problem, II, Math. Scand. 7 (1959), 177-190.

[Hor.5] L. HöRMANDER, On the uniqueness of the Cauchy problem under partial analyticity assumptions, in Geometrical Optics and Related Topics, F. Colombini and N. Lerner (eds.), Birkhäuser, 1997.

[Hn.1] M.A. HoRN, Implications of sharp trace regularity results on boundary stabilization of the system of linear elasticity, J. Math. Anal. Appl. 223 (1998), 126-150.

[Hn.2] M.A. HoRn, Sharp trace regularity of the traces to solutions of dynamic elasticity, J. Math. Systems, Estimation and Control 8 (1998), $217-229$.

[Koi] W.T. KoITeR, On the foundations of the linear theory of thin elastic walls, Proc. Kon. Ned. Akad. Wetensch. B73 (1970), 169-195.

[I.1] V. IsAKOV, A nonhyperbolic Cauchy problem for $\square_{b} \square_{c}$ and its applications to elasticity theory, Comm. PDE's XXXIX (1986), 747-767.

V. Isakov, Inverse Problems for Partial Differential Equations, Springer Verlag, 1998.

V. IsAKOV, Uniqueness of the continuation across a time-like hyperplane and related inverse problems for hyperbolic equations, Comm. Partial Diff. Eqn. 14 (1989), 465-478.

V. Isakov, Inverse Source Problems, AMS, Providence, RI, 1990.

V. ISAKOV, On the uniqueness of the continuation for a thermoelastic system, preprint, 1998.

V. IsAKOV, On uniqueness on a lateral Cauchy problem with multiple characteristics, J. Diff. Eqns. 134 (1997), 134-147.

V. IsAKov AND M. YAmamoto, Carleman estimate with Neumann boundary condition and its application to the observability inequality and inverse hyperbolic problems, Contemporary Mathematics, AMS 268 (2000).

V. KOMORNIK, Exact controllability and stabilization: the multiplier method, Research in Applied Mathematics, 1994, Masson/John Wiley.

[K-K.1] M. KazAmi And M.V. Klibanov, Stability estimates for ill-posed Cauchy problems involving hyperbolic equations and inequalities, Applicable Analysis 50 (1993), 93-102.

[Lag.1] J. LAGNESE, Decay of solutions of the wave equation in bounded domains, JDE 50 (1983), 163-182.

[Lag.2] J. LAGNESE, Uniform asymptotic energy estimates for solutions of the equations of dynamic plane elasticity with nonlinear dissipation at the boundary, Nonlinear Anal. 16 (1991), 35-54.

[Lag.3] J. Lagnese, Boundary Stabilization of Thin Plates, SIAM, Philadelphia, 1989. 
J. Lagnese And J.L. Lions, Modelling, Analysis and Control of Thin Plates, Masson, 1988.

[Las.1] I. LASIECKA, Controllability of a viscoelastic Kirchoff plate, Int. Series Num. Math. 91 (1989), 237-247, Birkhäuser Verlag, Basel.

I. LASIECKA, Mathematical control theory in structural acoustic problems, Mathematical Models and Methods in Applied Sciences $\mathbf{8}$ (1998), 1119-1153.

[Las.3] I. LASIECKA, Boundary stabilization of a 3-dimensional structural acoustic model, J. Math. Pure Appl. 78 (1999), 203-232.

[Las.4] I. LASIECKA, Intermediate solutions to full von Kármán systems of dynamic nonlinear elasticity, Appl. Anal. 68 (1998).

[Las.5]

[Las.6]

I. LASIECKA, Uniform stabilizability of a full von Kármán system with nonlinear boundary feedback, SIAM J. Control 36(4) (1998), $1376-1422$.

[Las.7]

I. LASIECKA, Uniform decay rates for full von Kármán system of dynamic elasticity with free boundary conditions and partial boundary dissipation, Comm. PDE 24 (1999), 1801-1849. CMBS Lecture Notes (2001), SIAM.

[L-M.1] I. LASIECKA AND R. MARCHAND, Uniform decay for solutions to nonlinear shells with nonlinear dissipation, Nonlinear Analysis 30 (1997), $5409-5418$.

[Las-Hor.1] I. LASIECKA AND M.A. HoRn, Asymptotic behavior with respect to thickness of boundary stabilizing feedback for the Kirchoff plate, J. Diff. Eqns. 114(2) (1994), 396-433.

[L-O.1] I. LASIECKA AND J. ONG, Global solvability and uniform decays of solutions to quasilinear equation with nonlinear boundary dissipation, Comm. PDE 24 (11 \& 12) (1999), 2069-2107.

[L-Ta.1] I. LASIECKA AND D. TATARU, Uniform boundary stabilization of semilinear wave equations with nonlinear boundary damping, Diff. Int. Eqns. 6 (1993), 507-533.

[L-T.1] I. LASIECKA AND R. TRIGGiani, Uniform exponential energy decay of wave equations in a bounded region with $L_{2}\left(0, \infty ; L_{2}(\Gamma)\right)$-feedback control in the Dirichlet boundary conditions, J. Diff. Eqns. 66 (1987), 340-390.

[L-T.2] I. Lasiecka And R. Triggiani, Exact controllability of the EulerBernoulli equation with $L_{2}(\Sigma)$-control only in the Dirichlet B.C., Atti Acad. Naz. Lincei Rend. Cl. Sc. Fis. Math. Nat. Vol. LXXXII, No. 1 (1988), Rome.

[L-T.3] I. LASiECKa And R. Triggiani, Exact controllability of the wave equation with Neumann boundary control, Appl. Math. Optimiz. 19 (1989), 243-290.

[L-T.4] I. LASIECKA AND R. TRIGgiani, Exact controllability of the EulerBernoulli equations with controls in the Dirichlet and Neumann B.C.: A nonconservative case, SIAM J. Control Optim. 27 (1989), 330-373.

[L-T.5] I. Lasiecka And R. TRIgGiani, Exact controllability of the EulerBernoulli equation with boundary controls for displacement and moment, JMAA 146 (1990), 1-33.

[L-T.6] I. LASIECKA AND R. TRIGGiani, Sharp regularity for mixed second-order hyperbolic equations of Neumann type, Part I: The $L_{2}$-boundary case, Annal. di Matemat. Pura e Appl. (IV) 157 (1990), 285-367.

[L-T.7] I. Lasiecka AND R. Triggiani, Exact controllability of the semilinear abstract system with application to waves and plates, Appl. Math. Optimiz. 23 (1991), 109-154. 
[L-T.8] I. Lasiecka And R. TRiggiani, Optimal regularity, exact controllability and uniform stabilization of the Schrödinger equation, Diff. Int. Eqns. 5 (1991), 521-535.

[L-T.9] I. LASIECKA AND R. TRIGGIANI, Exact controllability and uniform stabilization of Kirchoff plates with boundary controls only in $\left.\Delta w\right|_{\Sigma}$, J. Diff. Eqns. 93 (1991), 62-101.

[L-T.10] I. LASIECKA AND R. TRIGGIANI, Regularity theory of hyperbolic equations with non-homogeneous Neumann boundary conditions, Part II: General boundary data, J. Diff. Eqns. 93 (1991), 112-164.

[L-T.11] I. Lasiecka And R. Triggiani, Differential and Algebraic Riccati Equations with Applications to Boundary/Point Control Problems: Continuous Theory and Approximation Theory, Springer Verlag LNICS, Vol. 164 (1991).

[L-T.12] I. LASIECKA AND R. TRIGGIANI, Uniform stabilization of the wave equation with Dirichlet or Neumann feedback control without geometric conditions, Appl. Math. Optim. 25 (1992), 189-224.

[L-T.13] I. LASIECKA AND R. TRIGGiani, Sharp trace estimates of solutions to Kirchhoff and Euler-Bernoulli equations, Appl. Math. Optimiz. 28 (1993), 277-306.

[L-T.14] I. LasiecKa and R. Triggiani, A-Priori Observability Inequalities, Chapter 1, Lecture Notes, University of Virginia, May 1995.

[L-T.15] I. LasieCKa and R. Triggiani, Abstract models and semigroup wellposedness of spherical shells with boundary dissipation, invited paper, Dynam. Sys. Appl. 4 (1995), 453-471, World Scientific Series in Applicable Analysis.

[L-T.16] I. Lasiecka And R. Triggiani, Carleman estimates and uniqueness for the system of strongly coupled PDEs of spherical shells, special volume of Zeitschrift für Angewandte Mathematik and Mechanik, ZAMM, Akademie Verlag, Berlin, ICIAM 1995, 76, supp. 4 (1996), $277-280$.

[L-T.17] I. Lasiecka and R. Triggiani, Carleman Estimates and Exact Boundary Controllability for a System of Coupled, Non-Conservative Second-Order Hyperbolic Equations, Lecture Notes in Pure and Applied Mathematics, Vol. 188, pp. 215-243, Marcel Dekker, New York, 1997, J.P. Zolesio and G. Da Prato (eds.).

[L-T.18] I. LASIECKA AND R. TRIGGiani, Two direct proofs of analyticity of the s.c. semigroup arising in abstract thermo-elastic equations, $A d$ vances in Diff. Eqns. 3 (1998), 387-416.

[L-T.19] I. Lasiecka And R. Triggiani, Analyticity of thermo-elastic semigroups with coupled hinged/Neumann B.C., Abstract Appl. Anal. 3(1-2) (1998), 153-169.

[L-T.20] I. Lasiecka and R. Triggiani, Analyticity of thermo-elastic semigroups with free B.C., Annali Scuola Normale Superiore, Pisa, cl. sci. (4), XXVII (1998).

[L-T.21] I. LASIECKA AND R. TRIGGIANI, Exact null controllability of structurally damped and thermo-elastic parabolic models, Memoria, Academia dei Lincei Rome (Italy), Sezione Matematica 9 (1998), 943-969.

[L-T.22] I. LASIECKA AND R. TRIGGiani, Analyticity, and lack thereof, of thermo-elastic semigroups, European Soc. Appl. Math. (ESIAM) 4 (1999), 199-222.

[L-T.23] I. LASIECKA AND R. Triggiani, sharp trace result on a thermo-elastic plate equation with coupled hinged/Neumann boundary conditions, Discrete Cont. Dynam. Sys. 5 (1999), 585-599.

[L-T.24] I. Lasiecka and R. Triggiani, Control Theory for Partial Differential Equations, Vol. 1 (680 pp.) and Vol. II (446 pp.), Cambridge University Press, 2000. 
[L-T.25] I. Lasiecka and R. Triggiani, Feedback Noise Control in an Acoustic Chamber: Mathematical Theory, Gordon and Breach Scientific Publishers, 2000, 89-111.

[L-T.26] I. LASIECKA AND R. TRIGGiani, Exact boundary controllability of a first order non-linear hyperbolic equation with non-local integral term arising in epidemic modeling, Direct and Inverse Problems of Matheamtical Physics R.P. Gilbert, J. Kajiwara, U. Xu (Editors), Kluwer (2000), 363-398.

[L-T.27] I. Lasiecka AND R. Triggiani, Structural decomposition of thermoelastic semigroups with rotational forces, Semigroup Forum $\mathbf{6 0}$ (2000), 1-61.

[L-T.28] I. Lasiecka and R. Triggiani, A sharp trace result of Kirchof and thermo-elastic plate equations with free boundary conditions, Rocky Mtn. J. Math. 30(3) (Fall 2000), 981-1024.

[L-T.29] I. LASIECKA AND R. TRIGGiani, Uniform stabilization of a shallow shell model with nonlinear boundary feedbacks, J. Math. Anal. Appl. 269 (2002), 642-688.

[L-T.30] I. LASIECKA AND R. TRIGGiani, $L_{2}(\Sigma)$-regularity of the boundary $\rightarrow$ boundary operator $B^{*} L$ for hyperbolic and Petrowski PDEs, submitted.

[L-L-T.1] I. Lasiecka, J.L. Lions, And R. TRIgGiani, Nonhomogeneous boundary value problems for second-order hyperbolic operators, J. Math. Pures Appl. 65 (1981), 149-192.

[L-T-V.1] I. Lasiecka, R. Triggiani, and W. Valente, Uniform stabilization of spherical shells by boundary dissipation, Advances Diff. Eqns. $1(4)$ (1996), 635-674.

[L-V.1] I. LASIECKA AND W. VALENTE, Uniform stabilization of a nonlinear shallow and tin spherical cap, JMAA 22 (1996), 951-994.

[L-T-Y.1] I. LASiecka, R. Triggiani, And P-F. YaO, Exact controllability for second-order hyperbolic equations with variable coefficientprincipal part and first-order terms, Nonlinear Anal. 30 (1997), $111-122$.

[L-T-Y.2] I. Lasiecka, R. Triggiani, and P-F. YaO, Inverse/observability estimates for second-order hyperbolic equations with variable coefficients, J. Math. Anal. Appl. 235 (1999), 13-57.

[L-T-Y.3] I. LASIECKA, R. TRigGiani, AND P-F. YAO, An observability estimate in $L_{2} \times H^{-1}$ for second-order hyperbolic equations with variable coefficients, Control of Distributed Parameter and Stochastic Systems, S. Chen, X. Li, J. Yong, X. Zhou (eds.), Kluwer Academic Publishers, Dordrect, 1999.

[L-T-Y.4] I. Lasiecka, R. TRiggiani, and P-F. Yao, Carleman estimates for a plate equation on a Riemannian manifold with energy level terms, Analysis and Applications, Chapter 15, 199-236 ISAAC, 2001 Kluwer, to appear in 2003.

[L-T-Z.1] I. LAsieCKA, R. Triggiani, And X. Zhang, Nonconservative wave equations with unobserved Neumann B.C.: Global uniqueness and observability in one shot, Contemporary Mathematics, AMS 268 (2000), 227-326.

[L-T-Z.2] I. Lasiecka, R. Triggiani, And X. Zhang, Nonconservative Schrödinger equations with unobserved Neumann B.C.: Global uniqueness and observability in one shot, Spring 2002. Announcement, under the same title, to appear in the Proceedings of the Conference, "Analysis and Optimization of Differential Systems," held at Ovidius University, Constanta, Romania, Sept. 10-15, 2002.

[L-R-S.1] M.M. Lavrent'Ev, V.G. Romanov, and S.P. Shishat-Skit, Ill-Posed Problems of Mathematical Physics and Analysis, English transla- 
[Le.1]

[Le.2]

[Le.3]

[Lit.3]

[Lit-Li.1]

[Lit-Ta.1] tion. (The original: Nauka Moscow, 1980), American Mathematical Society, Providence, RI, 1986.

G. Lebeau, Contrôl analytique, I, Estimations á priori, Duke Math. J. 68 (1992), 1-30.

G. Lebeau, Controle de l'equation de Schrödinger, J. Math. Pures Appl. 71 (1992), 267-291.

G. Lebeau, Controle des ondes amorties, Prepublications de Université de Paris-Sud 19 (1994).

J.L. Lions, Controllabilite Exacte, Stabilization et Perturbation des Systemes Distribues, Vol. 1, Masson, Paris, 1988.

W. LitTman, Near-optimal-time boundary controllability for a class of hyperbolic equations, Control Problems for Systems Described by Partial Differential Equations and Applications (Gainesville, FL, 1986), 307-312, Lecture Notes in Control and Inform. Sci. 97, Springer, Berlin-New York, 1987.

W. LitTman, Remarks on global uniqueness theorems for partial differential equations, Contemporary Mathematics, AMS 268 (2000).

W. LitTMAN, Boundary controllability for polyhedral domains, LNCIS, Springer Verlag, 178 (1992), 272-284.

W. LitTMAn AND B. Liv, On the spectral properties and stabilization of acoustic flow, SIAM J. Appl. Math. 1 (1998), 17-34.

W. LitTman and M. A. HoRn, Boundary control of a Schrödinger equation with nonconstant principal part, Lecture Notes in Pure and Applied Mathematics, Marcel Dekker, 174 (1996), 101-106.

W. LitTMAN AND S.W. TAYLOR, Smoothing evolution equations and boundary control theory, J. d'Analyse Math. 59 (1992), 201-215.

E. Machtyngier, Controlabilite exacte et stabilizativ frontiere de l'equation de Schrödinger, C. R. Acad. Sci. Paris, Ser. I, Math 310 (1990), 801-806.

Mizohata, personal communication.

J. Puel and M. Tucsnak, Boundary stabilization for the von Karman equations, SIAM J. Control 33 (1996), 255-273.

J. RALSTON, Gaussian beams and the propagation of singularities, Studies in Partial Differential Equations, W. Littman (ed.), MAA Studies in Mathematics 23 (1982), 206-248.

D.L. RusselL, Controllability and stabilizability theory for linear partial differential equations, recent progress and open questions, SIAM REVIEW 20 (1978), 639-739.

T. Shirota, A remark on the unique continuation theorem for certain fourth order elliptic equations, Proc. Japan Acad. 36 (1960), $571-573$.

R. Schoen and S.-T. Yau, Lectures on Differential Geometry, International Press, Boston 1994.

D. TATARU, A-priori pseudoconvexity energy estimates in domains with boundary applications to exact boundary controllability for conservative PDEs, Ph.D. Thesis, University of Virginia, May 1992.

D. TAtaru, Boundary controllability for conservative PDEs, Appl. Math. \& Optimiz. 31 (1995), 257-295.

D. Tataru, A-priori estimates of Carleman's type in domains with boundaries, J. Math. Pures et Appl. 73 (1994), 355-387.

D. Tataru, Unique continuation for solutions to PDEs, between Hörmander's theorem and Holmgren's theorem, Comm. Partial Diff. Eqns. 20(5 \& 6) (1995), 855-884.

D. TATARu, On the regularity of boundary traces for the wave equation, Ann. Scuola Norm. Sup. Pisa Cl. Sci. 26(4) (1998), 185-206. 
D. TATARU, Unique continuation for partially differential operators with partial analytic coefficient, J. Math. Pure Appl. 78 (1999), 505-521.

D. Tataru, Carleman estimates and unique continuation for solutions to boundary value problems, J. Math. Pure Appl. 75 (1996), $367-408$.

D. TATARU, Unique continuation problems for PDEs, this volume.

[Tay.1]

A.E. TAYlor And D.C. LAY, Introduction to Functional Analysis, 2nd ed., John Wiley, New York, 1980.

M. TAYlor, Partial Differential Equations, Vols. 1, 2, Springer-Verlag, 1991.

M. TAYLOR, Tools for P.D.E.: Pseudodifferential Operators, Paradifferential Operators, and Layer Potentials.

R. Triggiani, Exact controllability on $L_{2}(\Omega) \times H^{-1}(\Omega)$ of the wave equation with Dirichlet B.C., $A M O$ (1988), 241-247.

R. Triggiani, Carleman estimates and exact boundary controllability for a system of coupled non-conservative Schrödinger equations, Rend. Istit. Math. Univ. Trieste (Italy), suppl. Vol. XXVIII (1997), 453-504; volume in memory of P. Grisvard.

R. Triggiani, Regularity theory, exact controllability and optimal quadratic cost problem for spherical shells with physical boundary controls. Invited paper in special issue for Control and Cybernetics, Polish Academy of Sciences, Control Problems for Partial Differential Equations 25(3) (1996), 553-569.

Schrödinger equations with variable coefficients, Control \& Cybernetics 28 (1999), 627-664. Special volume on control of PDEs.

[T-Y.2] R. TRiggiani AND P.F. YAO, Carleman estimates with no lower-order terms for general Riemannian wave equations. Global uniqueness and observability in one shot, Appl. Math. 86 Optim. 46 (Sept./Dec. 2002), 331-375. Special issue dedicated to J.L. Lions.

[Wu.1] H. Wu, The Bochner technique in differential geometry, Math. Reports 3 (Part 2), Hardwood Academic Publishers, London, Paris, 1988.

[Y.1] P.F. YAO, On the observability inequalities for exact controllability of wave equations with variable coefficients, SIAM J. Control Optim. 37 (1999), 1568-1599.

[Y.2] P.F. YAO, Observability inequalities for the Euler-Bernoulli plate with variable coefficients, Contemporary Mathematics, Amer. Math. Soc. 268 (2000), 383-406.

[Y.3] P.F. YAO, On the shallow shell equations, manuscript, 1999.

[Y.4] P.F. YAO, Observability inequalities for shallow shells, SIAM J. Control 38 (2000), 1729-1756.

[Z.1] E. ZuAZuA, Exact boundary controllability for the semilinear wave equation, in Non-Linear PDEs and their Applications, Research Notes in Mathematics, Pitman, Boston (19), 1265-12. 